\title{
Stacks of uniform cyclic covers of curves and their Picard groups
}

\author{
Flavia Poma, Mattia Talpo and Fabio Tonini
}

\begin{abstract}
We study the stack $\mathcal{B}_{h, g, n}$ of uniform cyclic covers of degree $n$ between smooth curves of genus $h$ and $g$ and, for $h \gg g$, present it as an open substack of a vector bundle over the universal Jacobian stack of $\mathcal{M}_{g}$. We use this description to compute the integral Picard group of $\mathcal{B}_{h, g, n}$, showing that it is generated by tautological classes of $\mathcal{B}_{h, g, n}$.
\end{abstract}

\section{Introduction}

Let $k$ be a field and let $h, g, n$ be nonnegative integers with $n \geqslant 2$. We denote by $\mathcal{B}_{h, g, n}$ the stack over $k$ of triples $(D \longrightarrow S, C \longrightarrow S, f)$ where $D \longrightarrow S$ is a smooth, geometrically connected genus $h$ curve, $C \longrightarrow S$ is a smooth, geometrically connected genus $g$ curve and $f: D \longrightarrow C$ is a uniform cyclic cover of degree $n$ (see Section 4 for a definition of uniform cyclic covers). The aim of this paper is to describe the structure of $\mathcal{B}_{h, g, n}$ and compute its integral Picard group.

This work was inspired by the results in [AV04] and [BV12], where the authors compute the Picard group of similar moduli problems, namely the stacks of uniform cyclic covers of projective spaces and of triple covers of curves of genus zero, respectively. However, the methods used here are different, as we do not use a presentation of $\mathcal{B}_{h, g, n}$ as a quotient stack. Another source of inspiration and, in fact, the starting point of our computation in genus one was the classical result of Mumford about the Picard group of the stack $\mathcal{M}_{1,1}$ of elliptic curves (see [Mum63] and also [FO10]).

This paper was born as a study of double covers of genus one curves, that is, of the stacks $\mathcal{B}_{h, 1,2}$ (which also explains the use of the letter $\mathcal{B}$, which stands for 'bielliptic'). The main obstacle in generalizing the results for $g \geqslant 2$ was the computation of the Picard group of the universal Jacobian of $\mathcal{M}_{g}$ (see below for a definition), since the methods we used for the same problem in genus one fail in higher genera. This last problem was solved in [MV14], allowing the generalization for higher genera.

Let us also remark that the case $n=2$ is the most interesting from a "geometric" point of view, since (in characteristic different from two) all covers of degree two are uniform cyclic and, therefore, $\mathcal{B}_{h, g, 2}$ is the stack of double covers between smooth curves of genera $h$ and $g$.

Received 9 Aprils 2014, accepted in final form 22 September 2014.

2010 Mathematics Subject Classification 14D23 (primary), 14C22, $14 \mathrm{H} 52$ (secondary).

Keywords: cyclic cover, Picard group, curve, moduli

This journal is (C) Foundation Compositio Mathematica 2015. This article is distributed with Open Access under the terms of the Creative Commons Attribution Non-Commercial License, which permits non-commercial reuse, distribution, and reproduction in any medium, provided that the original work is properly cited. For commercial re-use, please contact the Foundation Compositio Mathematica.

The second author was supported by the Max Planck Institute for Mathematics of Bonn. The third author was supported by the project SFB 647: Space-Time-Matter. Analytic and Geometric Structures. 


\section{Flavia Poma, Mattia Talpo and Fabio Tonini}

The Picard group of $\mathcal{B}_{h, 0, n}$ was already computed in [AV04, Theorem 5.1]. Here the authors introduce moduli stacks of uniform cyclic covers of projective spaces, denoted by $\mathcal{H}_{\mathrm{sm}}(r, n, d)$ for $r, n, d>0$. In the one-dimensional case $r=1$ we have $\mathcal{H}_{\mathrm{sm}}(1, n, d)=\mathcal{B}_{h, 0, n}$, where $d, n, h$ are related by the expression (1) below. In this paper we provide an alternative method for the computation of Pic $\mathcal{B}_{h, 0, n}$ which extends to higher genera.

In [Pag13] the author introduces moduli stacks of abelian covers of curves, which are related to our stacks $\mathcal{B}_{h, g, n}$ in the cyclic, totally ramified case. Let $\mathcal{Y}_{g, r, n}$ be the stack of tuples $\left(D, C, f, \sigma_{1}, \ldots, \sigma_{r}\right)$ where $\left(C, \sigma_{1}, \ldots, \sigma_{r}\right)$ is an $r$-pointed curve of genus $g$ and $f: D \longrightarrow C$ is a uniform cyclic cover of degree $n$ whose ramification locus is the union of the sections $\sigma_{1}, \ldots, \sigma_{r}$. By forgetting the sections we obtain a functor $\mathcal{Y}_{g, n d, n} \longrightarrow \mathcal{B}_{h, g, n}$, where $d, g, h, n$ are related by the expression (1) below, which is an $S_{n d}$-torsor. In [Pag13, Theorem 3] is proved that $\mathcal{Y}_{g, n d, n}$, which is denoted by $\mathcal{M}_{1, n d}(\mathrm{~B}(\mathbb{Z} / n \mathbb{Z}),(1), \ldots,(1))$, has trivial rational Picard group for $n d>0$, which also implies the vanishing of the rational Picard group of $\mathcal{B}_{h, 1, n}$. In this paper we recover this last result by explicitly describing the integral Picard group of $\mathcal{B}_{h, 1, n}$, but we cannot directly deduce the result in [Pag13, Theorem 3].

The main result of this paper is the following.

Theorem A. Let $h, g, n$ be nonnegative integers with $n \geqslant 2$ and set

$$
d=2 \frac{h+n(1-g)-1}{n(n-1)}, \text { so that } h=1+n(g-1)+\frac{n(n-1)}{2} d .
$$

The stack $\mathcal{B}_{h, g, n}$ is not empty if and only if $d \in \mathbb{N}$. Assume $d \in \mathbb{N}$. The stack $\mathcal{B}_{h, g, n}$ is algebraic and of finite type over $k$ and, if $n d>2 g-2$ or char $k \nmid n$, the forgetful functor $\mathcal{B}_{h, g, n} \longrightarrow \mathcal{M}_{g}$ is smooth and surjective.

Let $\pi: \mathcal{C} \longrightarrow \mathcal{B}_{h, g, n}$ be the universal genus $g$ curve, let $f: \mathcal{D} \longrightarrow \mathcal{C}$ be the universal uniform cyclic cover of degree $n$ and let $\mathcal{L}$ be the dual of the degree one part of the $\mu_{n}$-equivariant sheaf $f_{*} \mathcal{O}_{\mathcal{D}}$. The sheaf $\mathcal{L}$ is invertible of degree $d$ over $\mathcal{C}$ and we have the following.

(i) If $g=0$, we have

$$
\text { Pic } \mathcal{B}_{h, 0, n} \simeq \begin{cases}\mathbb{Z} / 2 n(n d-1) \mathbb{Z} & \text { generated by } \pi_{*}\left(\mathcal{L} \otimes \omega_{\pi}^{d / 2}\right) \text { if } d \text { is even, } \\ \mathbb{Z} / n(n d-1) \mathbb{Z} & \text { generated by } \operatorname{det} \pi_{*}\left(\mathcal{L} \otimes \omega_{\pi}^{(d-1) / 2}\right) \text { if } d \text { is odd }\end{cases}
$$

(ii) If $g=1$, then Pic $\mathcal{B}_{h, 1, n}$ is generated by $\pi_{*} \omega_{\pi}$ and $\operatorname{det} \pi_{*} \mathcal{L}$ with relations

$$
\begin{array}{ll}
\left(\pi_{*} \omega_{\pi}\right)^{4} \text { and } \operatorname{det} \pi_{*} \mathcal{L} & \text { if } h=1, n=2, \operatorname{char} k \nmid 6, \\
\left(\pi_{*} \omega_{\pi}\right)^{6} \text { and }\left(\pi_{*} \mathcal{L}\right)^{2} \otimes\left(\pi_{*} \omega_{\pi}\right)^{-2} & \text { if } h=n=2, \operatorname{char} k \neq 2, \\
\left(\pi_{*} \omega_{\pi}\right)^{12} \text { and }\left(\operatorname{det} \pi_{*} \mathcal{L}\right)^{2 n^{2}} \otimes\left(\pi_{*} \omega_{\pi}\right)^{n(d n+d-2 n)} & \text { if } n d>2, \operatorname{char} k \nmid n d .
\end{array}
$$

As an abstract group, we have

$$
\text { Pic } \mathcal{B}_{h, 1, n} \simeq \begin{cases}\mathbb{Z} / 4 \mathbb{Z} & \text { if } h=1, n=2, \text { char } k \nmid 3, \\ \mathbb{Z} / 3 \mathbb{Z} \times \mathbb{Z} / 2 \mathbb{Z} \times \mathbb{Z} / 2 \mathbb{Z} & \text { if } h=n=2, \\ \mathbb{Z} / 3 \mathbb{Z} \times \mathbb{Z} / 4 \mathbb{Z} \times \mathbb{Z} / 2 n^{2} \mathbb{Z} & \text { if } n d>2, \frac{n(d n+d-2 n)}{2} \text { is even, char } k \nmid n d, \\ \mathbb{Z} / 3 \mathbb{Z} \times \mathbb{Z} / 2 \mathbb{Z} \times \mathbb{Z} / 4 n^{2} \mathbb{Z} & \text { if } n d>2, \frac{n(d n+d-2 n)}{2} \text { is odd, char } k \nmid n d .\end{cases}
$$

(iii) Assume that $k$ is algebraically closed of characteristic zero and $n d>2 g-2$ and $g \geqslant 4$, or $n d>2 g-1$ and $g \geqslant 3$, or $n d>2 g$ and $g \geqslant 2$. Then Pic $\mathcal{B}_{h, g, n}$ is generated by $\operatorname{det} \pi_{*} \omega_{\pi}$, 
$d_{\pi}(\mathcal{L})$ and $\operatorname{det} \pi_{*}\left(\mathcal{L} \otimes \omega_{\pi}\right)$ (see Remark 2.4 for a definition of $\left.d_{\pi}(-)\right)$ with the only relation

$$
\left(\operatorname{det} \pi_{*} \omega_{\pi}\right)^{-2 n^{2}} \otimes d_{\pi}(\mathcal{L})^{n(n-1)} \otimes\left(\operatorname{det} \pi_{*}\left(\mathcal{L} \otimes \omega_{\pi}\right)\right)^{n(n+1)}
$$

except for the case $g=2$, for which we need to add the relation $\left(\operatorname{det} \pi_{*} \omega_{\pi}\right)^{10}$. As an abstract group, we have

$$
\operatorname{Pic} \mathcal{B}_{h, g, n} \simeq \begin{cases}\mathbb{Z} / 2 n \mathbb{Z} \times \mathbb{Z} / 10 \mathbb{Z} \times \mathbb{Z} & \text { if } g=2 \text { and } n \text { is odd, } \\ \mathbb{Z} / n \mathbb{Z} \times \mathbb{Z} / 10 \mathbb{Z} \times \mathbb{Z} & \text { if } g=2 \text { and } n \text { is even, } \\ \mathbb{Z} / 2 n \mathbb{Z} \times \mathbb{Z}^{2} & \text { if } g>2 \text { and } n \text { is odd, } \\ \mathbb{Z} / n \mathbb{Z} \times \mathbb{Z}^{2} & \text { if } g>2 \text { and } n \text { is even. }\end{cases}
$$

When $h=n(g-1)+1$ (in particular $g \geqslant 1$ ), that is $d=0$, uniform cyclic covers of degree $n$ become $\mu_{n}$-torsors. This case is not covered by the theorem above, except for $h=g=1$ and $n=2$, where Pic $\mathcal{B}_{1,1,2}$ is computed by an ad hoc variation of the methods used in higher genera and degrees. When $d>0$, uniform cyclic covers are never étale and the stacks $\mathcal{B}_{h, g, n}$ share a common description that we are now going to explain.

Fix $g \geqslant 0, d, n>0$ and assume char $k \nmid n$ or $g=0$ and, if $g=1$, char $k \nmid d$. Set $\mathcal{U}_{d, g, n}$ for the stack of triples $(C \longrightarrow S, \mathcal{Q}, s)$ where $C$ is a smooth, geometrically connected curve of genus $g$ and $\mathcal{Q}$ is a degree $d$ invertible sheaf with a section $s \in \mathcal{Q}^{n}$ that is not identically zero on any of the geometric fibers of $C \longrightarrow S$. The forgetful map $\mathcal{U}_{d, g, n} \longrightarrow \mathcal{M}_{g}$ defines the (universal) genus $g$ curve $\pi: \mathcal{C} \longrightarrow \mathcal{U}_{d, g, n}$ together with an invertible sheaf $\mathcal{L}$ of degree $d$ on $\mathcal{C}$ and a section $t \in \mathcal{L}^{n}$. The zero locus $\mathcal{H}_{d, g, n}$ of $t$ in $\mathcal{C}$ is a degree $n d$ cover of $\mathcal{U}_{d, g, n}$ and $\mathcal{B}_{h, g, n}$, where $d, h, g, n$ are related by the expression (1) above, can be identified with the étale locus of $\mathcal{H}_{d, g, n} \longrightarrow \mathcal{U}_{d, g, n}$ inside $\mathcal{U}_{d, g, n}$ (see Proposition 4.4). Since $\mathcal{U}_{d, g, n}$ is smooth and algebraic, the problem of computing Pic $\mathcal{B}_{h, g, n}$ splits in two parts: compute Pic $\mathcal{U}_{d, g, n}$ and describe the complement of $\mathcal{B}_{h, g, n}$ in $\mathcal{U}_{d, g, n}$.

Denote by $\mathcal{J} a c_{d, g}$ the stack of pairs $(C \longrightarrow S, \mathcal{Q})$ where $C$ is a smooth, geometrically connected genus $g$ curve and $\mathcal{Q}$ is a degree $d$ invertible sheaf on $C$. This is the so-called universal Jacobian of degree $d$ on $\mathcal{M}_{g}$. Assume $n d>2 g-2$. The forgetful functor $\mathcal{U}_{d, g, n} \longrightarrow \mathcal{J} a c_{d, g}$ makes $\mathcal{U}_{d, g, n}$ into the complement of the zero section of a vector bundle of rank $n d+1-g$ over $\mathcal{J}_{a c_{d, g}}$ (this description is no longer true in general when $n d \leqslant 2 g-2$ and this is why Theorem A does not cover this case). When $n \geqslant 2$ and since $n d+1-g \geqslant 2$, we can conclude that Pic $\mathcal{U}_{d, g, n} \simeq \operatorname{Pic} \mathcal{J} a c_{d, g}$. When $g \geqslant 2$, the group Pic $\mathcal{J} a c_{d, g}$ has been computed in [MV14]. If $g=0$, then Pic $\mathcal{J} a c_{d, 0} \simeq \mathbb{Z}$ (see Proposition 2.6). The case $g=1$ is harder than the case $g=0$ and our treatment differs from the methods used in [MV14] for $g \geqslant 2$. The result is that, if $g=1$, then Pic $\mathcal{J} a c_{d, 1} \simeq \mathbb{Z} / 12 \times \mathbb{Z}$ (see Theorem 2.9) and it has been obtained by proving that the functor $\mathcal{J} a c_{d, 1} \longrightarrow \mathcal{M}_{1,1}$ that maps $(E, \mathcal{Q})$ to $\left(\underline{\operatorname{Pic}}_{E}^{0},\left[\mathcal{O}_{E}\right]\right)$ is a trivial gerbe, that is, $\mathcal{J} a c_{d, 1} \simeq \mathrm{B}_{\mathcal{M}_{1,1}} G_{d}$ over $\mathcal{M}_{1,1}$, where $G_{d}$ is a smooth affine group over $\mathcal{M}_{1,1}$, and by computing the group of characters of $G_{d}$. The geometric fibers of $G_{d} \longrightarrow \mathcal{M}_{1,1}$ are particular cases of Theta groups, first defined by Mumford in his paper [Mum66] (see Proposition 2.16).

The last part in the computation of $\operatorname{Pic} \mathcal{B}_{h, g, n}$ is the description of $\mathcal{U}_{d, g, n}-\mathcal{B}_{h, g, n}$. Topologically this closed substack coincides with the discriminant locus $\mathcal{Z}_{d, g, n}$ of the cover $\mathcal{H}_{d, g, n} \longrightarrow \mathcal{U}_{d, g, n}$. By standard theory of covers, the locus $\mathcal{Z}_{d, g, n}$ can be described as the zero locus of a section (the discriminant section) of an invertible sheaf of $\mathcal{U}_{d, g, n}$. The key point for the computation of Pic $\mathcal{B}_{h, g, n}$ is that $\mathcal{Z}_{d, g, n}$ is reduced and, if $n d>2 g$ or $n d>2 g-1$ and $g \geqslant 3$ or $n d>2 g-2$ and $g \geqslant 4$, irreducible (see Theorem 3.2). In the special case $g=1, h=n=2$ (so that $d=1$ and $n d=2 g$ ), ad hoc methods show that $\mathcal{Z}_{1,1,2}$ is a disjoint union of two irreducible components, allowing the computation of Pic $\mathcal{B}_{2,1,2}$ (see Theorem 3.2). It is not clear whether 


\section{Flavia Poma, Mattia Talpo and Fabio Tonini}

$\mathcal{Z}_{d, g, n}$ is irreducible for the remaining values of $h, g, n$. The geometry of the loci $\mathcal{Z}_{d, g, n}$ is studied by reducing to the case $n=1$, showing that $\mathcal{U}_{d, g, 1} \simeq \operatorname{Hilb}_{\mathcal{M}_{g, 1} / \mathcal{M}_{g}}$ (see the section Notation) and that $\mathcal{H}_{d, g, 1} \longrightarrow \mathrm{Hilb}_{\mathcal{M}_{g, 1} / \mathcal{M}_{g}}$ is the universal cover.

We remark that Theorem $\mathrm{A}$ is obtained by expressing Pic $\mathcal{B}_{h, g, n}$ as quotient of Pic $\mathcal{J} a c_{d, g}$ by a given relation and this description holds more generally than in the hypothesis of Theorem $\mathrm{A}$ (see Proposition 4.6 for a precise statement). For instance, if $g \geqslant 2$ and char $k \nmid n$ the knowledge of the integral or rational Picard group of $\mathcal{J} a c_{d, g}$ implies the knowledge of the integral or rational Picard group of $\mathcal{B}_{h, g, n}$, respectively. Unfortunately, if $g \geqslant 2$, both the integral and rational Picard groups of $\mathcal{J} a c_{d, g}$ are known only in characteristic zero, although it seems reasonable to expect the same description for all but finitely many characteristics. See [MV14, Remark 1.4] for a discussion on the subject.

The paper is organized as follows. In Section 1 we collect useful remarks and lemmas, while in Section 2 we study the Picard group of the universal Jacobian $\mathcal{J} a c_{d, g}$ over $\mathcal{M}_{g}$ for $g=0$ and $g=1$ and explain the results for $g \geqslant 2$ obtained in [MV14]. In Section 3 we introduce the canonical covers $\mathcal{H}_{d, g, n} \longrightarrow \mathcal{U}_{d, g, n}$ and describe their discriminant loci, while in Section 4 we introduce the stacks $\mathcal{B}_{h, g, n}$ and compute their Picard groups.

\section{Notation}

Given a base scheme $S$, by the words "scheme" or "stack" we always mean scheme or stack defined over this base scheme. Moreover, by stacks we always mean a category fibered in groupoids which is a stack for the fppf (faithfully flat of finite presentation) topology. Let $\mathcal{S}$ be a stack.

A geometric point of $\mathcal{S}$ is a map $\operatorname{Spec} k \longrightarrow \mathcal{S}$, where $k$ is an algebraically closed field.

Given a sheaf of groups $G:(\mathrm{Sch} / \mathcal{S})^{\text {op }} \longrightarrow($ grps $)$, we denote by $G^{\vee}=\operatorname{Hom}_{\mathcal{S}}\left(G, \mathbb{G}_{m}\right)$ the group of characters of $G$ over $\mathcal{S}$, that is, group homomorphisms $G \longrightarrow \mathbb{G}_{m}$.

By a cover we mean an affine map $\mathcal{X} \stackrel{f}{\longrightarrow} \mathcal{S}$ such that $f_{*} \mathcal{O}_{\mathcal{X}}$ is locally free of finite rank. Alternatively, a cover is a finite, flat and finitely presented map $\mathcal{X} \stackrel{f}{\longrightarrow} \mathcal{S}$. The degree of $f$ is the rank of $f_{*} \mathcal{O}_{\mathcal{X}}$. The discriminant section $s_{f} \in \operatorname{det}\left(f_{*} \mathcal{O}_{\mathcal{X}}\right)^{-2}$ of $f$ is the determinant of the map

$$
f_{*} \mathcal{O}_{\mathcal{X}} \longrightarrow f_{*} \mathcal{O}_{\mathcal{X}} \vee x \longmapsto \operatorname{tr}_{f_{*}} \mathcal{O}_{\mathcal{X}}(x \cdot-),
$$

where $\operatorname{tr}$ denotes the trace map. The discriminant locus of $f$ is the zero locus in $\mathcal{S}$ of $s_{f}$. The discriminant section is stable under base change and the complement of the discriminant locus of $f$ is the étale locus of $f$ in $\mathcal{S}$.

A genus $g$ curve over $\mathcal{S}$ is a representable (by algebraic spaces), proper and smooth map $\mathcal{C} \longrightarrow \mathcal{S}$ of stacks whose geometric fibers are connected genus $g$ curves. Let $\pi: \mathcal{C} \longrightarrow \mathcal{S}$ be a genus $g$ curve. We say that an invertible sheaf $\mathcal{L}$ on $\mathcal{C}$ has degree $d \in \mathbb{Z}$ if the pull-back of $\mathcal{L}$ on every geometric fiber of $\pi: \mathcal{C} \longrightarrow \mathcal{S}$ has degree $d$. We denote by $\omega_{\pi}$ the relative dualizing sheaf, which is an invertible sheaf on $\mathcal{C}$ of degree $2 g-2$.

We denote by $\mathcal{M}_{g}$ the stack of genus $g$ curves and by $\mathcal{M}_{g, 1}$ the stack of genus $g$ curves with a section. The forgetful functor $\mathcal{M}_{g, 1} \longrightarrow \mathcal{M}_{g}$ is a genus $g$ curve, called the universal genus $g$ curve of $\mathcal{M}_{g}$. Given a map $\mathcal{S} \longrightarrow \mathcal{M}_{g}$, the universal genus $g$ curve of $\mathcal{S}$ is the base change $\mathcal{S} \times \mathcal{M}_{g} \mathcal{M}_{g, 1} \longrightarrow \mathcal{S}$

Let $\mathcal{X}$ be another stack and let $f: \mathcal{X} \longrightarrow \mathcal{S}$ be a representable map. We denote by Hilb ${ }_{\mathcal{X} / \mathcal{S}}^{n}$ (or simply Hilb ${ }_{\mathcal{X}}^{n}$ whenever $\mathcal{S}$ is clear from the context) the stack over $\mathcal{S}$ whose objects over $S \longrightarrow \mathcal{S}$ are closed subschemes $Z \subseteq \mathcal{X} \times \mathcal{S} S$ such that the projection $Z \longrightarrow S$ is a degree $n$ cover. When $f: \mathcal{X} \longrightarrow \mathcal{S}$ is a projective map of schemes, $\operatorname{Hilb}_{\mathcal{X} / \mathcal{S}}^{n}$ is the usual Hilbert scheme of points. 


\section{StACKS OF UNIFORM CYCLIC COVERS OF CURVES AND THEIR PICARD GROUPS}

We denote by $\underline{\operatorname{Pic}} \mathcal{X} / \mathcal{S}$ (or simply $\underline{\mathrm{Pic}}_{\mathcal{X}}$ whenever $\mathcal{S}$ is clear from the context) the stack over $\mathcal{S}$ which is the fppf sheafification of the functor $(\mathrm{Sch} / \mathcal{S})^{\mathrm{op}} \longrightarrow$ (sets) that maps $S \longrightarrow \mathcal{S}$ to the set $\operatorname{Pic}(\mathcal{X} \times \mathcal{S} S)$. If $f: \mathcal{X} \longrightarrow \mathcal{S}$ is a curve, we also denote by $\underline{\operatorname{Pic}}_{\mathcal{X} / \mathcal{S}}^{d}$ (or simply $\underline{\operatorname{Pic}}_{\mathcal{X}}^{d}$ ) the substack of $\underline{\mathrm{Pic}} \mathcal{X} / \mathcal{S}$ of classes that are locally given by invertible sheaves having degree $d$ on the geometric fibers of $f$. Given $n \in \mathbb{Z}$ we will denote by $[n]: \underline{\operatorname{Pic}}_{\mathcal{X} / \mathcal{S}} \longrightarrow \underline{\operatorname{Pic}}_{\mathcal{X} / \mathcal{S}}\left(\right.$ or $[n]: \underline{\operatorname{Pic}}_{\mathcal{X} / \mathcal{S}}^{d} \longrightarrow \underline{\operatorname{Pic}}_{\mathcal{X} / \mathcal{S}}^{d n}$ if $\mathcal{X}$ is a curve over $\mathcal{S}$ ) the map induced by the multiplication by $n$.

The formation of $\operatorname{Hilb}_{\mathcal{X} / \mathcal{S}}^{n}, \underline{\operatorname{Pic}}_{\mathcal{X} / \mathcal{S}}$ and $\underline{\operatorname{Pic}}_{\mathcal{X} / \mathcal{S}}^{d}$ commute with arbitrary base change of the base $\mathcal{S}$. In particular, if $\mathcal{X} \longrightarrow \mathcal{S}$ is a curve, the stacks $\operatorname{Hilb}_{\mathcal{X} / \mathcal{S}}^{d}$ and $\underline{\operatorname{Pic}}_{\mathcal{X} / \mathcal{S}}^{d}$ for $d \in \mathbb{Z}$ are smooth over $\mathcal{S}$.

If $\mathcal{X}$ is an algebraic stack, we will denote by $|\mathcal{X}|$ the topological space associated with $\mathcal{X}$.

Almost every stack $\mathcal{X}$ that we will introduce has a given map to $\mathcal{M}_{g}$, for some given $g$, and therefore has a given genus $g$ curve over it, that, as remarked above, we will call the universal curve over (or of) $\mathcal{X}$. With abuse of notation we will usually denote the curves by the same symbol $\mathcal{C}$ for the total space and $\pi$ for the structure map, that is, $\pi: \mathcal{C} \longrightarrow \mathcal{X}$, but with the convention that this notation is fixed and remains coherent inside the statement of a lemma, proposition, theorem ... and its proof. The use of different symbols for such curves seemed to us not practical, while the use of subscripts would have encumbered the notation too much. Moreover, this notation is supported by the idea that genus $g$ curves can be seen as restriction of the universal curve over $\mathcal{M}_{g}$. Indeed, if $\pi: \mathcal{C} \longrightarrow \mathcal{M}_{g}$ is the universal curve and $q: \mathcal{X} \longrightarrow \mathcal{M}_{g}$ is a map, $\mathcal{C}$ can be seen as the functor $F:\left(\mathrm{Sch} / \mathcal{M}_{g}\right)^{\text {op }} \longrightarrow$ (sets) which maps a genus $g$ curve $C$ over a scheme $S$ to the set of sections $C(S)$, while $q$ corresponds to a map $\mathrm{Sch} / \mathcal{X} \longrightarrow \mathrm{Sch} / \mathcal{M}_{g}$. The universal curve $\mathcal{C} \times{ }_{\mathcal{M}_{g}} \mathcal{X} \longrightarrow \mathcal{X}$ of $\mathcal{X}$ then corresponds to the restriction of the functor $F$ along the map $\mathrm{Sch} / \mathcal{X} \longrightarrow \mathrm{Sch} / \mathcal{M}_{g}$

\section{Preliminaries}

In this section we collect some general results and remarks that will be useful in the next sections. These results are well known, but for some of them we have decided to include a proof for completeness and because of the lack of exhaustive references. In this section we consider Spec $\mathbb{Z}$ as the base scheme.

Proposition 1.1. Let $f: \mathcal{X} \longrightarrow \mathcal{Y}$ be a locally finitely presented map of algebraic stacks and let $\alpha: \mathcal{F} \longrightarrow \mathcal{H}$ be a map of finitely presented quasi-coherent sheaves on $\mathcal{X}$. If $\mathcal{H}$ is flat over $\mathcal{Y}$, then $\alpha$ is injective on the geometric fibers of $f$ if and only if $\alpha$ is injective and Coker $\alpha$ is flat over $\mathcal{Y}$. In this case $\alpha$ remains injective after any base change $\mathcal{Y}^{\prime} \longrightarrow \mathcal{Y}$ from an algebraic stack.

Proof. It is easy to see that taking atlases of $\mathcal{Y}$ and $\mathcal{X}$, we can reduce the problem to the case of schemes, where it follows from [Gro66, Proposition 11.3.7].

REMARK 1.2. Let $\mathcal{Y}$ be an algebraic stack and let $G$ be a sheaf of groups over $\mathcal{Y}$. Then we have a natural isomorphism

$$
\operatorname{Pic} \mathrm{B}_{\mathcal{Y}} G \simeq \operatorname{Pic} \mathcal{Y} \oplus \operatorname{Hom}\left(G, \mathbb{G}_{m}\right) .
$$

Indeed, by descent, an invertible sheaf over $\mathrm{B} \mathcal{Y} G$ is given by a pair $(\mathcal{L}, \rho)$ where $\mathcal{L}$ is an invertible sheaf over $\mathcal{Y}$ and $\rho \in \operatorname{Hom}\left(G, \mathbb{G}_{m}\right)$, which defines an action of $G$ on $\mathcal{L}$ given by $\rho: G \longrightarrow \mathbb{G}_{m} \simeq$ $\underline{\operatorname{Aut}}(\mathcal{L})$.

In some proofs we will use dimension counting for algebraic stacks. We recall here some 


\section{Flavia Poma, Mattia Talpo and Fabio Tonini}

properties which are well known for schemes. We refer to [MBL99, Chapter 11] for definitions and basics about dimension theory for stacks.

REMARK 1.3. Let $f: \mathcal{X} \longrightarrow \mathcal{Y}$ be a locally of finite type map of algebraic stacks. Let $\xi \in|\mathcal{X}|$. If $\eta:$ Spec $k \longrightarrow \mathcal{Y}$, where $k$ is a field, maps to $f(\xi)$ and $x \in|\mathcal{X} \times \mathcal{Y} k|$ maps to $\xi$ via the projection, then the number

$$
\operatorname{dim}_{\xi} f=\operatorname{dim}_{x}(\mathcal{X} \times \mathcal{Y} k) \in \mathbb{Z}
$$

does not depend on $\eta$ and $x$. Indeed, by standard arguments about fiber products one can reduce to the case $\mathcal{Y}=$ Spec $k$ and show that if $L / k$ is a field extension and $\xi^{\prime} \in\left|\mathcal{X} \times{ }_{k} L\right|$ maps to $\xi \in|\mathcal{X}|$ then $\operatorname{dim}_{\xi^{\prime}}\left(\mathcal{X} \times{ }_{k} L\right)=\operatorname{dim}_{\xi} \mathcal{X}$. Using the definition of dimension for stacks one can assume that $\mathcal{X}$ is a scheme. In this case the result is standard (see for instance [SP14, Tag 02FW]).

Definition 1.4. Given $n \in \mathbb{Z}$, a locally of finite type map $f: \mathcal{X} \longrightarrow \mathcal{Y}$ of algebraic stacks has (pure) relative dimension $n$ if all (the irreducible components of all) the fibers have dimension $n$.

REMARK 1.5. If $\mathcal{X}$ is a quasi-compact algebraic stack, there exist $n \in \mathbb{N}$ and an atlas $X \longrightarrow \mathcal{X}$ of pure relative dimension $n$, where $X$ is a quasi-compact scheme. Indeed, if $P: X^{\prime} \longrightarrow \mathcal{X}$ is an atlas from a quasi-compact scheme $X^{\prime}$, by [MBL99, Proposition 11.10] we have a decomposition $X^{\prime}=\bigsqcup_{r=0}^{n} X_{r}$ such that, if $x \in X_{r}, r=\operatorname{dim}_{x} P\left(=\operatorname{dim}_{x} P_{\mid X_{r}}\right)$. Taking into account [MBL99, Corollary 11.11] the map $Q: X=\bigsqcup_{r=0}^{n} \mathbb{A}_{X_{r}}^{n-r} \longrightarrow \mathcal{X}$ satisfies $\operatorname{dim}_{x} Q=n$ for all $x \in X$; that is, it has pure relative dimension $n$.

Proposition 1.6. Let $f: \mathcal{X} \longrightarrow \mathcal{Y}$ be a flat and locally of finite type map of locally noetherian algebraic stacks. Then

$$
\operatorname{dim}_{\xi} \mathcal{X}=\operatorname{dim}_{\xi} f+\operatorname{dim}_{f(\xi)} \mathcal{Y} \text { for all } \xi \in|\mathcal{X}|
$$

In particular, if $f$ has relative dimension $r \in \mathbb{Z}$, then

$$
\operatorname{dim} \mathcal{X}=\operatorname{dim} \mathcal{Y}+r .
$$

Moreover, if $\mathcal{Y}$ is locally of finite type over a field or $\mathbb{Z}, f$ has pure relative dimension $r \in \mathbb{Z}$ and $\mathcal{Y}^{\prime}$ is an irreducible component of $\mathcal{Y}$, then all irreducible components of $f^{-1}\left(\mathcal{Y}^{\prime}\right)$ dominate $\mathcal{Y}^{\prime}$ and have dimension $\operatorname{dim} \mathcal{Y}^{\prime}+r$.

Proof. We first prove (2) when $\mathcal{X}$ and $\mathcal{Y}$ are schemes. By [Gro66, Corollary 14.2.6] and since fibers have the subspace topology, one may assume that $f$ is surjective, of relative dimension $n \in \mathbb{N}$ and translate (2) in $\operatorname{dim} \mathcal{X}=\operatorname{dim} \mathcal{Y}+n$. By [Gro66, Theorem 14.2.1] we have $\operatorname{dim}\left(\mathcal{O}_{\mathcal{X}, x}\right)=\operatorname{dim}\left(\mathcal{O}_{f^{-1}(f(x)), x}\right)+\operatorname{dim}\left(\mathcal{O}_{\mathcal{Y}, f(x)}\right)$ for all $x \in \mathcal{X}$. Since for all $y \in \mathcal{Y}$ we have $\max _{x \in f^{-1}(y)}\left\{\operatorname{dim}\left(\mathcal{O}_{f^{-1}(y), x}\right)\right\}=n$, we get the desired expression.

When $f$ is a smooth atlas (2) follows from definition of $\operatorname{dim}_{f(\xi)} \mathcal{Y}$. We show that

$$
\text { (2) for schemes } \Longrightarrow \text { (2) for algebraic spaces } \Longrightarrow \text { (2) for stacks. }
$$

Both implications follows from the same proof. One considers smooth atlases $X \longrightarrow \mathcal{X}$ and $Y \longrightarrow \mathcal{Y}$. Choose points $y \in Y$ over $f(\xi)$ and $x \in(X \times \mathcal{Y} k(y))$ mapping to $\xi$. Writing a diagram of all possible fiber products, the proof now consists in applying (2) several times on various projections of this diagram.

If $f$ has relative dimension $r$, the expression $\operatorname{dim} \mathcal{X}=\operatorname{dim} \mathcal{Y}+r$ follows from (2) and the fact that for all $\eta \in|\mathcal{Y}|$ we have $\max _{\xi \in f^{-1}(\eta)}\left\{\operatorname{dim}_{\xi} f\right\}=r$. We now consider the last claim in the statement. We may assume that $\mathcal{Y}$ is irreducible. Let $\mathcal{X}^{\prime}$ be an irreducible component of $\mathcal{X}$ with generic point $\xi$. Notice that $\mathcal{X}^{\prime}$ contains an open substack of $\mathcal{X}$. In particular, $\operatorname{dim}_{\xi} \mathcal{X}=\operatorname{dim}_{\xi} \mathcal{X}^{\prime}$. 


\section{StACKS OF UNIFORM CYCLIC COVERS OF CURVES AND THEIR PICARD GROUPS}

Moreover, since $f$ is open, $f(\xi)=\eta$ is the generic point of $\mathcal{Y}$. By (2) we have $\operatorname{dim}_{\xi} \mathcal{X}^{\prime}=\operatorname{dim}_{\eta} \mathcal{Y}+r$. Thus it suffices to show that if $\mathcal{Z}$ is an irreducible stack locally of finite type over a field or $\mathbb{Z}$ and $\zeta$ is its generic point, then $\operatorname{dim}_{\zeta} \mathcal{Z}=\operatorname{dim} \mathcal{Z}$. First, we may assume that $\mathcal{Z}$ is quasi-compact by taking an open substack of $\mathcal{Z}$ of the same dimension. Then, by Remark 1.5 there exists an atlas $P: Z \longrightarrow \mathcal{Z}$ of pure relative dimension $r$, so that $\operatorname{dim} Z=\operatorname{dim} \mathcal{Z}+r$. On the other hand, we have seen that if $z$ is a generic point of $Z$, then $\operatorname{dim}_{z} Z=\operatorname{dim}_{\zeta} \mathcal{Z}+r$. This tells us that $\operatorname{dim}_{z} Z$ does not depend on the choice of the generic point and therefore, by [Gro66, Corollary 10.6.4, Example 10.7.1], $\operatorname{dim}_{z} Z=\operatorname{dim} \overline{\{z\}}=\operatorname{dim} Z$, which implies $\operatorname{dim} \mathcal{Z}=\operatorname{dim}_{\zeta} \mathcal{Z}$.

Corollary 1.7. Let $\mathcal{X}$ be an irreducible stack of finite type over a field and let $\mathcal{Z}$ be the zero locus of a section of an invertible sheaf on $\mathcal{X}$. If $\emptyset \neq \mathcal{Z} \subsetneq \mathcal{X}$ then all irreducible components of $\mathcal{Z}$ have dimension $\operatorname{dim} \mathcal{X}-1$.

Proof. Let $P: X \longrightarrow \mathcal{X}$ be an atlas of pure relative dimension $r$ (see Remark 1.5) and let $\mathcal{Z}^{\prime}, Z^{\prime}$ and $X^{\prime}$ be irreducible components of $\mathcal{Z}, P^{-1}\left(\mathcal{Z}^{\prime}\right)$ and $X$ such that $Z^{\prime} \subseteq X^{\prime}$. Notice that $Z^{\prime} \subsetneq X^{\prime}$, because otherwise $P\left(Z^{\prime}\right)$ contains the generic point of $\mathcal{X}$. Since $Z^{\prime}$ is an irreducible component of a section of an invertible sheaf on $X^{\prime}$, we have $\operatorname{dim} Z^{\prime}=\operatorname{dim} X^{\prime}-1$. On the other hand, since $P$ has pure relative dimension $r$, we have $\operatorname{dim} Z^{\prime}=\operatorname{dim} \mathcal{Z}^{\prime}+r$ and $\operatorname{dim} X^{\prime}=\operatorname{dim} \mathcal{X}+r$.

Corollary 1.8. Let $f: \mathcal{X} \longrightarrow \mathcal{Y}$ be a map of stacks locally of finite type over a field and assume that $\mathcal{X}$ is a Deligne-Mumford stack. Then $\operatorname{dim} \overline{f(\mathcal{X})} \leqslant \operatorname{dim} \mathcal{X}$, where $\overline{f(\mathcal{X})}$ is the reduced closed substack of $\mathcal{Y}$ whose topological space is $\overline{f(|\mathcal{X}|)}$.

Proof. When $\mathcal{X}$ and $\mathcal{Y}$ are schemes, the result is standard. We show how to reduce to this case. We may assume that $f$ is dominant, so that $\overline{f(\mathcal{X})}=\mathcal{Y}$. By taking an atlas of $\mathcal{Y}$ of pure relative dimension (see Remark 1.5), we may assume that $\mathcal{Y}$ is a scheme. Moreover, we may replace $\mathcal{X}$ by a scheme because étale atlases do not change dimension.

Proposition 1.9. Let $\mathcal{X}$ be a smooth and integral algebraic stack over a field.

- If $h: \mathcal{V} \longrightarrow \mathcal{X}$ is a vector bundle of finite rank, then $h^{*}:$ Pic $\mathcal{X} \longrightarrow$ Pic $\mathcal{V}$ is an isomorphism.

- If $\mathcal{Z}$ is a closed substack of $\mathcal{X}$ of codimension greater than two, then the restriction map $\operatorname{Pic} \mathcal{X} \longrightarrow \operatorname{Pic}(\mathcal{X}-\mathcal{Z})$ is an isomorphism.

- Given $\mathcal{L}_{1}, s_{1}, \ldots, \mathcal{L}_{r}, s_{r}$, where $\mathcal{L}_{i}$ is an invertible sheaf on $\mathcal{X}$ with a nonzero global section $s_{i}$ whose zero locus $Z\left(s_{i}\right)$ is integral, the restriction map induces an isomorphism

$$
\operatorname{Pic} \mathcal{X} /\left\langle\mathcal{L}_{1}, \ldots, \mathcal{L}_{r}\right\rangle \simeq \operatorname{Pic}\left(\mathcal{X}-\left(Z\left(s_{1}\right) \cup \cdots \cup Z\left(S_{r}\right)\right)\right) .
$$

Proof. Let $\sigma: \mathcal{X} \longrightarrow \mathcal{V}$ be the zero section. We must prove that $\operatorname{Pic} \mathcal{V} \stackrel{\sigma^{*}}{\longrightarrow} \operatorname{Pic} \mathcal{X}$ is injective. Let $\mathcal{Q}$ be an invertible sheaf on $\mathcal{V}$ in the kernel and define the sheaf $F_{\mathcal{Q}}$ on the small smooth-étale site of $\mathcal{X}$ by

$$
F_{\mathcal{Q}}(U)=\operatorname{IsO}_{h^{-1}(U)}\left(\mathcal{Q}_{\mid h^{-1}(U)}, \mathcal{O}_{h^{-1}(U)}\right) .
$$

The map $\sigma$ induces a map $F_{\mathcal{Q}} \longrightarrow \underline{\operatorname{Iso}}_{\mathcal{X}}\left(\sigma^{*} \mathcal{Q}, \mathcal{O}_{\mathcal{X}}\right)$ and it suffices to prove that it is an isomorphism. This is a local question, so that we may assume that $\mathcal{X}=\operatorname{Spec} D$ is affine and that $\mathcal{V}$ is trivial. By standard intersection theory for schemes, $\mathcal{Q}$ is trivial and therefore the previous map on the global sections is just $\left(D\left[x_{1}, \ldots, x_{n}\right]\right)^{*} \simeq D^{*}$.

Let $\mathcal{U}$ be an open substack of $\mathcal{X}$. If $\mathcal{Q}$ is an invertible sheaf on $\mathcal{U}$, then by [MBL99, Corollary 15.5] there exists a coherent sheaf $\mathcal{F}$ on $\mathcal{X}$ such that $\mathcal{F}_{\mathcal{U}} \simeq \mathcal{Q}$. Then the sheaf $\mathcal{L}=\mathcal{F}^{\vee \vee}$ is a reflexive sheaf of rank one and thus invertible and $\mathcal{L}_{\mid \mathcal{U}} \simeq \mathcal{Q}$. This shows that Pic $\mathcal{X} \longrightarrow$ Pic $\mathcal{U}$ is 


\section{Flavia Poma, Mattia Talpo and Fabio Tonini}

surjective. We now use the description of divisors given in [AV04, Proof of Lemma 5.2]. Let $\mathcal{L}$ be an invertible sheaf on $\mathcal{X}$ such that $\mathcal{L}_{\mathcal{U}} \simeq \mathcal{O}_{\mathcal{U}}$. It follows that there is a divisor $\mathcal{D}$ on $\mathcal{X}$ such that $\mathcal{L} \simeq \mathcal{O}_{\mathcal{X}}(\mathcal{D})$ and the support of $\mathcal{D}$ is in $\mathcal{X}-\mathcal{U}$. In particular, if $\mathcal{X}-\mathcal{U}$ has codimension greater than two, then $\mathcal{D}=0$. For the last point the sheaves $\mathcal{L}_{i} \simeq \mathcal{O}_{\mathcal{X}}\left(Z\left(s_{i}\right)\right)$ restrict to $\mathcal{O}_{\mathcal{U}}$ on $\mathcal{U}$ and $\mathcal{L} \simeq \mathcal{L}_{1}^{m_{1}} \otimes \mathcal{L}_{r}^{m_{r}}$ where $m_{i}$ is the multiplicity of $\mathcal{D}$ in $Z\left(s_{i}\right)$ (computed on an atlas).

Lemma 1.10. Let $\pi: \mathcal{C} \longrightarrow \mathcal{S}$ be a genus $g$ curve over an algebraic stack and let $\mathcal{F}$ be a finitely presented quasi-coherent sheaf on $\mathcal{C}$, flat over $\mathcal{S}$. Then $\mathrm{R}^{j} \pi_{*} \mathcal{F}$ is locally free and satisfies base change for all $j \in \mathbb{N}$ in the following cases.

(i) The sheaf $\mathcal{F}$ is invertible of degree zero and such that $[\mathcal{F}]=0$ in $\underline{\mathrm{Pic}}_{\mathcal{C} / \mathcal{S}}^{0}$. In this case, $\pi_{*} \mathcal{F}$ is an invertible sheaf and the canonical map

$$
\pi^{*} \pi_{*} \mathcal{F} \longrightarrow \mathcal{F}
$$

is an isomorphism.

(ii) We have $\mathcal{F}=\omega_{\pi}$. The sheaf $\pi_{*} \omega_{\pi}$ has rank $g$ and $\mathrm{R}^{1} \pi_{*} \omega_{\pi} \simeq \mathcal{O}_{\mathcal{S}}$. Moreover, if $g=1$, the map $\pi^{*} \pi_{*} \omega_{\pi} \simeq \omega_{\pi}$ is an isomorphism.

(iii) The sheaf $\mathcal{F}$ is invertible of degree $d>2 g-2$ or $d<0$. In this case, $\operatorname{rk} \pi_{*} \mathcal{F}=\max \{d+1-g, 0\}$ and $\operatorname{rkR}^{1} \pi_{*} \mathcal{F}=\max \{-d-1+g, 0\}$.

(iv) The sheaf $\mathcal{F}$ is supported on a closed substack of $\mathcal{C}$ which is quasi-finite over $\mathcal{S}$. In this case, $\mathrm{R}^{1} \pi_{*} \mathcal{F}=0$.

In all of the cases above but the last one, we have an isomorphism

$$
\mathrm{R}^{1} \pi_{*} \mathcal{F} \simeq \pi_{*}\left(\mathcal{F}^{\vee} \otimes \omega_{\pi}\right)^{\vee}
$$

Proof. By [Kle80, II, Definition 10 and Theorem 21] there is a canonical map

$$
\pi_{*} \underline{\operatorname{Hom}}\left(\mathcal{F}, \omega_{\pi}\right) \longrightarrow\left(\mathrm{R}^{1} \pi_{*} \mathcal{F}\right)^{\vee}
$$

which is an isomorphism if $\mathrm{R}^{1} \pi_{*} \mathcal{F}$ satisfies base change. In this case. if $\mathcal{F}$ and $\mathrm{R}^{1} \pi_{*} \mathcal{F}$ are locally free, we get the last formula in the statement by dualizing the isomorphism above.

All the other claims follow by standard semicontinuity theorems and Riemann-Roch.

Lemma 1.11. Let $\pi: \mathcal{C} \longrightarrow \mathcal{S}$ be a genus $g$ curve over an algebraic stack and let $\mathcal{Q}$ be a degree $d$ invertible sheaf on $\mathcal{C}$ with a section $s \in \mathcal{Q}$ which is nonzero on the geometric fibers. Then the zero locus $\mathcal{Z}$ of $s$ in $\mathcal{C}$ is a degree $d$ cover of $\mathcal{S}$. When $d=1$ this defines a section $\tau: \mathcal{S} \longrightarrow \mathcal{C}$ with an isomorphism $\mathcal{O}_{\mathcal{C}}(\tau) \simeq \mathcal{Q}$ sending 1 to $s$. If in addition $g=1$, then the map $\mathcal{O}_{\mathcal{S}} \stackrel{s}{\longrightarrow} \pi_{*} \mathcal{Q}$ is an isomorphism.

Proof. By Proposition 1.1 the sequence

$$
0 \longrightarrow \mathcal{Q}^{-1} \longrightarrow \mathcal{O}_{\mathcal{C}} \longrightarrow \mathcal{O}_{\mathcal{Z}} \longrightarrow 0
$$

is universally exact over $\mathcal{S}$ and $\mathcal{Z}$ is flat over $\mathcal{S}$. Moreover, $\mathcal{Z} \longrightarrow \mathcal{S}$ is proper, finitely presented and, by looking at the geometric fibers, quasi-finite. By [Gro66, Theorem 8.11.1] we can conclude that $\mathcal{Z} \longrightarrow \mathcal{S}$ is a cover. By Riemann-Roch it has degree $d$.

Assume $d=1$. The claim about the section $\tau$ follows from standard arguments. The last claim follows from the fact that $\pi_{*} \mathcal{Q}$ is invertible and satisfies base change by Lemma 1.10 and $s \in \pi_{*} \mathcal{Q}$ is nowhere vanishing by hypothesis.

REMARK 1.12. Let $\pi: \mathcal{C} \longrightarrow \mathcal{S}$ be a genus $g$ curve over an algebraic stack and $n \in \mathbb{Z}$. The map

$[n]: \underline{\operatorname{Pic}}_{\mathcal{C} / \mathcal{S}}^{r} \longrightarrow \underline{\operatorname{Pic}}_{\mathcal{C} / \mathcal{S}}^{r n}$ is a cover of degree $n^{2 g}$, and it is étale if $n \in \mathcal{O}_{\mathcal{S}}^{*}$. Indeed, since the problem 


\section{StACKS OF UNIFORM CYCLIC COVERS OF CURVES AND THEIR PICARD GROUPS}

is local on $\mathcal{S}$, one may assume that $\mathcal{S}$ is a noetherian scheme and that $\mathcal{C} \longrightarrow \mathcal{S}$ has a section. This allows us to reduce the problem to the case $r=0$. Since $\underline{\operatorname{Pic}}_{\mathcal{C} / \mathcal{S}}^{0} \longrightarrow \mathcal{S}$ is flat and proper of relative dimension $g$, by the local flatness criterion [Gro66, Theorem 11.3.10] we may assume that $\mathcal{S}$ is the spectrum of an algebraically closed field. In this case, the result follows from [Mil08, Proposition 7.1 and Theorem 7.2].

In particular, all invertible sheaves on $\mathcal{C}$ of degree divisible by $n$ are locally for the fppf topology $\left(\right.$ on $\mathcal{S}$ ) an $n$th power of an invertible sheaf on $\mathcal{C}$. Moreover, $\left(\underline{\operatorname{Pic}}_{\mathcal{C} / \mathcal{S}}^{0}\right)[n]$ is a finite, flat and finitely presented group scheme over $\mathcal{S}$ of degree $n^{2 g}$, and it is étale if $n \in \mathcal{O}_{\mathcal{S}}^{*}$.

\section{The universal jacobian of degree $d$ over $\mathcal{M}_{g}$ and its Picard group}

In this section we assume to work over a field of characteristic $p \geqslant 0$ and we fix a nonnegative integer $g$ (the genus) and an integer $d$ (the degree).

Definition 2.1. We denote by $\mathcal{J} a c_{d, g}$ the stack of pairs $(C, \mathcal{Q})$ where $C$ is a curve of genus $g$ and $\mathcal{Q}$ is an invertible sheaf over $C$ of degree $d$. The stack $\mathcal{J} a c_{d, g}$ is called the universal Jacobian stack of degree $d$ over $\mathcal{M}_{g}$.

The aim of this section is to describe the Picard group of $\mathcal{J} a c_{d, g}$. When $g \geqslant 2$ this has already been done in [MV14]. We will deal with the remaining cases, that is, genus zero and one.

REMARK 2.2. The forgetful functor $\mathcal{J} a c_{d, g} \longrightarrow \mathcal{M}_{g}$ is the composition of a $\mathbb{G}_{m}$-gerbe $\mathcal{J} a c_{d, g} \longrightarrow$ $\underline{\mathrm{Pic}}_{\mathcal{M}_{g, 1} / \mathcal{M}_{g}}$ and the representable and smooth functor $\underline{\operatorname{Pic}}_{\mathcal{M}_{g, 1} / \mathcal{M}_{g}}^{\longrightarrow} \longrightarrow \mathcal{M}_{g}$. In particular, $\mathcal{J} a c_{d, g}$ is a smooth and integral algebraic stack.

Definition 2.3. Let $\mathcal{C} \longrightarrow \mathcal{J} a c_{d, g}$ be the universal curve over $\mathcal{J} a c_{d, g}$. By construction, there exists an invertible sheaf $\mathcal{L}$ over $\mathcal{C}$ such that

$$
\begin{aligned}
& C \stackrel{q}{\longrightarrow} \mathcal{C} \\
& \downarrow \\
& T \stackrel{(C, \mathcal{Q})}{\longrightarrow} \mathcal{J} a c_{d, g}
\end{aligned} \quad q^{*} \mathcal{L} \simeq \mathcal{Q}
$$

for all schemes $T$. We call the sheaf $\mathcal{L}$ the universal invertible sheaf over $\mathcal{C}$. Given a stack $\mathcal{Y}$ over $\mathcal{J} a c_{d, g}$ the universal invertible sheaf over the universal curve $\mathcal{C} \times{\mathcal{J} a c_{d, g}} \mathcal{Y}$ of $\mathcal{Y}$ is the pull-back of $\mathcal{L}$ via the $\operatorname{map} \mathcal{C} \times{\mathcal{J} a c_{d, g}} \mathcal{Y} \longrightarrow \mathcal{C}$.

We now describe the result in [MV14] about Pic $\mathcal{J} a c_{d, g}$ when $g \geqslant 2$.

REMARK 2.4. Let $\pi: \mathcal{C} \longrightarrow \mathcal{S}$ be a genus $g$ curve. Given an invertible sheaf $\mathcal{T}$ on $\mathcal{C}$ one can define an invertible sheaf $d_{\pi}(\mathcal{T})$ on $\mathcal{S}$, called the determinant of cohomology of $\mathcal{T}$. When $\pi_{*} \mathcal{T}$ and $\mathrm{R}^{1} \pi_{*} \mathcal{T}$ are locally free, one can simply set $d_{\pi}(\mathcal{T}) \simeq \operatorname{det} \pi_{*} \mathcal{T} \otimes\left(\operatorname{det} \mathrm{R}^{1} \pi_{*} \mathcal{T}\right)^{-1}$. We refer to [MV14] for the general definition. In this paper we just use the fact that the formation of $d_{\pi}(\mathcal{T})$ commutes with arbitrary base changes.

Notice that from Lemma 1.10 it follows that $d_{\pi}\left(\omega_{\pi}\right) \simeq \operatorname{det} \pi_{*} \omega_{\pi}$ and that, if $\mathcal{T}$ is an invertible sheaf on $\mathcal{C}$ of positive degree, then $d_{\pi}\left(\mathcal{T} \otimes \omega_{\pi}\right) \simeq \operatorname{det} \pi_{*}\left(\mathcal{T} \otimes \omega_{\pi}\right)$.

TheOrem 2.5. [MV14] Assume that the ground field is algebraically closed of characteristic 0 and that $g \geqslant 2$ and $d>0$. Let $\pi: \mathcal{C} \longrightarrow \mathcal{J} a c_{d, g}$ be the universal curve and let $\mathcal{L}$ be the universal invertible sheaf over $\mathcal{C}$. Then Pic $\mathcal{J} a c_{d, g}$ is freely generated by $\operatorname{det} \pi_{*} \omega_{\pi}, d_{\pi}(\mathcal{L})$ and $\operatorname{det} \pi_{*}\left(\mathcal{L} \otimes \omega_{\pi}\right)$, 


\section{Flavia Poma, Mattia Talpo and Fabio Tonini}

except when $g=2$, in which case there is a single relation given by $\left(\operatorname{det} \pi_{*} \omega_{\pi}\right)^{10}$. Moreover, for all $n, k \geqslant 1$, we have an isomorphism

$$
\operatorname{det} \pi_{*}\left(\mathcal{L}^{n} \otimes \omega_{\pi}^{k}\right) \simeq\left(\operatorname{det} \pi_{*} \omega_{\pi}\right)^{6 k^{2}-6 k-n^{2}+1} \otimes d_{\pi}(\mathcal{L})^{-n k+n(n+1) / 2} \otimes\left(\operatorname{det} \pi_{*}\left(\mathcal{L} \otimes \omega_{\pi}\right)\right)^{n k+n(n-1) / 2} .
$$

Proof. Taking into account Remark 2.4, everything follows from [MV14, Theorem A and 5.2, Notation 1.5, Remark 5.3].

\subsection{Genus zero case}

In this subsection we consider $g=0$, while $d$ is any integer. We will prove the following.

Proposition 2.6. Let $\pi: \mathcal{P} \longrightarrow \mathcal{J} a c_{d, 0}$ be the universal curve and let $\mathcal{L}$ be the universal invertible sheaf over $\mathcal{P}$. If $d$ is even, then Pic $\mathcal{J} a c_{d, 0}$ is freely generated by $\mathcal{L}_{0}=\pi_{*}\left(\mathcal{L} \otimes \omega_{\pi}^{d / 2}\right)$ and we have an isomorphism

$$
\operatorname{det} \pi_{*}\left(\mathcal{L}^{n} \otimes \omega_{\pi}^{k}\right) \simeq \mathcal{L}_{0}^{n \max \{n d-2 k+1,0\}} \text { for all } n, k \in \mathbb{Z} .
$$

If $d$ is odd, then Pic $\mathcal{J} a c_{d, 0}$ is freely generated by $\mathcal{L}_{0}=\operatorname{det} \pi_{*}\left(\mathcal{L} \otimes \omega_{\pi}^{(d-1) / 2}\right)$ and we have an isomorphism

$$
\operatorname{det} \pi_{*}\left(\mathcal{L}^{n} \otimes \omega_{\pi}^{k}\right) \simeq \mathcal{L}_{0}^{n \max \{n d-2 k+1,0\} / 2} \text { for all } n, k \in \mathbb{Z} .
$$

We will need the following lemma, whose proof is standard and thus omitted.

Lemma 2.7. The group $\left(\mathrm{GL}_{2}\right)^{\vee}$ is freely generated by det: $\mathrm{GL}_{2} \longrightarrow \mathbb{G}_{m}$, while $\left(\mathrm{PGL}_{2}\right)^{\vee}=0$

REMARK 2.8. Let $\pi: \mathcal{P} \longrightarrow \mathcal{S}$ be a curve of genus zero over an algebraic stack and let $\mathcal{Q}$ be an invertible sheaf on $\mathcal{P}$. If $\mathcal{Q}$ has degree zero, by Lemma 1.10 it follows that $\pi_{*} \mathcal{Q}$ is an invertible sheaf, it satisfies base change and the map $\pi^{*} \pi_{*} \mathcal{Q} \longrightarrow \mathcal{Q}$ is an isomorphism because it is so on the geometric fibers.

If $\mathcal{Q}$ has degree one, by Lemma 1.10 it follows that $\pi_{*} \mathcal{Q}$ is a rank two locally free sheaf, it satisfies base change and the map $\pi^{*} \pi_{*} \mathcal{Q} \longrightarrow \mathcal{Q}$ is surjective because it is so on the geometric fibers. In particular, we obtain an isomorphism $\mathcal{P} \longrightarrow \mathbb{P}\left(\pi_{*} \mathcal{Q}\right)$ : the pull-back of $\mathcal{O}_{\mathbb{P}\left(\pi_{*} \mathcal{Q}\right)}(1)$ is $\mathcal{Q}$ and therefore we get the Euler sequence

$$
0 \longrightarrow \omega_{\pi} \otimes \mathcal{Q} \longrightarrow \pi^{*} \pi_{*} \mathcal{Q} \longrightarrow \mathcal{Q} \longrightarrow 0 \text {. }
$$

Proof of Proposition 2.6. Since $\mathcal{M}_{0} \simeq \mathrm{BPGL}_{2}$, by Remark 1.2 and Lemma 2.7 we obtain Pic $\mathcal{M}_{0}=0$. In particular, $\operatorname{det} \pi_{*}\left(\omega_{\pi}^{k}\right)$ is trivial for all $k \in \mathbb{Z}$ in Pic $\mathcal{J} a c_{d, 0}$.

Assume that $d$ is even. Tensoring by $\omega_{\pi}^{d / 2}$ yields an isomorphism $\mathcal{J} a c_{d, 0} \longrightarrow \mathcal{J} a c_{0,0}$ over $\mathcal{M}_{0}$. By Remark 2.8 we see that the functors $\mathcal{J} a c_{0,0} \longrightarrow \mathrm{B}_{\mathcal{M}_{0}} \mathbb{G}_{m}$ mapping $(P \stackrel{q}{\longrightarrow} S, \mathcal{Q})$ to $\left(P, q_{*} \mathcal{Q}\right)$ and $\mathrm{B}_{\mathcal{M}_{0}} \mathbb{G}_{m} \longrightarrow \mathcal{J} a c_{0,0}$ mapping $(P \stackrel{q}{\longrightarrow} S, \mathcal{T})$ to $\left(P, q^{*} \mathcal{T}\right)$ are quasi-inverses of each other. Moreover, by Remark 1.2 we have Pic $\mathrm{B}_{\mathcal{M}_{0}} \mathbb{G}_{m} \simeq \operatorname{Pic} \mathcal{M}_{0} \oplus \mathbb{Z} \simeq \mathbb{Z}$, generated by the invertible sheaf given by the rule $(P, \mathcal{T}) \longmapsto \mathcal{T}$. The pull-back of this sheaf via $\mathcal{J} a c_{d, 0} \simeq \mathcal{J} a c_{0,0} \simeq \mathrm{B}_{\mathcal{M}_{0}} \mathbb{G}_{m}$ is isomorphic to $\mathcal{L}_{0}$, which therefore freely generates Pic $\mathcal{J} a c_{d, 0}$. By Remark 2.8 we have $\mathcal{L} \simeq$ $\pi^{*} \mathcal{L}_{0} \otimes \omega_{\pi}^{-d / 2}$ and, using the projection formula,

$$
\operatorname{det} \pi_{*}\left(\mathcal{L}^{n} \otimes \omega_{\pi}^{k}\right) \simeq \operatorname{det}\left(\mathcal{L}_{0}^{n} \otimes \pi_{*}\left(\omega_{\pi}^{k-n d / 2}\right)\right) \simeq \mathcal{L}_{0}^{n \operatorname{rk} \pi_{*}\left(\omega_{\pi}^{k-n d / 2}\right)} .
$$

Finally, by Lemma 1.10 we see that $\mathrm{rk} \pi_{*}\left(\omega_{\pi}^{k-n d / 2}\right)=\max \{n d-2 k+1,0\}$.

Assume now that $d$ is odd. Tensoring by $\omega_{\pi}^{(d-1) / 2}$, we get an isomorphism $\mathcal{J} a c_{d, 0} \longrightarrow \mathcal{J} a c_{1,0}$. By Remark 2.8 we see that the functors $\mathcal{J} a c_{1,0} \longrightarrow$ B GL 2 mapping $(P \stackrel{q}{\longrightarrow} S, \mathcal{Q})$ to $q_{*} \mathcal{Q}$ and 


\section{StACKS OF UNIFORM CYCLIC COVERS OF CURVES AND THEIR PICARD GROUPS}

$\mathrm{B} \mathrm{GL}_{2} \longrightarrow \mathcal{J} a c_{1,0}$ mapping $\mathcal{E}$ to $\left(\mathbb{P}(\mathcal{E}), \mathcal{O}_{\mathbb{P}(\mathcal{E})}(1)\right)$ are quasi-inverses of each other. Moreover, by Remark 1.2 we have Pic B GL $2 \simeq \mathbb{Z}$ generated by the invertible sheaf given by the rule $\mathcal{E} \longmapsto \operatorname{det} \mathcal{E}$. The pull-back of this sheaf via $\mathcal{J} a c_{d, 0} \simeq \mathcal{J} a c_{1,0} \simeq \mathrm{B} \mathrm{GL} \mathrm{L}_{2}$ is isomorphic to $\mathcal{L}_{0}$. Set $\mathcal{T}=\mathcal{L} \otimes \omega_{\pi}^{(d-1) / 2}$, so that $\mathcal{L}_{0}=\operatorname{det} \pi_{*} \mathcal{T}$. Applying the determinant to the Euler sequence in Remark 2.8 associated with $\mathcal{T}$ we get an isomorphism

$$
\omega_{\pi} \simeq \pi^{*} \mathcal{L}_{0} \otimes \mathcal{T}^{-2} .
$$

Writing $\mathcal{L}^{n} \otimes \omega_{\pi}^{k}$ in terms of $\mathcal{T}$ and $\mathcal{L}_{0}$ and applying the projection formula, we obtain $\operatorname{det} \pi_{*}\left(\mathcal{L}^{n} \otimes \omega_{\pi}^{k}\right) \simeq \operatorname{det}\left(\mathcal{L}_{0}^{k-n(d-1) / 2} \otimes \pi_{*}\left(\mathcal{T}^{n d-2 k}\right)\right) \simeq \mathcal{L}_{0}^{(k-n(d-1) / 2) \operatorname{rk} \pi_{*}\left(\mathcal{T}^{n d-2 k}\right)} \otimes \operatorname{det} \pi_{*}\left(\mathcal{T}^{n d-2 k}\right)$. By Lemma 1.10 we have $\operatorname{rk} \pi_{*}\left(\mathcal{T}^{n d-2 k}\right)=\max \{n d-2 k+1,0\}$. Thus it suffices to prove the expression $\operatorname{det} \pi_{*}\left(\mathcal{T}^{q}\right) \simeq \mathcal{L}_{0}^{q(q+1) / 2}$ for $q \geqslant 0$. Considering the Euler sequence in Remark 2.8 associated with $\mathcal{T}$, replacing $\omega_{\pi}$ by $\pi^{*} \mathcal{L}_{0} \otimes \mathcal{T}^{-2}$ and tensoring by $\mathcal{T}^{q}$, we get an exact sequence

$$
0 \longrightarrow \pi^{*} \mathcal{L}_{0} \otimes \mathcal{T}^{q-1} \longrightarrow \pi^{*} \pi_{*} \mathcal{T} \otimes \mathcal{T}^{q} \longrightarrow \mathcal{T}^{q+1} \longrightarrow 0
$$

The push-forward $\pi_{*}$ of the sequence above for $q \geqslant 0$ is exact because $\mathrm{R}^{1} \pi_{*}\left(\pi^{*} \mathcal{L}_{0} \otimes \mathcal{T}^{q-1}\right)=0$ thanks to Lemma 1.10. Thus applying $\pi_{*}$, the determinant, the projection formula and using that $\operatorname{rk} \pi_{*}\left(\mathcal{T}^{r}\right)=r+1$ for $r \geqslant-1$, we get an isomorphism

$$
\operatorname{det} \pi_{*}\left(\mathcal{T}^{q+1}\right) \simeq \mathcal{L}_{0} \otimes\left(\operatorname{det} \pi_{*}\left(\mathcal{T}^{q}\right)\right)^{2} \otimes\left(\operatorname{det} \pi_{*}\left(\mathcal{T}^{q-1}\right)\right)^{-1} .
$$

It is now easy to check by induction that $\operatorname{det} \pi_{*}\left(\mathcal{T}^{q}\right) \simeq \mathcal{L}_{0}^{q(q+1) / 2}$.

\subsection{Genus one case}

In this subsection we consider $g=1$ and $d>0$. We will prove the following theorem.

TheOREm 2.9. Let $\pi: \mathcal{E} \longrightarrow \mathcal{J} a c_{d, 1}$ be the universal curve over $\mathcal{J} a c_{d, 1}$, let $\mathcal{L}$ be the universal invertible sheaf over $\mathcal{E}$ and assume $p \nmid d$. Then Pic $\mathcal{J} a c_{d, 1}$ is generated by $\pi_{*} \omega_{\pi}$ and $\operatorname{det} \pi_{*} \mathcal{L}$ with the only relation $\left(\pi_{*} \omega_{\pi}\right)^{12}$. Moreover, we have an isomorphism

$$
\operatorname{det} \pi_{*}\left(\mathcal{L}^{n} \otimes \omega_{\pi}^{k}\right) \simeq\left(\operatorname{det} \pi_{*} \mathcal{L}\right)^{n^{2}} \otimes\left(\pi_{*} \omega_{\pi}\right)^{d n k+(n-1)(d n-2 n-2) / 2} \text { for } n>0, k \in \mathbb{Z} .
$$

The starting point is the well-known Theorem of Mumford, later generalized by Fulton and Olsson. (See [Mum63] and [FO10])

Theorem 2.10 (Mumford, Fulton, Olsson). The Picard group of $\mathcal{M}_{1,1}$ is cyclic of order 12; it is generated by $\pi_{*} \omega_{\pi}$, where $\pi: \mathcal{E} \longrightarrow \mathcal{M}_{1,1}$ is the universal curve.

We will proceed by showing that $\mathcal{J} a c_{d, 1}$ is isomorphic to $\mathrm{B}_{\mathcal{M}_{1,1}} G_{d}$, for a certain group scheme $G_{d}$ over $\mathcal{M}_{1,1}$. In particular, we will conclude that Pic $\mathcal{J} a c_{d, 1} \simeq \operatorname{Pic} \mathcal{M}_{1,1} \oplus \operatorname{Hom}\left(G_{d}, \mathbb{G}_{m}\right)$ and we will conclude the section by computing the group of characters $\operatorname{Hom}\left(G_{d}, \mathbb{G}_{m}\right)$.

Lemma 2.11. Let $\pi: \mathcal{E} \longrightarrow \mathcal{S}$ be a genus one curve over an algebraic stack. Then the functor

$$
\begin{gathered}
\mathcal{E} \longrightarrow{ }^{\Omega} \underline{\operatorname{Pic}}_{\mathcal{E} / \mathcal{S}}^{1} \\
\left(\delta: T \longrightarrow \mathcal{E} \times_{\mathcal{S}} T\right) \longmapsto\left[\mathcal{O}_{\mathcal{E} \times{ }_{\mathcal{S}} T}(\delta)\right]
\end{gathered}
$$

is an isomorphism. If $\sigma: \mathcal{S} \longrightarrow \mathcal{E}$ is a section, then also the functor

$$
\begin{gathered}
\left.\mathcal{E} \longrightarrow \times_{\mathcal{S}} T\right) \longmapsto\left[\mathcal{O}_{\mathcal{E} \times \mathcal{S}_{T} T}\left(\delta-\sigma \times_{\mathcal{S}}^{0} T\right)\right] \\
(\delta: T \longrightarrow \mathcal{E}
\end{gathered}
$$




\section{Flavia Poma, Mattia Talpo and Fabio Tonini}

is an isomorphism that sends $\sigma$ to $\left[\mathcal{O}_{\mathcal{E}}\right]$

Proof. The last part of the statement follows from the first one. We start by showing that $\Omega$ is an fppf epimorphism. Let $\chi \in \underline{\operatorname{Pic}}_{\mathcal{E}}^{1}(T)$, where $T$ is an $\mathcal{S}$-scheme. We may replace $T$ by $\mathcal{S}$ and assume the existence of an invertible sheaf $\mathcal{L}$ of degree one over $\mathcal{E}$ such that $\chi=[\mathcal{L}]$. By Lemma 1.10 the sheaf $\mathcal{Q}=\pi_{*} \mathcal{L}$ is invertible and we may assume that it is trivial. Since $\pi_{*} \mathcal{L}$ satisfies base change, the section $s$ of $\mathcal{L}$ corresponding to $1 \in \pi_{*} \mathcal{L}$ is always nonzero on the geometric fibers of $\pi$. Thus, by Lemma 1.11, we get a section $\tau: \mathcal{S} \longrightarrow \mathcal{E}$ with an isomorphism $\mathcal{O}_{\mathcal{E}}(\tau) \simeq \mathcal{L}$, so that $\Omega(\tau)=\chi$.

We now prove that $\Omega$ is injective over an $\mathcal{S}$-scheme $T$. Again, we may replace $T$ by $\mathcal{S}$, so that, in particular, $\mathcal{S}$ is a scheme. Let $\delta, \delta^{\prime} \in \mathcal{E}(\mathcal{S})$ such that $\left[\mathcal{O}_{\mathcal{E}}\left(\delta^{\prime}\right)\right]=\left[\mathcal{O}_{\mathcal{E}}(\delta)\right]$. By Lemma 1.10 we have an isomorphism

$$
\mathcal{O}_{\mathcal{E}}(\delta) \stackrel{\alpha}{\longrightarrow} \mathcal{O}_{\mathcal{E}}\left(\delta^{\prime}\right) \otimes \pi^{*} \mathcal{Q}
$$

for some invertible sheaf $\mathcal{Q}$ over $\mathcal{S}$. Since $\pi_{*} \mathcal{O}_{\mathcal{E}}(\delta)$ and $\pi_{*} \mathcal{O}_{\mathcal{E}}\left(\delta^{\prime}\right)$ are freely generated by the respective sections 1 by Lemma 1.11, applying $\pi_{*}$ we get an element $x \in \mathcal{Q}$ such that $\alpha(1)=$ $1 \otimes \pi^{*} x$ and that freely generates $\mathcal{Q}$. Thus we obtain an isomorphism $\mathcal{O}_{\mathcal{E}}(\delta) \longrightarrow \mathcal{O}_{\mathcal{E}}\left(\delta^{\prime}\right)$ mapping 1 to 1 and therefore the equality $\delta=\delta^{\prime}$.

Proposition 2.12. Let $\mathcal{E} \stackrel{\pi}{\longrightarrow} \mathcal{S}$ be a genus one curve over an algebraic stack with a section $\sigma$. Then we have isomorphisms

$$
\operatorname{det} \pi_{*} \mathcal{O}_{\mathcal{E}}(n \sigma) \simeq \sigma^{*} \mathcal{O}_{\mathcal{E}}(\sigma)^{n(n+1) / 2-1} \text { for } n \geqslant 1
$$

and

$$
\sigma^{*} \mathcal{O}_{\mathcal{E}}(\sigma) \simeq\left(\pi_{*} \omega_{\pi}\right)^{\vee}
$$

Moreover, if $\mathcal{L}$ is an invertible sheaf over $\mathcal{E}$ of degree greater than zero and $\tau$ is another section (possibly equal to $\sigma$ ), we have an isomorphism

$$
\operatorname{det} \pi_{*}\left(\mathcal{L} \otimes \mathcal{O}_{\mathcal{E}}(\tau)\right) \simeq \operatorname{det} \pi_{*}\left(\mathcal{L} \otimes \mathcal{O}_{\mathcal{E}}(\tau-\sigma)\right) \otimes \sigma^{*} \mathcal{L} \otimes \sigma^{*} \mathcal{O}_{\mathcal{E}}(\tau)
$$

Proof. In what follows we will use Lemma 1.10 and, in particular, the isomorphism $\omega_{\pi} \simeq \pi^{*} \pi_{*} \omega_{\pi}$ without further comments. Notice that the first isomorphism in the statement follows from the last one with $\tau=\sigma$, induction and the isomorphism $\pi_{*} \mathcal{O}_{\mathcal{E}}(\sigma) \simeq \mathcal{O}_{\mathcal{S}}$ (see Lemma 1.11).

Consider an invertible sheaf $\mathcal{L}$ on $\mathcal{E}$ of degree greater than or equal to zero and a section $\tau$ of $\mathcal{E}$. Tensoring by $\mathcal{L} \otimes \mathcal{O}_{\mathcal{E}}(\tau)$ the exact sequence on $\mathcal{E}$

$$
0 \longrightarrow \mathcal{O}_{\mathcal{E}}(-\sigma) \longrightarrow \mathcal{O}_{\mathcal{E}} \longrightarrow \sigma_{*} \mathcal{O}_{\mathcal{S}} \longrightarrow 0
$$

and applying $\pi_{*}$, we get an exact sequence

$0 \longrightarrow \pi_{*}\left(\mathcal{L} \otimes \mathcal{O}_{\mathcal{E}}(\tau-\sigma)\right) \longrightarrow \pi_{*}\left(\mathcal{L} \otimes \mathcal{O}_{\mathcal{E}}(\tau)\right) \longrightarrow \sigma^{*}\left(\mathcal{L} \otimes \mathcal{O}_{\mathcal{E}}(\tau)\right) \longrightarrow \mathrm{R}^{1} \pi_{*}\left(\mathcal{L} \otimes \mathcal{O}_{\mathcal{E}}(\tau-\sigma)\right) \longrightarrow 0$.

If $\mathcal{L}=\mathcal{O}_{\mathcal{E}}$ and $\tau=\sigma$, we obtain a surjection $\sigma^{*} \mathcal{O}_{\mathcal{E}}(\sigma) \longrightarrow \mathrm{R}^{1} \pi_{*} \mathcal{O}_{\mathcal{E}} \simeq\left(\pi_{*} \omega_{\pi}\right)^{\vee}$ which is therefore an isomorphism. If $\mathcal{L}$ has degree strictly greater than zero, the last term in the sequence is zero and taking the determinant we get the last isomorphism.

Proposition 2.13. The functor

$$
\begin{aligned}
& \mathcal{M}_{1,1} \longrightarrow \mathcal{J} a c_{d, 1} \\
& (E, \sigma) \longmapsto\left(E, \mathcal{O}_{E}(d \sigma)\right)
\end{aligned}
$$




\section{Stacks OF UNiform CYClic COVERS OF CURVES AND THEIR PICARD GROUPS}

is an epimorphism in the fppf topology and is a section of the functor

$$
\begin{aligned}
& \mathcal{J} a c_{d, 1} \longrightarrow \mathcal{M}_{1,1} \\
& (E, \mathcal{L}) \longmapsto\left({\underline{\mathrm{Pic}_{E / S}^{0}}}_{E},\left[\mathcal{O}_{E}\right]\right) .
\end{aligned}
$$

Proof. The second part of the statement follows from Lemma 2.11. For the first one let $(E, \mathcal{L}) \in$ $\mathcal{J} a c_{d, 1}$. By Remark 1.12 fppf locally we can write $\mathcal{L} \simeq \mathcal{T}^{d}$ for some degree one invertible sheaf $\mathcal{T}$ on $E$. By Lemma $2.11 E$ has a section $\sigma$ such that $[\mathcal{T}]=\left[\mathcal{O}_{E}(\sigma)\right]$, which means that $\mathcal{T}$ and $\mathcal{L}$ are fppf locally isomorphic to $\mathcal{O}_{E}(\sigma)$ and $\mathcal{O}_{E}(d \sigma)$, respectively.

Lemma 2.14. Let $p: \mathcal{X} \longrightarrow \mathcal{Y}$ be a map of stacks over a scheme $S$ with a section $s: \mathcal{Y} \longrightarrow \mathcal{X}$ which is an fppf epimorphism and denote by $G$ the sheaf of groups over $\mathcal{Y}$ defined by

$$
G(T \stackrel{\xi}{\longrightarrow} \mathcal{Y})=\operatorname{Ker}\left(\operatorname{Aut}_{\mathcal{X}}(s(\xi)) \longrightarrow \operatorname{Aut}_{\mathcal{Y}}(p s(\xi))\right) .
$$

Then the functor $F: \mathcal{X} \longrightarrow \mathrm{B} \mathcal{Y} G$ which maps $\eta: T \longrightarrow \mathcal{X}$ to the sheaf $F(\eta)$ over $T$ given by the inverse image of the identity section of $\underline{\operatorname{Aut}}_{T}(p(\eta))$ along the map

$$
\underline{\mathrm{IsO}}_{T}(\eta, s p(\eta)) \stackrel{p}{\longrightarrow} \underline{\operatorname{IsO}}_{T}(p(\eta), p s(p(\eta))) \simeq \underline{\operatorname{Aut}}_{T}(p(\eta))
$$

defines an equivalence of stacks.

Proof. Given $\xi: T \longrightarrow \mathcal{Y}$, denote by $\mathcal{X}_{\xi}, G_{\xi}$ and $s_{\xi}: T \longrightarrow \mathcal{X}_{\xi}$ the base change of $\mathcal{X}, G$ and $s: \mathcal{Y} \longrightarrow \mathcal{X}$ along $\xi$, respectively. Given $\eta: T \longrightarrow \mathcal{X}$, we show that $F(\eta)$ is a $G$-torsor. A direct check shows that $F(\eta)$ coincides with $\underline{\operatorname{Iso}}_{\mathcal{X}_{p(\eta)}}\left(\eta, s_{p(\eta)}\right)$ and $G_{\xi} \simeq \underline{\operatorname{Aut}}_{\mathcal{X}_{\xi}}\left(s_{\xi}\right)$. Since $\eta$ and $s_{p(\eta)}$ are fppf locally isomorphic, it follows that $F(\eta)$ is a $G_{p(\eta)}$-torsor. It is easy to see that the association $\eta \longmapsto F(\eta)$ defines a functor $F: \mathcal{X} \longrightarrow \mathrm{B}_{\mathcal{Y}} G$. Since the base change of $F$ along any morphism $T \longrightarrow \mathcal{Y}$ is an equivalence by standard results, we obtain that it is globally an equivalence.

Definition 2.15. We define the group functor $G_{d}$ over $\mathcal{M}_{1,1}$ as the group $G$ obtained as in Lemma 2.14 with respects to the maps defined in Proposition 2.13.

Let us describe the group $G_{d}$ more concretely.

Proposition 2.16. An element of $G_{d}\left(S \stackrel{(E, \sigma)}{\longrightarrow} \mathcal{M}_{1,1}\right)$ is a pair $(f, \lambda)$ where $f: E \longrightarrow E$ is a translation by an element in $E[d]$ and $\lambda: \mathcal{O}_{E}(\sigma)^{d} \longrightarrow \mathcal{O}_{E}(f(\sigma))^{d}$ is an isomorphism. Moreover, we have an exact sequence

$$
\begin{aligned}
(f, \lambda) \longmapsto f(\sigma) & \longrightarrow G_{d} \longrightarrow \mathcal{E}[d] \longrightarrow 0 \\
0 \longrightarrow \mathbb{G}_{m} \longrightarrow(\mathrm{id}, \mu) &
\end{aligned}
$$

in the Zariski topology of $\mathrm{Sch} / \mathcal{M}_{1,1}$, where $\mathcal{E} \longrightarrow \mathcal{M}_{1,1}$ is the universal curve. In particular, $G_{d}$ is affine and of finite type over $\mathcal{M}_{1,1}$ and, if $p \nmid d$, it is smooth over $\mathcal{M}_{1,1}$.

Proof. By definition, an element of $G_{d}\left(S \stackrel{(E, \sigma)}{\longrightarrow} \mathcal{M}_{1,1}\right)$ is a pair $(f, \lambda)$ where $f: E \longrightarrow E$ is an isomorphism such that $f_{*}: \underline{\mathrm{Pic}}_{E / S}^{0} \longrightarrow \underline{\mathrm{Pic}}_{E / S}^{0}$ is the identity and $\lambda: \mathcal{O}_{E}(\sigma)^{d} \longrightarrow \mathcal{O}_{E}(f(\sigma))^{d}$ is an isomorphism. Taking into account Lemma 2.11, the condition $f_{*}=$ id means that, for all $\delta \in \mathcal{E}(T)$ and $S$-scheme $T$, we have

$$
\left[\mathcal{O}_{E_{T}}(f(\delta)-f(\sigma))\right]=\left[\mathcal{O}_{E_{T}}(\delta-\sigma)\right],
$$




\section{Flavia Poma, Mattia Talpo and Fabio Tonini}

which implies that $f: E \longrightarrow E$ is a translation. The existence of $\lambda$ also implies that $f(\sigma) \in E[d]$. In particular, the sequence in the statement is well defined and, since $\pi_{*} \mathbb{G}_{m, E} \simeq \mathbb{G}_{m, S}$, it is exact in the first two terms.

It remains to prove that $G_{d} \longrightarrow \mathcal{E}[d]$ is a Zariski epimorphism. This will also imply that $G_{d}$ is locally a product of $\mathbb{G}_{m}$ and $\mathcal{E}[d]$, proving the last sentence in the statement. Let $\delta \in \mathcal{E}[d]$ and let $t$ be the translation by $\delta$, so that $t(\sigma)=\delta$. By Lemma 1.10, the equality $\left[\mathcal{O}_{E}(\delta-\sigma)^{d}\right]=0$ in $\underline{\operatorname{Pic}}_{E / S}^{0}$ implies that $\mathcal{O}_{E}(\delta-\sigma)^{d} \simeq \pi^{*} \mathcal{Q}$, where $\mathcal{Q}$ is an invertible sheaf over $S$. In particular, where $\mathcal{Q}$ is trivial, we get an isomorphism $\lambda: \mathcal{O}_{E}(\sigma-t(\sigma))^{d} \longrightarrow \mathcal{O}_{E}$ and therefore a pair $(t, \lambda) \in G_{d}$ over $\delta$.

The groups obtained from $G_{d}$ as base change along geometric points $\operatorname{Spec} k \stackrel{(E, p)}{\longrightarrow} \mathcal{M}_{1,1}$ are particular cases of Theta groups, first defined by Mumford in his paper [Mum66]. With notation from this paper we have $G_{d} \times \mathcal{M}_{1,1} k=\mathcal{G}\left(\mathcal{O}_{E}(d p)\right)$.

COROLlary 2.17. We have an isomorphism $\mathcal{J}_{a c_{d, 1}} \simeq \mathrm{B}_{\mathcal{M}_{1,1}} G_{d}$.

By Remark 1.2 the last step in the computation of the Picard group of $\mathcal{J} a c_{d, 1}$ is the study of the group of characters $\operatorname{Hom}\left(G_{d}, \mathbb{G}_{m}\right)$. For the remaining part of this subsection we assume $p \nmid d$.

LEMMA 2.18. The map

$$
\begin{aligned}
G_{d} \times G_{d} \stackrel{\hat{e}_{d}}{\longrightarrow} \mathbb{G}_{m} \\
(x, y) \longmapsto x y x^{-1} y^{-1}
\end{aligned}
$$

is bilinear and factors through a map $e_{d}: \mathcal{E}[d] \times \mathcal{E}[d] \longrightarrow \mathbb{G}_{m}$, where $\mathcal{E} \longrightarrow \mathcal{M}_{1,1}$ is the universal curve. Moreover, the map $e_{d}$ induces an isomorphism

$$
\mathcal{E}[d] \simeq \underline{\operatorname{Hom}}\left(\mathcal{E}[d], \mathbb{G}_{m}\right) .
$$

Proof. The map $\hat{e}_{d}$ is well defined because $\mathcal{E}[d]$ is abelian. Note that $\mathbb{G}_{m} \subseteq G_{d}$ is contained in the center. In particular, the map $e_{d}$ in the statement is well defined and we have

$$
\hat{e}_{d}(x y, z)=x y z y^{-1} x^{-1} z^{-1}=x \hat{e}_{d}(y, z) z x^{-1} z^{-1}=\hat{e}_{d}(x, z) \hat{e}_{d}(y, z) .
$$

Finally, $\hat{e}_{d}(x, y)=\hat{e}_{d}(y, x)^{-1}$ and $\hat{e}_{d}$ and $e_{d}$ are therefore bilinear. Let $\phi$ be the induced map $\mathcal{E}[d] \longrightarrow \underline{\operatorname{Hom}}\left(\mathcal{E}[d], \mathbb{G}_{m}\right)$. This is a map between flat and finite group schemes and we can check that it is an isomorphism on the geometric points. So let $E$ be an elliptic curve over an algebraically closed field. In this case $E[d] \simeq \mathbb{Z} / d \mathbb{Z} \times \mathbb{Z} / d \mathbb{Z}$ and $\underline{\operatorname{Hom}}\left(E[d], \mathbb{G}_{m}\right) \simeq E[d]$ as abstract groups. The result then follows from the fact that $e_{d}$ is nondegenerate thanks to [Mum66, §1, Theorem 1].

Proposition 2.19. Let $\mathcal{E} \longrightarrow \mathcal{M}_{1,1}$ be the universal curve. Then

$$
\mathcal{E}[d]^{\vee}=\operatorname{Hom}\left(\mathcal{E}[d], \mathbb{G}_{m}\right)=0 .
$$

In particular, the map $G_{d}^{\vee} \longrightarrow \mathbb{G}_{m}^{\vee}$ induced by the inclusion $\mathbb{G}_{m} \longrightarrow G_{d}$ is injective.

Proof. By Lemma 2.18 we have $\underline{\operatorname{Hom}}\left(\mathcal{E}[d], \mathbb{G}_{m}\right) \simeq \mathcal{E}[d]$. Therefore we have to prove that there are no sections $\mathcal{M}_{1,1} \longrightarrow \mathcal{E}[d]$ but the zero one. By contradiction, assume we have a nonzero section $\delta: \mathcal{M}_{1,1} \longrightarrow \mathcal{E}[d]$. In particular, by base change, we have a section $\delta_{E}$ for all elliptic curves $E$. Since $\mathcal{E}[d]$ is étale and separated over $\mathcal{M}_{1,1}$, there are no elliptic curves $E$ such that $\delta_{E}$ is zero. Moreover, we may assume that $d$ is prime. If $d=2$, let $k$ be a field having an irreducible and separable polynomial $g \in k[x]$ of degree 3 and consider the elliptic curve defined by the equation 


\section{StACKS OF UNIFORM CYCLIC COVERS OF CURVES AND THEIR PICARD GROUPS}

$y^{2}=g(x)$. In this case $E[2](k)=0$ by [Sil86, Group law algorithm 2.3] because $g$ has no zeros in $k$ and therefore $\delta_{E}=0$, which is a contradiction.

So we may assume that $d$ is an odd prime. Let $k$ be a field having a degree two separable extension $L$ and an elliptic curve $E$. We are going to prove that $\delta_{E} \in E(k)$ is invariant under the involution of $E$, that is, that $\delta_{E} \in E[2](k)$. This will end the proof because $E[d](k) \cap E[2](k)=0$.

We want to construct a new elliptic curve $E^{\prime}$ over $k$ with the following construction. Let $F$ be a sheaf of sets over a scheme $S$ with an involution $i: F \longrightarrow F$, let $P \longrightarrow S$ be a $\mathbb{Z} / 2 \mathbb{Z}$-torsor and call $\sigma: P \longrightarrow P$ the induced order two automorphism. We define a new functor by

$$
F_{P / S, i}:(\mathrm{Sch} / S)^{\mathrm{op}} \longrightarrow(\mathrm{sets}), F_{P / S, i}(T)=\left\{x \in F(T \times P) \mid \sigma^{*} x=i(x)\right\} .
$$

Since $i$ and $\sigma^{*}$ commute, it is easy to check that $F_{P / S, i}$ is a sheaf and that if $S^{\prime} \longrightarrow S$ is any base change, then $F_{P / S, i} \times S^{\prime}=F_{P \times S^{\prime} / S^{\prime}, i}$. Moreover, if $\Delta: T \longrightarrow P \times T$ is a section, it is easy to check that

$$
F_{P / S, i}(T) \longrightarrow F(T), z \longmapsto \Delta^{*} z
$$

is a bijection. In particular, for any torsor $P$ we get an isomorphism $\tau: F_{P / S, i} \times P \longrightarrow F \times P$ induced by the diagonal section $P \longrightarrow P \times P$. We now claim that if there exist $x \in F_{P / S, i}(S)$ and $y \in F(S)$ such that $\tau\left(h^{*} x\right)=h^{*} y$, where $h: P \longrightarrow S$ is the structure morphism, then it follows that $i(y)=y$. Indeed, by construction $\tau\left(h^{*} x\right)=x, \sigma^{*} h^{*} y=h^{*} y$ and, since $x \in F_{P / S, i}(S)$, $\sigma^{*} x=i(x)$. In particular, $h^{*} i(y)=h^{*} y$ and by descent $i(y)=y$.

We apply the previous construction with $F=E, i$ the involution of $E$ and $P=\operatorname{Spec} L$. Set $E^{\prime}=F_{P / k, i}$. The sheaf $E^{\prime}$ is a genus one curve and $0 \in E^{\prime}(k) \subseteq E(L)$. Thus $E^{\prime}$ is an elliptic curve and, by construction, $\tau: E^{\prime} \times P \longrightarrow E \times P$ preserves the neutral element; that is, it is an isomorphism of elliptic curves. Since $\delta_{E}$ and $\delta_{E^{\prime}}$ come from a global section on $\mathcal{M}_{1,1}$ we should have $\tau\left(\delta_{E^{\prime} \times P}\right)=\delta_{E \times P}$, which implies that $i\left(\delta_{E}\right)=\delta_{E}$ as shown above.

Proposition 2.20. Consider the map $G_{d}^{\vee} \longrightarrow \mathbb{G}_{m}^{\vee}=\mathbb{Z}$ induced by the inclusion $\mathbb{G}_{m} \longrightarrow G_{d}$. The morphism

$$
\operatorname{Pic} \mathcal{J} a c_{d, 1} \simeq \operatorname{Pic} \mathcal{M}_{1,1} \oplus G_{d}^{\vee} \longrightarrow \operatorname{Pic} \mathcal{M}_{1,1} \oplus \mathbb{Z} \simeq \mathbb{Z} / 12 \mathbb{Z} \oplus \mathbb{Z}
$$

sends the invertible sheaf on $\mathcal{J} a c_{d, 1}$ defined by $\mathcal{J} a c_{d, 1} \ni(E \stackrel{\pi}{\longrightarrow} S, \mathcal{L}) \longmapsto \operatorname{det} \pi_{*} \mathcal{L}^{n}$ to the element $\left(1-n d(n d+1) / 2, n^{2} d\right)$

Proof. Notice that $\mathrm{B}_{\mathcal{M}_{1,1}} \mathbb{G}_{m}$ is isomorphic to the stack $\mathcal{X}$ of triples $(E \stackrel{\pi}{\longrightarrow} S, \sigma, \mathcal{Q})$ where $(E, \sigma) \in \mathcal{M}_{1,1}$ and $\mathcal{Q}$ is an invertible sheaf over $S$. Consider the functor

$$
\begin{gathered}
\mathcal{X} \stackrel{\Omega}{\longrightarrow} \mathcal{J} a c_{d, 1} \\
(E \stackrel{\pi}{\longrightarrow} S, \sigma, \mathcal{Q}) \longmapsto\left(E \stackrel{\pi}{\longrightarrow} S, \mathcal{O}_{E}(d \sigma) \otimes \pi^{*} \mathcal{Q}\right) .
\end{gathered}
$$

We claim that the functor $\widetilde{\Omega}: \mathrm{B}_{\mathcal{M}_{1,1}} \mathbb{G}_{m} \simeq \mathcal{X} \longrightarrow \mathcal{J} a c_{d, 1} \simeq \mathrm{B}_{\mathcal{M}_{1,1}} G_{d}$ is naturally equivalent to the map $\beta_{*}: \mathrm{B}_{\mathcal{M}_{1,1}} \mathbb{G}_{m} \longrightarrow \mathrm{B}_{\mathcal{M}_{1,1}} G_{d}$ induced by the inclusion $\mathbb{G}_{m} \longrightarrow G_{d}$. Given $(E \stackrel{\pi}{\longrightarrow} S, \sigma, \mathcal{Q}) \in \mathcal{X}$, the associated $\mathbb{G}_{m}$-torsor is $\underline{\operatorname{Iso}}_{S}\left(\mathcal{Q}, \mathcal{O}_{S}\right)$, while $\widetilde{\Omega}\left(\underline{\operatorname{IsO}}_{S}\left(\mathcal{Q}, \mathcal{O}_{S}\right)\right)$ is the subsheaf of

$$
\underline{\text { Iso }}_{\mathcal{J} a c_{d, 1}}\left(\left(E, \mathcal{O}_{E}(d \sigma) \otimes \pi^{*} \mathcal{Q}\right),\left(E, \mathcal{O}_{E}(d \sigma)\right)\right)
$$

of isomorphism $(f, \lambda)$ such that $f_{*}: \operatorname{Pic}_{E}^{0} \longrightarrow \operatorname{Pic}_{E}^{0}$ is the identity by Lemma 2.14. In particular, there is a $\mathbb{G}_{m}$-equivariant map $\underline{\mathrm{Iso}}_{S}\left(\mathcal{Q}, \mathcal{O}_{S}\right) \longrightarrow \widetilde{\Omega}\left(\underline{\operatorname{IsO}}_{S}\left(\mathcal{Q}, \mathcal{O}_{S}\right)\right)$ compatible with base changes and automorphisms of $\mathcal{Q}$. Recall that $\beta_{*}(P)=\left(P \times G_{d}\right) / \mathbb{G}_{m}$ for a $\mathbb{G}_{m}$-torsor $P$ and that, if $Q$ is a 


\section{Flavia Poma, Mattia Talpo and Fabio Tonini}

$G_{d^{-}}$torsor, $\mathbb{G}_{m}$-equivariant maps $P \longrightarrow Q$ are in one to one correspondence with $G_{d^{-}}$-equivariant isomorphism $\beta_{*}(P) \longrightarrow Q$. Thus given $P$, the $\mathbb{G}_{m}$-equivariant map $P \longrightarrow \widetilde{\Omega}(P)$ induces an isomorphism $\beta_{*}(P) \longrightarrow \widetilde{\Omega}(P)$ of $G_{d^{-}}$torsors and it is easy to check that this yields a natural isomorphism $\beta_{*} \longrightarrow \widetilde{\Omega}$.

We can conclude that the map in the statement can be seen as the pull-back along $\Omega: \mathcal{X} \longrightarrow$ $\mathcal{J} a c_{d, 1}$ on the Picard groups. The invertible sheaf defined in the statement is sent to

$$
(E \stackrel{\pi}{\longrightarrow} S, \sigma, \mathcal{Q}) \longmapsto \operatorname{det} \pi_{*}\left(\mathcal{O}_{E}(n d \sigma) \otimes \pi^{*} \mathcal{Q}^{n}\right) \simeq\left(\operatorname{det} \pi_{*} \mathcal{O}_{E}(n d \sigma)\right) \otimes \mathcal{Q}^{n^{2} d}
$$

Since the invertible sheaf $(E, \sigma, \mathcal{Q}) \longmapsto \mathcal{Q}$ is the generator of $\mathbb{G}_{m}^{\vee}$ in Pic $\mathrm{B}_{\mathcal{M}_{1,1}} \mathbb{G}_{m}$, the result follows from Proposition 2.12.

Proposition 2.21. The image of the map $G_{d}^{\vee} \longrightarrow \mathbb{G}_{m}^{\vee} \simeq \mathbb{Z}$ induced by the inclusion $\mathbb{G}_{m} \longrightarrow G_{d}$ is $d \mathbb{Z}$.

Proof. Call $\alpha: G_{d}^{\vee} \longrightarrow \mathbb{G}_{m}^{\vee} \simeq \mathbb{Z}$ the map in the statement. By Proposition 2.20 applied to $n=1$, we see that $d \mathbb{Z} \subseteq \operatorname{Im} \alpha$. Let $E$ be an elliptic curve over an algebraically closed field $k$. By Lemma 2.18, it follows that there exist $x, y \in G_{d}(k)$ such that $\omega=e_{d}(x, y)=x y x^{-1} y^{-1}$ is a primitive $d$ th root of unity. If $\psi: G_{d} \longrightarrow \mathbb{G}_{m}$ is a morphism and we set $r=\alpha(\psi)$, we have $1=\psi(\omega)=\omega^{r}$, which implies $d \mid r$.

Proof of Theorem 2.9. By Corollary 2.17 we have $\mathcal{J} a c_{d, 1} \simeq \mathrm{B}_{\mathcal{M}_{1,1}} G_{d}$ and by Remark 1.2 we can conclude that Pic $\mathcal{J} a c_{d, 1} \simeq \operatorname{Pic} \mathcal{M}_{1,1} \oplus G_{d}^{\vee}$. By Propositions 2.19 and 2.21 we have $G_{d}^{\vee}=d \mathbb{G}_{m}^{\vee} \subseteq$ $\mathbb{G}_{m}^{\vee}$ and by Proposition 2.20 that

$$
\mathcal{T}=\operatorname{det} \pi_{*} \mathcal{L} \otimes\left(\pi_{*} \omega_{\pi}\right)^{d(d+1) / 2-1}
$$

freely generates $G_{d}^{\vee}$. Again by Proposition 2.20 we have

$$
\operatorname{det} \pi_{*}\left(\mathcal{L}^{n}\right) \simeq \mathcal{T}^{n^{2}} \otimes\left(\pi_{*} \omega_{\pi}\right)^{1-n d(n d+1) / 2} .
$$

Taking into account that $\omega_{\pi} \simeq \pi^{*} \pi_{*} \omega_{\pi}$ and using the projection formula, a direct computation concludes the proof.

\section{Canonical covers and their discriminant loci}

In this section we work over a field of characteristic $p \geqslant 0$ and we fix nonnegative integers $g$ and $d$ and a positive integer $n$.

Definition 3.1 . We denote by $\mathcal{V}_{d, g, n}$ (respectively $\left.\mathcal{U}_{d, g, n}\right)$ the stack of triples $(p: C \longrightarrow S, \mathcal{Q}, s)$ where $(p: C \longrightarrow S, \mathcal{Q}) \in \mathcal{J} a c_{d, g}$ and $s$ is a section of $\mathcal{Q}^{n}$ (respectively a section of $\mathcal{Q}^{n}$ that is not identically zero on any of the geometric fibers of $p: C \longrightarrow S)$.

Let $\mathcal{C} \longrightarrow \mathcal{U}_{d, g, n}$ be the universal curve of $\mathcal{U}_{d, g, n}$ and let $\mathcal{L}$ be its universal invertible sheaf. By definition, $\mathcal{L}^{n}$ carries a section; we will call it the marked section of $\mathcal{L}^{n}$.

If $d>0$, we denote by $\mathcal{H}_{d, g, n}$ the zero locus of the marked section of $\mathcal{L}^{n}$. By Lemma 1.11 the map $\mathcal{H}_{d, g, n} \longrightarrow \mathcal{U}_{d, g, n}$ is a degree $n d$ cover; we will call it the canonical cover of $\mathcal{U}_{d, g, n}$. Moreover, the closed immersion $\mathcal{H}_{d, g, n} \longrightarrow \mathcal{C}$ defines a functor $\rho_{d, g, n}: \mathcal{U}_{d, g, n} \longrightarrow$ Hilb $_{\mathcal{M}_{g, 1} / \mathcal{M}_{g}}:$ an object $(C, \mathcal{Q}, s)$ is sent to the zero locus $Z(s) \subseteq C$ of $s \in \mathcal{Q}^{n}$. Finally, we denote by $\mathcal{Z}_{d, g, n}$ the discriminant locus of the canonical cover $\mathcal{H}_{d, g, n} \longrightarrow \mathcal{U}_{d, g, n}$ of $\mathcal{U}_{d, g, n}$.

The aim of this section is to understand the geometry of the stacks $\mathcal{U}_{d, g, n}$ and $\mathcal{Z}_{d, g, n}$. We will prove the following. 


\section{StACKS OF UNIFORM CYCLIC COVERS OF CURVES AND THEIR PICARD GROUPS}

Theorem 3.2. Assume $d>0$. Let $\mathcal{C} \stackrel{\pi}{\longrightarrow} \mathcal{U}_{d, g, n}$ be the universal curve of $\mathcal{U}_{d, g, n}$ and let $\mathcal{L}$ be the universal invertible sheaf over $\mathcal{C}$. Then $\mathcal{Z}_{d, g, n} \neq \emptyset$ if and only if $d n \neq 1$ and in this case $\mathcal{Z}_{d, g, n}$ is flat and surjective over $\mathcal{M}_{g}$ and we have:

(i) The stack $\mathcal{Z}_{d, g, n}$ is the zero locus in $\mathcal{U}_{d, g, n}$ of a section of the invertible sheaf

$$
\left(\operatorname{det} \pi_{*}\left(\mathcal{L}^{n} \otimes \omega_{\pi}\right)\right)^{2} \otimes\left(\operatorname{det} \pi_{*} \omega_{\pi}\right)^{-2} .
$$

(ii) If $p \nmid n$ or $g=0$ then $\mathcal{Z}_{d, g, n}$ is geometrically reduced over $\mathcal{M}_{g}$ and, in particular, reduced.

(iii) The stack $\mathcal{Z}_{d, g, n}$ is irreducible in the following cases: $n=1$; $d n>2 g$; $d n=2 g$ and $g \geqslant 3$; $d n=2 g-1$ and $g \geqslant 4$.

(iv) If $g=1$ and $p \neq 2$, then $\mathcal{Z}_{1,1,2}$ is a disjoint union of two integral substacks of $\mathcal{U}_{1,1,2}$, one of which is the zero locus of a section of the invertible sheaf

$$
\operatorname{det}\left(\pi_{*} \mathcal{L}^{2}\right) \otimes\left(\pi_{*} \mathcal{L}\right)^{-2} .
$$

We start giving a more precise description of the stacks $\mathcal{U}_{d, g, n}$, proving, in particular, that they are algebraic and explaining the relation with the Hilbert scheme Hilb $\mathcal{M}_{g, 1} \mathcal{M}_{g}$.

REMARK 3.3. Assume $n d>2 g-2$. In this case, by Lemma 1.10, the stack $\mathcal{V}_{d, g, n}$ is a vector bundle of rank $n d+1-g$ over $\mathcal{J} a c_{d, g}$ corresponding to the locally free sheaf $\pi_{*}\left(\mathcal{L}^{n}\right)$, where $\pi: \mathcal{C} \longrightarrow \mathcal{J} a c_{d, g}$ is the universal curve and $\mathcal{L}$ is the universal invertible sheaf over $\mathcal{C}$. Moreover, $\mathcal{U}_{d, g, n}$ is the complement of the zero section of $\mathcal{V}_{d, g, n}$.

Proposition 3.4. If $d>0$, the functor $\rho_{d, g, 1}: \mathcal{U}_{d, g, 1} \longrightarrow \operatorname{Hilb}_{\mathcal{M}_{g, 1} / \mathcal{M}_{g}}$ is an equivalence. $A$ quasi-inverse is given by

$$
\begin{aligned}
\operatorname{Hilb}_{\mathcal{M}_{g, 1} / \mathcal{M}_{g}}^{d} & \longrightarrow \mathcal{U}_{d, g, 1} \\
(C, Z \subseteq C) \longmapsto & \longrightarrow\left(C, \mathcal{I}_{Z}^{\vee}, 1\right)
\end{aligned}
$$

where $\mathcal{I}_{Z}$ is the sheaf of ideals defining $Z$.

Proof. Given an object $Z \subseteq C \in \operatorname{Hilb}_{\mathcal{M}_{g, 1} \mathcal{M}_{g}}^{d}(S)$, we have to prove that $\mathcal{I}_{Z}$ is invertible over $C$. Since $\operatorname{Hilb}_{\mathcal{M}_{g, 1} / \mathcal{M}_{g}}^{d}$ is smooth over $\mathcal{M}_{g}$, we may assume that $S$ and therefore $C$ are smooth over the base field. By the flatness of $Z, I_{Z}$ is invertible on the fibers of $C \longrightarrow S$ and therefore $\operatorname{dim}_{k(p)} \mathcal{I}_{Z} \otimes k(p)=1$ for all $p \in C$, which implies that $I_{Z}$ is invertible over $C$. The discussion above shows that the functor in the statement is well defined. The fact that the functors are quasi-inverses of each other is standard.

Proposition 3.5. If $(C, \mathcal{Q}, s) \in \mathcal{U}_{0, g, n}$, then $s: \mathcal{O}_{C} \longrightarrow \mathcal{Q}^{n}$ is an isomorphism. Moreover, the functor $\mathcal{U}_{0, g, n} \longrightarrow\left(\underline{\mathrm{Pic}}_{\mathcal{M}_{g, 1} / \mathcal{M}_{g}}^{0}\right)[n]$ is a $\mu_{n}$-gerbe.

Proof. The first claim follows from the fact that a nonzero section of a degree zero invertible sheaf on a proper curve over an algebraically closed field always generates it. Denote by $F$ the functor in the statement. Given $\xi: T \longrightarrow\left(\underline{\mathrm{Pic}}_{\mathcal{M}_{g, 1} / \mathcal{M}_{g}}\right)[n]$, denote by $\mathcal{Y} \longrightarrow T$ the base change of $F$ along $\xi$. In order to prove that $\mathcal{Y}$ is a $\mu_{n}$-gerbe, we may assume that $\xi$ is given by a curve $p: C \longrightarrow T$ and the class of some invertible sheaf $\mathcal{L}$ on $C$. Since $n[\mathcal{L}]=0$ in $\underline{\text { Pic }}_{C}^{0}$, the sheaf $p_{*}\left(\mathcal{L}^{n}\right)$ is invertible by Lemma 1.10 and we may assume that it is trivial. An object of $\mathcal{Y}(T)$ is a pair $(\mathcal{Q}, s)$ where $\mathcal{Q}$ is an invertible sheaf over $C, s: \mathcal{O}_{C} \longrightarrow \mathcal{Q}^{n}$ is an isomorphism and $[\mathcal{Q}]=[\mathcal{L}]$. Those data define an invertible sheaf $\mathcal{T}=p_{*}\left(\mathcal{L} \otimes \mathcal{Q}^{-1}\right)$ with an isomorphism $\mathcal{O}_{T} \longrightarrow \mathcal{T}^{n}$. Since $T$ is arbitrary, we get a functor $\mathcal{Y} \longrightarrow \mathrm{B}_{T} \mu_{n}$ which is easily seen to be an equivalence. 


\section{Flavia Poma, Mattia Talpo and Fabio Tonini}

Proposition 3.6. The map

$$
\begin{aligned}
\mathcal{J} a c_{d, g} \stackrel{n}{\longrightarrow} \mathcal{J} a c_{d n, g} \\
(C, \mathcal{Q}) \longmapsto\left(C, \mathcal{Q}^{n}\right)
\end{aligned}
$$

is the composition of a $\mu_{n}$-gerbe followed by a surjective cover. If $p \nmid n$ or $g=0$, it is smooth.

Proof. Consider the diagram

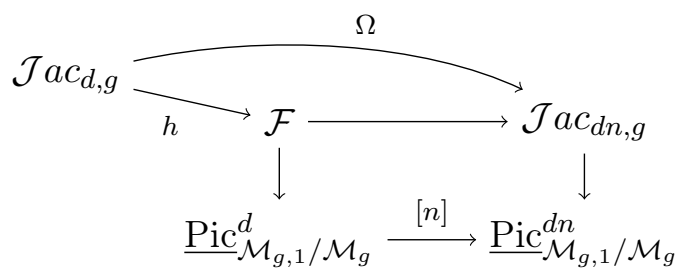

where the square diagram is Cartesian. By Remark 1.12 the map $[n]$ and therefore the map $\mathcal{F} \longrightarrow \mathcal{J} a c_{d n, g}$ are covers of degree $n^{2 g}$; they are étale if $p \nmid n$ or $g=0$. It remains to prove that $h: \mathcal{J} a c_{d, g} \longrightarrow \mathcal{F}$ is a $\mu_{n}$-gerbe. An object of $\mathcal{F}$ over a scheme $S$ is a triple $(C, \chi, \mathcal{Q})$ where $(C, \mathcal{Q}) \in \mathcal{J} a c_{d n, g}(S), \chi \in \underline{\operatorname{Pic}}_{C}^{d}(S)$ satisfying $[n] \chi=[\mathcal{Q}]$. The map $h$ sends $(C, \mathcal{T})$ to $\left(C,[\mathcal{T}], \mathcal{T}^{n}\right)$. Let $\mathcal{Y} \longrightarrow T$ be the base change of $h$ along a map $T \longrightarrow \mathcal{F}$ given by the data $(q: C \longrightarrow T, \chi, \mathcal{Q})$. The objects in $\mathcal{Y}(T)$ are pairs $(\mathcal{T}, \lambda)$ where $\mathcal{T}$ is a degree $d$ invertible sheaf on $C$ with $\chi=[\mathcal{T}]$ and $\lambda: \mathcal{T}^{n} \longrightarrow \mathcal{Q}$ is an isomorphism. We may assume $\chi=\left[\mathcal{T}_{0}\right]$ and, since $[n] \chi=[\mathcal{Q}]$ means $\left[\mathcal{T}_{0}^{n}\right]=[\mathcal{Q}]$ so that $\mathcal{T}_{0}^{n} \simeq \mathcal{Q} \otimes q^{*} \mathcal{R}$ for some invertible sheaf $\mathcal{R}$ on $T$ by Lemma 1.10, we may also assume $\mathcal{Y}(T) \neq \emptyset$. In this case $\mathcal{Y}(T)$ is isomorphic to the category of pairs $(\overline{\mathcal{T}}, \mu)$ where $\overline{\mathcal{T}}$ is a degree zero invertible sheaf with $[\overline{\mathcal{T}}]=0$ in $\underline{\mathrm{Pic}}_{C}^{0}$ and $\mu: \overline{\mathcal{T}}^{n} \longrightarrow \mathcal{O}_{C}$ is an isomorphism. It follows that $\mathcal{Y} \longrightarrow T$ is the base change of the map $\mathcal{U}_{0, g, n} \longrightarrow\left(\underline{\mathrm{Pic}}_{\mathcal{M}_{g, 1} / \mathcal{M}_{g}}\right)[n]$ along the map $T \longrightarrow\left(\underline{\operatorname{Pic}}_{\mathcal{M}_{g, 1} / \mathcal{M}_{g}}\right)[n]$ given by $\left(C,\left[\mathcal{O}_{C}\right]\right)$; it is therefore a $\mu_{n}$-gerbe (see Proposition 3.5 ).

Proposition 3.7. The stack $\mathcal{U}_{d, g, n}$ is algebraic, flat and of finite type over $\mathcal{M}_{g}$. Moreover, the map

$$
\begin{gathered}
\mathcal{U}_{d, g, n} \stackrel{\Omega}{\longrightarrow} \mathcal{U}_{d n, g, 1} \\
(C, \mathcal{Q}, s) \longmapsto\left(C, \mathcal{Q}^{n}, s\right)
\end{gathered}
$$

is flat, surjective and of finite type. If $d>0$, then $\rho_{d n, g, 1} \circ \Omega=\rho_{d, g, n}$ (see Definition 3.1 for the notation) and, in particular, $\Omega^{-1}\left(\mathcal{Z}_{d n, g, 1}\right)=\mathcal{Z}_{d, g, n}$. If $p \nmid n$ or $g=0$, then $\Omega$ is smooth and $\mathcal{U}_{d, g, n}$ is smooth over $\mathcal{M}_{g}$.

Proof. The equality $\rho_{d n, g, 1} \circ \Omega=\rho_{d, g, n}$ follows from the fact that the base change of $\mathcal{H}_{d n, g, 1} \longrightarrow$ $\mathcal{U}_{d n, g, 1}$ along $\Omega$ is $\mathcal{H}_{d, g, n} \longrightarrow \mathcal{U}_{d, g, n}$. Since the map $\Omega$ is the base change of $\mathcal{J} a c_{d, g} \longrightarrow \mathcal{J}_{a c_{d n, g}}$ (see Proposition 3.6) along the map $\mathcal{U}_{d n, g, 1} \longrightarrow \mathcal{J} a c_{d n, g}$, by Proposition 3.6 we can reduce the problem to the case $n=1$, where all the claims follow from Propositions 3.4 and 3.5.

The remaining part of the section is dedicated to the proof of Theorem 3.2. In particular in what follows we assume $d>0$.

Proof of Theorem 3.2(i). Set $\mathcal{H}_{d, g, n}=\operatorname{Spec} \mathscr{A}$. By definition $\mathcal{Z}_{d, g, n}$ is the zero locus of a section of $(\operatorname{det} \mathscr{A})^{-2}$. We have an exact sequence on $\mathcal{C}$

$$
0 \longrightarrow \mathcal{L}^{-n} \longrightarrow \mathcal{O}_{\mathcal{C}} \longrightarrow \mathcal{O}_{\mathcal{H}_{d, g, n}} \longrightarrow 0
$$




\section{StACKS OF UNIFORM CYCLIC COVERS OF CURVES AND THEIR PICARD GROUPS}

Applying $\pi_{*}$ we get an exact sequence on $\mathcal{U}_{d, g, n}$

$$
\pi_{*} \mathcal{L}^{-n}=0 \longrightarrow \mathcal{O}_{\mathcal{U}_{d, g, n}} \longrightarrow \mathscr{A} \longrightarrow \mathrm{R}^{1} \pi_{*}\left(\mathcal{L}^{-n}\right) \longrightarrow \mathrm{R}^{1} \pi_{*} \mathcal{O}_{\mathcal{C}} \longrightarrow 0=\mathrm{R}^{1} \pi_{*} \mathcal{O}_{\mathcal{H}_{d, g, n}}
$$

where the first and last vanishing follow from Lemma 1.10. We also have

$$
\mathrm{R}^{1} \pi_{*}\left(\mathcal{L}^{-n}\right) \simeq \pi_{*}\left(\mathcal{L}^{n} \otimes \omega_{\pi}\right)^{\vee} \quad \text { and } \quad \mathrm{R}^{1} \pi_{*} \mathcal{O}_{\mathcal{C}} \simeq\left(\pi_{*} \omega_{\pi}\right)^{\vee},
$$

again by Lemma 1.10. We can now deduce the following formula for the determinant of $\mathscr{A}$ :

$$
\operatorname{det} \mathscr{A} \simeq\left(\operatorname{det} \pi_{*}\left(\mathcal{L}^{n} \otimes \omega_{\pi}\right)\right)^{-1} \otimes \operatorname{det} \pi_{*} \omega_{\pi},
$$

so that det $\mathscr{A}^{-2}$ is isomorphic to the invertible sheaf in the statement.

REMARK 3.8. If $R$ is a local ring, $R \longrightarrow S$ is a flat map of rings and $M$ is an $R$-module, then

$$
\mathrm{l}_{S}\left(M \otimes_{R} S\right)=\mathrm{l}_{S}\left(S / m_{R} S\right) \mathrm{l}_{R}(M)
$$

where 1 denotes the length function.

The following theorem is one of the crucial points of this paper.

Theorem 3.9. Assume $n \geqslant 2$. The discriminant locus of the universal degree $n$ cover of Hilb $_{\mathcal{M}_{g, 1} / \mathcal{M}_{g}}$ is flat, surjective and geometrically integral over $\mathcal{M}_{g}$. In particular, it is integral.

Proof. The problem is local on $\mathcal{M}_{g}$, therefore we may replace $\mathcal{M}_{g}$ by a noetherian scheme $Y$ and $\mathcal{M}_{g, 1}$ by a genus $g$ curve $C$ over $Y$. Set $Z$ for the discriminant locus. By definition $Z$ is the zero locus of a section of an invertible sheaf over $\operatorname{Hilb}_{C / Y}^{n}$. Moreover, this section is always nonzero over a geometric point of $Y$ because curves over an algebraically closed field always have $n$ distinct rational points. By Proposition 1.1 we can conclude that $Z$ is flat over $Y$. For the remaining part of the statement we may assume $Y=\operatorname{Spec} k$, where $k$ is an algebraically closed field.

Let $C^{\prime}$ be a nonempty smooth open subset of a projective and integral curve over $k$ (we will reduce to a calculation on an open subset of a plane curve). Given indices $i \neq j \leqslant n$, we denote by $\Delta_{i, j}\left(C^{\prime n}\right)$ the effective Cartier divisor of $C^{\prime n}$ given by

$$
\Delta_{i, j}\left(C^{\prime n}\right)=\left\{\left(p_{1}, \ldots, p_{n}\right) \in C^{\prime n} \mid p_{i}=p_{j}\right\}
$$

Let $\mathcal{H}\left(C^{\prime n+1}\right) \subseteq C^{\prime n+1}$ be the zero locus of $1 \in \mathcal{O}_{C^{\prime n+1}}\left(\Delta_{1, n+1}\left(C^{\prime n+1}\right)+\cdots+\Delta_{n, n+1}\left(C^{\prime n+1}\right)\right)$. Notice that if $C^{\prime \prime} \subseteq C^{\prime}$ is a nonempty open subset, then $\mathcal{H}\left(C^{\prime \prime n+1}\right) \longrightarrow C^{\prime \prime n}$ is the restriction of the map $\mathcal{H}\left(C^{\prime n+1}\right) \longrightarrow C^{\prime n}$. Indeed, by definition we have

$$
\forall i \quad \Delta_{i, n+1}\left(C^{\prime n+1}\right) \cap\left(C^{\prime \prime n} \times C^{\prime}\right)=\Delta_{i, n+1}\left(C^{\prime \prime n+1}\right) \Longrightarrow \mathcal{H}\left(C^{\prime n+1}\right) \cap\left(C^{\prime \prime n} \times C^{\prime}\right)=\mathcal{H}\left(C^{\prime \prime n+1}\right) .
$$

We claim that $\mathcal{H}\left(C^{\prime n+1}\right) \longrightarrow C^{\prime n}$ is a degree $n$ cover. Indeed, by the discussion above, we can assume that $C^{\prime}$ is projective: in this case the map $\mathcal{H}\left(C^{\prime n+1}\right) \longrightarrow C^{\prime n}$ is flat thanks to Proposition 1.1, proper, quasi-finite and generically étale of degree $n$. Set $Z^{\prime}$ for the discriminant locus of $\mathcal{H}\left(C^{\prime n+1}\right)$ in $C^{\prime n}$ and $P \in C^{\prime n}$ for the generic point of $\Delta_{1,2}\left(C^{\prime n}\right)$, which lies in $Z^{\prime}$. We will show that $1\left(\mathcal{O}_{Z^{\prime}, P}\right)=2$. We first show how to conclude the proof from this fact. When $C^{\prime}=C$ the cover $\mathcal{H}\left(C^{n+1}\right)$ induces a map

$$
f: C^{n} \longrightarrow \operatorname{Hilb}_{C / k}^{n}
$$

which factors through an isomorphism $S^{n} C \simeq \mathrm{Hilb}_{C / k}^{n}$. In particular, $f$ is a cover and $Z^{\prime}=$ $f^{-1}(Z)$. Moreover, topologically, $Z$ equals $f\left(\Delta_{1,2}\left(C^{n}\right)\right)$ and coincides with the ramification locus of $f$. In particular $Z$ is nonempty and irreducible. Since Hilb $C_{/ k}^{n}$ is smooth, to see that $Z$ is reduced we have to prove that $1\left(\mathcal{O}_{Z, f(P)}\right)=1$. By Remark 3.8 and our assumption we have

$$
2=\mathrm{l}\left(\mathcal{O}_{Z^{\prime}, P}\right)=\mathrm{l}\left(\mathcal{O}_{Z^{\prime}, P} / m_{f(P)} \mathcal{O}_{Z^{\prime}, P}\right) \mathrm{l}\left(\mathcal{O}_{Z, f(P)}\right) \geqslant \mathrm{l}\left(\mathcal{O}_{Z, f(P)}\right)
$$




\section{Flavia Poma, Mattia Talpo and Fabio Tonini}

and we can exclude the equality because otherwise $f$ would be unramified in $P$, and by the transitivity of $S_{n}$, also over $f(P)$, which is not the case.

We have to prove that $1\left(\mathcal{O}_{Z^{\prime}, P}\right)=2$. Thanks to the discussion above we see that the ring $\mathcal{O}_{Z^{\prime}, P}$ (and therefore the number $1\left(\mathcal{O}_{Z^{\prime}, P}\right)$ ) does not change if we take an open subset of $C^{\prime}$. In particular, projecting in $\mathbb{P}_{k}^{2}$, we may assume that $C^{\prime}$ is of the form

$$
C^{\prime}=\operatorname{Spec} A \quad \text { where } \quad A=k[x, y]_{g} /(f)
$$

and $f, g$ are polynomials. We have $C^{\prime n}=\operatorname{Spec} B$, where

$$
B=A^{\otimes n}=k\left[x_{1}, y_{1}, \ldots, x_{n}, y_{n}\right]_{g\left(x_{1}, y_{1}\right) \cdots g\left(x_{n}, y_{n}\right)} /\left(f\left(x_{1}, y_{1}\right), \ldots, f\left(x_{n}, y_{n}\right)\right)
$$

and $P=\left(x_{2}-x_{1}, y_{2}-y_{1}\right)$. By definition $\mathcal{H}\left(C^{\prime n+1}\right)$ is the spectrum of the $B$-algebra

$$
D=B[\alpha, \beta]_{g(\alpha, \beta)} /\left(f(\alpha, \beta), \prod_{j} I_{j}\right) \quad \text { where } \quad I_{j}=\left(\alpha-x_{j}, \beta-y_{j}\right) .
$$

We have to compute the discriminant locus of the cover $D_{P}=D \otimes B_{P}$ over $B_{P}$ and show that it has length two. Since $C^{\prime}$ is smooth, $B_{P}$ is a discrete valuation ring (DVR). In particular, we may assume that $P$ is generated by $x_{1}-x_{2}$ in $B_{P}$, so that $x_{1}-x_{2} \mid y_{1}-y_{2}$ in $B_{P}$. Notice that $\left(\alpha-x_{1}\right) \cdots\left(\alpha-x_{l}\right),\left(\alpha-x_{l+1}\right) \in I_{1} \cdots I_{l}+I_{l+1} \Longrightarrow\left(x_{l+1}-x_{1}\right) \cdots\left(x_{l+1}-x_{l}\right) \in I_{1} \cdots I_{l}+I_{l+1}$ in $B[\alpha, \beta]_{g(\alpha, \beta)} /(f(\alpha, \beta))$. Looking at the quotient $B / P=A^{\otimes(n-1)}$, we see that the product $\left(x_{l+1}-x_{1}\right) \cdots\left(x_{l+1}-x_{l}\right)$ is not an element of $P$ for $l>1$. So $I_{1} \cdots I_{l}+I_{l+1}$ is the trivial ideal in $D_{P}$ and applying the Chinese remainder theorem inductively it follows that

$$
D_{P}=\left(D_{P} / I_{1} I_{2}\right) \times D_{P} / I_{3} \times \cdots \times D_{P} / I_{n} .
$$

Since $D_{P} / I_{l} \simeq B_{P}$, the discriminant locus of $D_{P}$ over $B_{P}$ coincides with the discriminant locus of $E=\left(D_{P} / I_{1} I_{2}\right)$ over $B_{P}$, which is a cover of degree two, since $D_{P}$ is a cover of degree $n$ of $B_{P}$. From $I_{1} I_{2}=0$ in $E$ we get relations

$$
\alpha^{2}=\left(x_{1}+x_{2}\right) \alpha-x_{1} x_{2} \quad \text { and } \quad\left(x_{1}-x_{2}\right) \beta=\left(y_{1}-y_{2}\right) \alpha+x_{1} y_{2}-y_{1} x_{2} .
$$

Since $x_{1}-x_{2}$ divides $y_{1}-y_{2}$ in $B_{P}, E$ is generated by $1, \alpha$ as $B_{P}$-module. Moreover, since $E$ is a free $B_{P}$-module of rank two, $1, \alpha$ is also a $B_{P}$-basis of $E$. Finally, a direct computation shows that

$$
\operatorname{tr}(\alpha)=x_{1}+x_{2}, \operatorname{tr}\left(\alpha^{2}\right)=\left(x_{1}+x_{2}\right)^{2}-2 x_{1} x_{2}, \quad \operatorname{det}\left(\begin{array}{cc}
\operatorname{tr}(1) & \operatorname{tr}(\alpha) \\
\operatorname{tr}(\alpha) & \operatorname{tr}\left(\alpha^{2}\right)
\end{array}\right)=\left(x_{1}-x_{2}\right)^{2},
$$

where $\operatorname{tr}=\operatorname{tr}_{E / B_{P}}$. The last determinant is the discriminant section of the cover $E / B_{P}$ and therefore its discriminant locus has length two, as claimed.

Proof of Theorem 3.2, first sentence and (ii). By Proposition 3.7 we have $\mathcal{Z}_{d, g, n}=\emptyset \Longleftrightarrow$ $\mathcal{Z}_{d n, g, 1}=\emptyset$ and, by Theorem 3.9, this happens if and only if $d n=1$. So assume $d n>1$. Again by Proposition 3.7 we may assume $n=1$. The result then follows from Proposition 3.4 and Theorem 3.9.

We now deal with the problem of reducibility of the stacks $\mathcal{Z}_{d, g, n}$.

Lemma 3.10. Let $k$ be an algebraically closed field and let $C$ be a genus $g$ curve over $k$. If $\mathcal{Q}$ is an invertible sheaf of degree $d$ with $d>2 g-2$ and $q \in C(k)$ then the map

$$
\mathrm{H}^{0}(\mathcal{Q}) \longrightarrow \mathcal{Q} \otimes\left(\mathcal{O}_{C, q} / m_{q}^{2} \mathcal{O}_{C, q}\right)
$$




\section{StACKS OF UNIFORM CYCLIC COVERS OF CURVES AND THEIR PICARD GROUPS}

where $m_{q}$ is the maximal ideal of $\mathcal{O}_{C, q}$, has cokernel $\mathrm{H}^{0}\left(\mathcal{Q}^{-1} \otimes \mathcal{O}_{C}(2 q) \otimes \omega_{C}\right)$; it is surjective if $d>2 g$.

Proof. Consider the exact sequence

$$
0 \longrightarrow \mathcal{Q} \otimes \mathcal{O}_{C}(-2 q) \longrightarrow \mathcal{Q} \longrightarrow \mathcal{Q} \otimes\left(\mathcal{O}_{C, q} / m_{q}^{2} \mathcal{O}_{C, q}\right) \longrightarrow 0
$$

Since $\mathrm{H}^{1}(\mathcal{Q}) \simeq \mathrm{H}^{0}\left(\mathcal{Q}^{-1} \otimes \omega_{C}\right)=0$ by degree reasons, applying $\mathrm{H}^{0}$ we get an exact sequence

$$
\mathrm{H}^{0}(\mathcal{Q}) \longrightarrow \mathcal{Q} \otimes\left(\mathcal{O}_{C, q} / m_{q}^{2} \mathcal{O}_{C, q}\right) \longrightarrow \mathrm{H}^{1}\left(\mathcal{Q} \otimes \mathcal{O}_{C}(-2 q)\right) \simeq \mathrm{H}^{0}\left(\mathcal{Q}^{-1} \otimes \mathcal{O}_{C}(2 q) \otimes \omega_{C}\right) \longrightarrow 0 .
$$

Finally, if $d>2 g$, then $\mathrm{H}^{0}\left(\mathcal{Q}^{-1} \otimes \mathcal{O}_{C}(2 q) \otimes \omega_{C}\right)=0$, again by degree reasons.

Proof of Theorem 3.2(iii). The case $n=1$ follows from Proposition 3.4 and Theorem 3.9. So we focus on the case $n d>2 g-2$. We remark that the proof is a bit simpler if we have the stronger inequality $n d>2 g$, and the intermediate cases require a finer inspection.

Set $\mathcal{V}=\mathcal{V}_{d, g, n}$ and $\mathcal{U}=\mathcal{U}_{d, g, n}$. By Remark $3.3 \mathcal{V}$ is a vector bundle over $\mathcal{J}_{a c_{d, g}}$ and $\mathcal{U}$ is the complement of the zero section. Consider the diagram

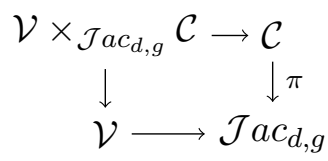

where $\mathcal{C}$ is the universal curve over $\mathcal{J} a c_{d, g}$. Denote by $\mathcal{L}$ the universal invertible sheaf over $\mathcal{C}$, so that $\mathcal{V}$ corresponds to $\pi_{*} \mathcal{L}^{n}$. By [Gro67, Section 16.7] there exists a locally free sheaf $\mathcal{F}$ on $\mathcal{C}$, the 2nd bundle of principal parts of $\mathcal{L}^{n}$, and a map $\alpha: \pi^{*} \pi_{*}\left(\mathcal{L}^{n}\right) \longrightarrow \mathcal{F}$ such that for all algebraically closed fields $k$ and triples $(C, \mathcal{Q}, q) \in \mathcal{C}(k)$, where $q \in C(k)$, we have $\mathcal{F} \otimes k \simeq \mathcal{Q}^{n} \otimes \mathcal{O}_{C, q} / m_{q}^{2}$, where $m_{q}$ is the maximal ideal of $\mathcal{O}_{C, q}$ and

$$
\alpha \otimes k: \mathrm{H}^{0}\left(\mathcal{Q}^{n}\right) \simeq \pi^{*} \pi_{*}\left(\mathcal{L}^{n}\right) \otimes k \longrightarrow \mathcal{F} \otimes k \simeq \mathcal{Q}^{n} \otimes \mathcal{O}_{C, q} / m_{q}^{2}
$$

is the restriction. If $n d>2 g$, by Lemma 3.10 we can conclude that $\alpha$ is surjective. In this case in what follows set $\mathcal{W}=\mathcal{J} a c_{d, g}$ and $\mathcal{Y}=\emptyset$. If $n d \leqslant 2 g$, we want to find an open substack $\mathcal{W}$ of $\mathcal{J} a c_{d, g}$ over which $\alpha$ is surjective. If $d n=2 g$ and $g \geqslant 3$, consider the map

$$
\beta: \mathcal{M}_{g, 1} \longrightarrow \mathcal{J}_{a c_{d n, g}},(C \longrightarrow S, \sigma) \longmapsto\left(C, \mathcal{O}_{C}(2 \sigma) \otimes \omega_{C / S}\right)
$$

while if $d n=2 g-1$ and $g \geqslant 4$, consider the map

$$
\beta: \mathcal{M}_{g, 1} \times_{\mathcal{M}_{g}} \mathcal{M}_{g, 1} \longrightarrow \mathcal{J} a c_{d n, g},(C \longrightarrow S, \sigma, \tau) \longmapsto\left(C, \mathcal{O}_{C}(2 \sigma-\tau) \otimes \omega_{C / S}\right) .
$$

In both cases denote by $\mathcal{Y}^{\prime}$ the closed substack of $\mathcal{J} a c_{d n, g}$ whose topological space is the closure of the image of $\beta$, set $\mathcal{Y}=n^{-1}\left(\mathcal{Y}^{\prime}\right)$ where $n: \mathcal{J} a c_{d, g} \longrightarrow \mathcal{J} a c_{d n, g}$ is the elevation to the $n$-th power and $\mathcal{W}=\mathcal{J} a c_{d, g}-\mathcal{Y}$.

We will denote by $-\mathcal{W}$ the restriction to $\mathcal{W}$. We want to prove that $\alpha_{\mathcal{W}}$ is surjective and we can check this on the geometric points of $\mathcal{C}_{\mathcal{W}}$. Given $(C, \mathcal{Q}, q) \in \mathcal{C}_{\mathcal{W}}(k)$, where $k$ is an algebraically closed field, by Lemma 3.10, the cokernel of $\alpha \otimes k$ is $\mathrm{H}^{0}(\mathcal{T})$ where $\mathcal{T}=\mathcal{Q}^{-n} \otimes \mathcal{O}_{C}(2 q) \otimes \omega_{C}$. Notice that $\mathcal{T}$ has degree $-d n+2 g$. Assume by contradiction $\mathrm{H}^{0}(\mathcal{T}) \neq 0$. If $d n=2 g$, then $\mathcal{T} \simeq \mathcal{O}_{C}$ and therefore $n(C, \mathcal{Q}) \simeq \beta(C, q)$, which is not the case by construction of $\mathcal{W}$. Finally, if $d n=2 g-1$, then by Lemma 1.11 there exists $q^{\prime} \in C$ such that $\mathcal{T} \simeq \mathcal{O}_{C}\left(q^{\prime}\right)$, which means $n(C, \mathcal{Q}) \simeq \beta\left(C, q, q^{\prime}\right)$, which is again not possible by construction.

We want to prove that $\left(\mathcal{Z}_{d, g, n}\right)_{\mathcal{W}}$ is irreducible. Since $\mathcal{J} a c_{d, g}$ and $\mathcal{C}$ are integral and the vector bundle associated with $\pi^{*} \pi_{*}\left(\mathcal{L}^{n}\right)$ is $\mathcal{V} \times{ }_{\mathcal{J} a c_{d, g}} \mathcal{C}$, the kernel $\mathcal{Z}$ of $\alpha_{\mathcal{W}}$ is an integral closed substack of 


\section{Flavia Poma, Mattia Talpo and Fabio Tonini}

$\left(\mathcal{V} \times{\mathcal{J} a c_{d, g}} \mathcal{C}\right)_{\mathcal{W}}$. Let $\widehat{\mathcal{Z}}$ be the image of $\mathcal{Z}$ via the projection $\left(\mathcal{V} \times{\mathcal{J} a c_{d, g}} \mathcal{C}\right)_{\mathcal{W}} \longrightarrow \mathcal{V}_{\mathcal{W}}$. We want to prove that $\left(\mathcal{Z}_{d, g, n}\right)_{\mathcal{W}}=\widehat{\mathcal{Z}} \cap \mathcal{U}$ topologically. This will imply the irreducibility of $\left(\mathcal{Z}_{d, g, n}\right)_{\mathcal{W}}$.

In what follows $k$ will be an arbitrary algebraically closed field. The objects of $\mathcal{Z}(k)$ are tuples $(C, \mathcal{Q}, q, s) \in\left(\mathcal{V} \times \mathcal{J}_{a c_{d, g}} \mathcal{C}\right)_{\mathcal{W}}(k)$, where $q \in C(k)$ and $s \in \mathcal{Q}^{n}$ such that $v_{q}(s) \geqslant 2$, where $v_{q}$ denotes the valuation in $q$. Thus the objects of $(\widehat{\mathcal{Z}} \cap \mathcal{U})(k)$ are triples $(C, \mathcal{Q}, s) \in \mathcal{U}_{\mathcal{W}}(k)$ for which there exists $q \in C(k)$ such that $v_{q}(s) \geqslant 2$. The result then follows from the following remark. If $(C, Q, s) \in \mathcal{U}(k)$, then the zero locus $Z(s) \subseteq C$ of $s$ is étale over $k$ if and only if for all $q \in C(k)$ the ring $\mathcal{O}_{C, q} / s_{q}\left(\simeq \mathcal{O}_{Z(s), q}\right)$ is either 0 or $k$, that is, if and only if for all $q \in C(k)$ we have $v_{q}(s)<2$.

If $d n>2 g$, so that $\mathcal{W}=\mathcal{J} a c_{d, g}$, then $\left(\mathcal{Z}_{d, g, n}\right)_{\mathcal{W}}=\mathcal{Z}_{d, g, n}$ is irreducible as required. So assume $2 g-2<d n \leqslant 2 g$ and $g \geqslant 2$. Denote by $f: \mathcal{U} \longrightarrow \mathcal{J} a c_{d, g}$ the structure map. Topologically we have

$$
\mathcal{Z}_{d, g, n} \subseteq \overline{\left(\mathcal{Z}_{d, g, n}\right) \mathcal{W}} \cup f^{-1}(\mathcal{Y}),
$$

where the closure is taken inside $\mathcal{U}$. If $\mathcal{Z}_{d, g, n}=\mathcal{U}$ (which a posteriori will not be the case), there is nothing to prove. Otherwise, since $\mathcal{Z}_{d, g, n} \neq \emptyset$, by Corollary 1.7 the equality $\mathcal{Z}_{d, g, n}=\overline{\left(\mathcal{Z}_{d, g, n}\right)_{\mathcal{W}}}$ follows from $\operatorname{dim} \mathcal{U}-\operatorname{dim} f^{-1}(\mathcal{Y}) \geqslant 2$, which we are going to prove. Thanks to Proposition 3.6 the map $\mathcal{U} \stackrel{f}{\longrightarrow} \mathcal{J} a c_{d, g} \stackrel{n}{\longrightarrow} \mathcal{J} a c_{d n, g}$ has constant relative dimension. Moreover, $n^{-1}\left(\mathcal{Y}^{\prime}\right)=\mathcal{Y}$ and, by Proposition 1.6, we get

$$
\operatorname{dim} \mathcal{U}-\operatorname{dim} f^{-1}(\mathcal{Y})=\operatorname{dim} \mathcal{J} a c_{d n, g}-\operatorname{dim} \mathcal{Y}^{\prime} .
$$

If $d n=2 g$, set $\mathcal{M}=\mathcal{M}_{g, 1}$, while if $d n=2 g-1$, set $\mathcal{M}=\mathcal{M}_{g, 1} \times_{\mathcal{M}_{g}} \mathcal{M}_{g, 1}$. Let us also write $\delta: \mathcal{J} a c_{d n, g} \longrightarrow \underline{\operatorname{Pic}}_{\mathcal{M}_{g, 1} / \mathcal{M}_{g}}^{d n}$ for the natural map, and $\gamma=\delta \circ \beta$. Since $\mathcal{M}$ and $\underline{\operatorname{Pic}}_{\mathcal{M}_{g, 1} / \mathcal{M}_{g}}^{d n}$ are proper over $\mathcal{M}_{g}$ and are Deligne-Mumford stacks, we can conclude that $\gamma(\mathcal{M})$ is closed and, by Corollary 1.8, that

$$
\operatorname{dim} \gamma(\mathcal{M}) \leqslant \operatorname{dim} \mathcal{M}
$$

Since $\delta: \mathcal{J} a c_{d n, g} \longrightarrow \underline{\operatorname{Pic}}_{\mathcal{M}_{g, 1} / \mathcal{M}_{g}}^{d n}$ is a $\mathbb{G}_{m}$-gerbe, we also have $\delta^{-1}(\gamma(\mathcal{M}))=\mathcal{Y}^{\prime}$, so that, by Proposition 1.6,

$$
\operatorname{dim} \mathcal{J} a c_{d n, g}-\operatorname{dim} \mathcal{Y}^{\prime}=\operatorname{dim} \underline{\operatorname{Pic}}_{\mathcal{M}_{g, 1} / \mathcal{M}_{g}}-\operatorname{dim} \gamma(\mathcal{M}) \geqslant g+\operatorname{dim} \mathcal{M}_{g}-\operatorname{dim} \mathcal{M} .
$$

Since $\operatorname{dim} \mathcal{M}=1+\operatorname{dim} \mathcal{M}_{g}$ if $d n=2 g$ and $\operatorname{dim} \mathcal{M}=2+\operatorname{dim} \mathcal{M}_{g}$ if $d n=2 g-1$, we get the desired formula.

When $d n \leqslant 2 g-2$ it is not clear whether $\mathcal{Z}_{d, g, n}$ is irreducible or not. The main technical issue here is that $\mathcal{V}_{d, g, n} \longrightarrow \mathcal{J} a c_{d, g}$ is no longer a vector bundle and $\mathcal{U}_{d, g, n} \longrightarrow \mathcal{J} a c_{d, g}$ may not be surjective.

When $d n>2 g-2$, we have shown above that the stack $\mathcal{Z}_{d, g, n}$ is irreducible except in the following cases: $g=1$ and $(d, n)=(1,2) ; g=2$ and $(d, n) \in\{(1,4),(2,2),(1,3)\} ; g=3$ and $(d, n)=(1,5)$. In the remaining part of the section we work out the case $g=1, d=1$ and $n=2$ (so that $d n=2 g$ ). As claimed in Theorem 3.2(iv), we will see that $\mathcal{Z}_{1,1,2}$ is reducible. In the other cases just listed, it is again not clear whether $\mathcal{Z}_{d, g, n}$ is irreducible or not.

Lemma 3.11. Assume $p \neq 2$. Let $\mathcal{C} \longrightarrow \mathcal{S}$ be a curve over an algebraic stack with a section $\tau$ and denote by $\mathcal{W}$ the zero locus of $1 \in \mathcal{O}_{\mathcal{C}}(2 \tau)$ in $\mathcal{C}$. Then $\rho: \mathcal{W} \longrightarrow \mathcal{S}$ is a degree two cover. Moreover, $\tau$ factors through $\mathcal{W}$ and the induced map $\rho_{*} \mathcal{O}_{\mathcal{W}} \longrightarrow \mathcal{O}_{\mathcal{S}}$ is $\operatorname{tr}_{\rho_{*}} \mathcal{O}_{\mathcal{W}} / 2$, where tr denotes the trace map; its kernel is a square zero ideal.

Proof. The map $\rho: \mathcal{W} \longrightarrow \mathcal{S}$ is a degree two cover thanks to Lemma 1.11 and the section $\mathcal{S} \longrightarrow \mathcal{C}$ factors through $\mathcal{W}$ by definition of this last space. Denote by $\psi: \rho_{*} \mathcal{O}_{\mathcal{W}} \longrightarrow \mathcal{O}_{\mathcal{S}}$ the induced map. 


\section{StACKS OF UNIFORM CYCLIC COVERS OF CURVES AND THEIR PICARD GROUPS}

Since $\mathcal{M}_{g, 1}$ is reduced, we may assume $\mathcal{S}=\operatorname{Spec} k$, where $k$ is an algebraically closed field. In this case the result follows because $\rho_{*} \mathcal{O}_{\mathcal{W}}=k[x] /\left(x^{2}\right)$.

Proof of Theorem 3.2(iv). If we denote by $\overline{\mathcal{E}}$ the universal curve over $\mathcal{M}_{1,1}$, we are going to show that there is a $\mu_{2}$-gerbe $\mathcal{Z}_{1,1,2} \longrightarrow \overline{\mathcal{E}}[2]$. Since this last group is a disjoint union of two irreducible components and gerbes are geometrically irreducible, we will conclude that $\mathcal{Z}_{1,1,2}$ is also a disjoint union of two irreducible components. We will then study the component over the zero section of $\overline{\mathcal{E}}[2]$ and represent it as zero locus of a section of an invertible sheaf.

Set $\mathcal{E}=\mathcal{C}$ for the universal curve of $\mathcal{U}_{1,1,2}$. The canonical cover $\mathcal{H}_{1,1,2} \longrightarrow \mathcal{U}_{1,1,2}$ of $\mathcal{U}_{1,1,2}$ has degree two. By standard theory of double covers it is given by an invertible sheaf $\mathcal{T}$ over $\mathcal{U}_{1,1,2}$ and a section $r \in \mathcal{T}^{2}$, so that $\mathcal{H}_{1,1,2}=\operatorname{Spec} \mathscr{A}$ where $\mathscr{A}=\mathcal{O}_{\mathcal{U}_{1,1,2}} \oplus \mathcal{T}^{-1}$. By an easy local computation, the discriminant section of the canonical cover coincides, up to an invertible element, with $r$ and therefore $\mathcal{Z}_{1,1,2}$ is the zero locus of $r$. Set $\mathcal{Z}=\mathcal{Z}_{1,1,2}$. We will use the symbol $-\mathcal{Z}$ for base changes along $\mathcal{Z} \longrightarrow \mathcal{U}_{1,1,2}$. For instance, $\mathcal{E}_{\mathcal{Z}}$ is the universal curve over $\mathcal{Z}$ with universal invertible sheaf $\mathcal{L}_{\mathcal{Z}}$. Since $r_{\mathcal{Z}}=0$, we have $\left(\mathcal{T}_{\mathcal{Z}}^{-1}\right)^{2}=0$ in $\mathscr{A}_{\mathcal{Z}}$. Therefore the projection $\mathscr{A}_{\mathcal{Z}} \longrightarrow \mathcal{O}_{\mathcal{Z}}$ is a ring homomorphism and thus induces a section $\mathcal{Z} \longrightarrow \mathcal{H}_{\mathcal{Z}}$ and therefore a section $\tau: \mathcal{Z} \longrightarrow \mathcal{E}_{\mathcal{Z}}$. This yields a unique map $\mathcal{O}_{\mathcal{E}_{\mathcal{Z}}}(\tau) \longrightarrow \mathcal{L}_{\mathcal{Z}}^{2}$ that sends 1 to $s$ and therefore a section $s^{\prime} \in \mathcal{N}=\mathcal{L}_{\mathcal{Z}}^{2} \otimes \mathcal{O}_{\mathcal{E}_{\mathcal{Z}}}(-\tau)$. Since $s^{\prime}$ is nonzero on the geometric fibers of $\pi_{\mathcal{Z}}: \mathcal{E}_{\mathcal{Z}} \longrightarrow \mathcal{Z}$, by Lemma 1.11 there exist another section $\tau^{\prime}: \mathcal{Z} \longrightarrow \mathcal{E}_{\mathcal{Z}}$ and an isomorphism $\mathcal{O}_{\mathcal{E}_{\mathcal{Z}}}\left(\tau^{\prime}\right) \simeq \mathcal{N}$ sending 1 to $s^{\prime}$. Since the cover $\left(\mathcal{H}_{1,1,2}\right)_{\mathcal{Z}} \longrightarrow \mathcal{Z}$ is topologically a homeomorphism, the sections $\tau$ and $\tau^{\prime}$ coincide on the geometric fibers of $\pi_{\mathcal{Z}}$. Since $\mathcal{Z}$ is reduced thanks to Theorem 3.2(ii), we can conclude that $\tau=\tau^{\prime}$. Moreover, the induced isomorphism $\mathcal{O}_{\mathcal{E}_{\mathcal{Z}}}(2 \tau) \longrightarrow \mathcal{L}_{\mathcal{Z}}^{2}$ sends 1 to $s$.

Define $\mathcal{Z}^{\prime}$ as the stack of tuples $(E, \mathcal{G}, \tilde{\tau}, \lambda)$ where $E$ is a genus one curve over $S, \mathcal{G}$ is a degree zero invertible sheaf over $E, \tilde{\tau}$ is a section of $E$ and $\lambda: \mathcal{G}^{2} \longrightarrow \mathcal{O}_{E}$ is an isomorphism. The discussion above shows that we have a map $\mathcal{Z} \longrightarrow \mathcal{Z}^{\prime}$ which sends $(E, \mathcal{Q}, s)$ to $(E, \mathcal{G}, \tilde{\tau}, \lambda)$, where $\tilde{\tau}$ is induced by the section $\tau: \mathcal{Z} \longrightarrow \mathcal{E}_{\mathcal{Z}}, \mathcal{G}=\mathcal{Q} \otimes \mathcal{O}_{E}(-\tilde{\tau})$ and the isomorphism $\lambda$ is the base change of the isomorphism $\mathcal{O}_{\mathcal{E}_{\mathcal{Z}}}(2 \tau) \longrightarrow \mathcal{L}_{\mathcal{Z}}^{2}$. Conversely, we can define a map $\mathcal{Z}^{\prime} \longrightarrow \mathcal{Z}$ by sending $(E, \mathcal{G}, \tilde{\tau}, \lambda)$ to $(E, \mathcal{Q}, s)$ where $\mathcal{Q}=\mathcal{G} \otimes \mathcal{O}_{E}(\tilde{\tau})$ and $s$ is the image of 1 under the isomorphism

$$
\mathcal{O}_{E}(2 \tilde{\tau}) \simeq \mathcal{G}^{2} \otimes \mathcal{O}_{E}(2 \tilde{\tau}) \simeq \mathcal{Q}^{2}
$$

By Lemma 3.11 we see that the last functor is well defined and that the composition $\mathcal{Z}^{\prime} \longrightarrow$ $\mathcal{Z} \longrightarrow \mathcal{Z}^{\prime}$ is equivalent to the identity. Conversely, the composition $\mathcal{Z} \longrightarrow \mathcal{Z}^{\prime} \longrightarrow \mathcal{Z}$ is equivalent to the identity because the map $\mathcal{O}_{\mathcal{E}_{\mathcal{Z}}}(2 \tau) \longrightarrow \mathcal{L}_{\mathcal{Z}}^{2}$ sends 1 to $s$.

We define the map

$$
\begin{array}{cc}
\mathcal{Z}^{\prime} & p \\
(E, \mathcal{G}, \tilde{\mathcal{E}}[2] & \longrightarrow(E, \tilde{\tau},[\mathcal{G}]) .
\end{array}
$$

where we identify $\overline{\mathcal{E}}$ with $\underline{\operatorname{Pic}_{\overline{\mathcal{E}}}^{0}}{ }_{/ \mathcal{M}_{1,1}}^{0}$ (see Lemma 2.11 ), which is easily seen to be a $\mu_{2}$-gerbe.

Now we prove that $\overline{\mathcal{E}}[2]$ is a disjoint union of two irreducible components, one being the zero section $\mathcal{M}_{1,1} \longrightarrow \overline{\mathcal{E}}[2]$. First of all, since $\overline{\mathcal{E}}[2]$ is étale, the zero section is a connected component of $\overline{\mathcal{E}}[2]$. So we need to prove that the complement $H$ is irreducible as well. But $H \longrightarrow \mathcal{M}_{1,1}$ is an étale degree three cover and thus, if $H$ is not connected (and therefore irreducible, being smooth), it should have a section, which is not the case thanks to Lemma 2.18 and Proposition 2.19.

Since $p: \mathcal{Z}^{\prime} \longrightarrow \overline{\mathcal{E}}[2]$ is a $\mu_{2}$-gerbe and thus has irreducible fibers, we can conclude that $\mathcal{Z}^{\prime} \simeq \mathcal{Z}$ is a disjoint union of two irreducible substack, one of which is $\mathcal{Z}_{0}=p^{-1}\left(\mathcal{M}_{1,1}\right)$. We identify $\mathcal{Z}^{\prime}$ 
with $\mathcal{Z}$ and will write $\mathcal{Z}_{0}$ for the zero locus of a section of the invertible sheaf in the statement. So with an object $(E, \mathcal{Q}, s) \in \mathcal{Z}$ are associated a section $\tilde{\tau}$ of $E$, base change of $\tau: \mathcal{Z} \longrightarrow \mathcal{E}_{\mathcal{Z}}$ and an isomorphism $\mathcal{O}_{E}(2 \tilde{\tau}) \longrightarrow \mathcal{Q}^{2}$ sending 1 to $s$, base change of the isomorphism $\mathcal{O}_{\mathcal{E}_{\mathcal{Z}}}(2 \tau) \longrightarrow \mathcal{L}_{\mathcal{Z}}^{2}$. The objects of $\mathcal{Z}_{0}$ are the triples $(E, \mathcal{Q}, s) \in \mathcal{Z}(S)$ such that $\mathcal{Q}$ and $\mathcal{O}_{E}(\tilde{\tau})$ differ by an invertible sheaf from the base $S$, that is $[\mathcal{Q}]=\left[\mathcal{O}_{E}(\tilde{\tau})\right]$ in $\underline{\operatorname{Pic}}_{E / S}^{1}$. Since $\mathcal{L}$ is an invertible sheaf of degree one on $\mathcal{E}, \mathcal{R}=\pi_{*} \mathcal{L}$ is an invertible sheaf by Lemma 1.10 and there exists a unique section $\sigma: \mathcal{U}_{1,1,2} \longrightarrow \mathcal{E}$ with an isomorphism $\mathcal{L} \simeq \mathcal{O}_{\mathcal{E}}(\sigma) \otimes \pi^{*} \mathcal{R}$ by Lemma 2.11.

Let $\mathcal{W}$ be the zero locus in $\mathcal{E}$ of the section $1 \in \mathcal{O}_{\mathcal{E}}(2 \sigma)$. The induced map $\mathcal{W} \longrightarrow \mathcal{U}_{1,1,2}$ is a degree two cover by Lemma 3.11. Tensoring the exact sequence defining $\mathcal{O}_{\mathcal{W}}$ by $\mathcal{L}^{2}$, we get an exact sequence

$$
0 \longrightarrow \pi^{*} \mathcal{R}^{2} \stackrel{\alpha}{\longrightarrow} \pi^{*} \mathcal{R}^{2} \otimes \mathcal{O}_{E}(2 \sigma) \simeq \mathcal{L}^{2} \longrightarrow \mathcal{O}_{W} \otimes \mathcal{L}^{2} \longrightarrow 0,
$$

where $\alpha(x)=x \otimes 1$. Applying $\pi_{*}$ and taking into account Lemma 1.10 we get an exact sequence

$$
0 \longrightarrow \mathcal{R}^{2} \stackrel{\pi_{*} \alpha}{\longrightarrow} \pi_{*} \mathcal{L}^{2} \longrightarrow \pi_{*}\left(\mathcal{O}_{W} \otimes \mathcal{L}^{2}\right) \longrightarrow \mathrm{R}^{1} \pi_{*}\left(\pi^{*} \mathcal{R}^{2}\right) \longrightarrow 0
$$

of locally free sheaves on $\mathcal{U}_{1,1,2}$. Note that the exact sequence on $\mathcal{E}$ satisfies base change for $\pi_{*}$. Set $\mathcal{N}=\operatorname{Coker}\left(\pi_{*} \alpha\right)$. This is an invertible sheaf. Applying the determinant we see that it coincides with the invertible sheaf in the statement. The section $s \in \pi_{*} \mathcal{L}^{2}$ induces a section $t \in \mathcal{N}$ and we claim that its zero locus is exactly $\mathcal{Z}_{0}$. This will conclude the proof. Let $\chi=(E \stackrel{f}{\longrightarrow} S, \mathcal{Q}, s) \in$ $\mathcal{U}_{1,1,2}$. We will denote by $-_{\chi}$ the base change along the corresponding map $S \longrightarrow \mathcal{U}_{1,1,2}$. For instance, $\mathcal{L}_{\chi}=\mathcal{Q}, f=\pi_{\chi}$ and, with abuse of notation, $s_{\chi}=s$. We have that $t_{\chi}=0$ if and only if $s_{\chi} \in \operatorname{Im}\left(f_{*} \alpha_{\chi}\right) \subseteq f_{*} \mathcal{Q}^{2}$ if and only if $s \in \operatorname{Im}\left(\alpha_{\chi}\right) \subseteq \mathcal{Q}^{2}$. So $t_{\chi}=0$ if and only if the square of the isomorphism $\mathcal{Q} \simeq f^{*} \mathcal{R}_{\chi} \otimes \mathcal{O}_{E}\left(\sigma_{\chi}\right)$ sends $s$ to a section of the form $x \otimes 1$. We want to show that those are exactly the objects of $\mathcal{Z}_{0}$; that is, $t_{\chi}=0$ if and only if $\chi \in \mathcal{Z}_{0}$.

If $\chi \in \mathcal{Z}_{0}$, we have $[\mathcal{Q}]=\left[\mathcal{O}_{E}\left(\sigma_{\chi}\right)\right]=\left[\mathcal{O}_{E}(\tilde{\tau})\right]$ in $\underline{\operatorname{Pic}}_{E / S}^{1}$, which implies $\sigma_{\chi}=\tilde{\tau}$ by Lemma 2.11 . Moreover, we have an isomorphism $\mathcal{O}_{E}(2 \tilde{\tau}) \simeq \mathcal{Q}^{2}$ sending 1 to $s$. We can conclude, observing that all the isomorphisms $\mathcal{O}_{E}(2 \tilde{\tau}) \longrightarrow \mathcal{O}_{E}(2 \tilde{\tau}) \otimes f^{*} \mathcal{R}^{2}$ send 1 to a section of the form $1 \otimes x$.

Assume now $t_{\chi}=0$, so that $s \in \mathcal{Q}^{2}$ corresponds to a section $x \otimes 1 \in f^{*} \mathcal{R}_{\chi}^{2} \otimes \mathcal{O}_{E}\left(2 \sigma_{\chi}\right)$. If $x \in f^{*} \mathcal{R}_{\chi}^{2}$ does not generate this sheaf, then the zero locus of $s \in \mathcal{Q}^{2}$ inside $E$ cannot be a cover of $S$, because it will have nonzero dimensional fibers, contradicting the fact that $\chi \in \mathcal{U}_{1,1,2}$. So $f^{*} \mathcal{R}_{\chi}^{2} \simeq \mathcal{O}_{E}$, and the zero locus of $s$ in $E$ is the base change of $\mathcal{W} \subseteq \mathcal{E}$, the zero locus of $1 \in \mathcal{O}_{\mathcal{E}}(2 \sigma)$. Taking into account Lemma 3.11, this shows that $\chi \in \mathcal{Z}$. It also implies $\tilde{\tau}=\sigma$, so that $\left[\mathcal{Q} \otimes \mathcal{O}_{E}(-\tilde{\tau})\right]=0$ in $\underline{\operatorname{Pic}}_{E / S}^{0}$. This exactly means that $\chi \in \mathcal{Z}_{0}$, as required.

\section{Stacks of uniform cyclic covers and their Picard groups}

In this section we work over a field of characteristic $p \geqslant 0$ and fix a nonnegative integer $g$ and a positive integer $n$ with $n \geqslant 2$.

Definition 4.1. Let $Y$ be a scheme. A uniform cyclic cover of degree $n$ of $Y$ is a map $f: X \longrightarrow Y$ together with an action of $\mu_{n}$ on $X$ such that for all $q \in Y$ there exist an affine open neighborhood $U=\operatorname{Spec} R$ of $q$, an element $h \in R$ and a $\mu_{n}$-equivariant isomorphism of $U$-schemes $f^{-1}(U) \simeq$ Spec $R[x] /\left(x^{n}-h\right)$, where the right-hand side is given the action for which $\operatorname{deg} x=1$.

Uniform cyclic covers of degree $n$ form a stack that we denote by $\mathcal{U C}_{n}$.

Notice that uniform cyclic covers of degree $n$ are covers of degree $n$ and can be seen as a generalization of double covers when $p \neq 2$. The definition of uniform cyclic covers in [AV04] 


\section{StACKS OF UNIFORM CYCLIC COVERS OF CURVES AND THEIR PICARD GROUPS}

is slightly different from ours, because in Definition 4.1 we do not require that $h$ be a nonzero divisor. The reason is that this is automatic for uniform cyclic covers between schemes smooth on a common base and that, avoiding this restriction, uniform cyclic covers are stable by base change.

Definition 4.2. Let $h$ be a natural number. We denote by $\mathcal{B}_{h, g, n}$ the stack of triples $(D, C, f)$ where $D \longrightarrow S$ is a genus $h$ curve, $C \longrightarrow S$ is a genus $g$ curve and $f: D \longrightarrow C$ is a uniform cyclic cover of degree $n$.

We define the number $d(h, g, n)=2 \frac{h+n(1-g)-1}{n(n-1)}$, so that $h=1+n(g-1)+\frac{n(n-1)}{2} d(h, g, n)$.

The aim of this section is to describe $\mathcal{B}_{h, g, n}$ and compute its Picard group, at least for $h \gg g$. We start by describing explicitly uniform cyclic covers.

REMARK 4.3. Let $\mathcal{Y}_{n}$ be the stack parametrizing pairs $(\mathcal{L}, s)$ where $\mathcal{L}$ is an invertible sheaf and $s \in \mathcal{L}^{n}$. There is an equivalence $\mathcal{Y}_{n} \longrightarrow \mathcal{U C}_{n}$ that maps $(\mathcal{L}, s) \in \mathcal{Y}_{n}(S)$ to

$$
X=\operatorname{Spec} \mathscr{A} \longrightarrow S \quad \text { where } \quad \mathscr{A}=\mathcal{O}_{S} \oplus \mathcal{L}^{-1} \oplus \cdots \oplus \mathcal{L}^{-(n-1)},
$$

where $\mu_{n}$ acts on $\mathscr{A}$ via the given grading and the equivariant algebra structure on $\mathscr{A}$ is obtained as follows: given $0 \leqslant u, v, z<n$ such that $z \equiv u+n \bmod (n)$, the multiplication is

$$
\left(\mathcal{L}^{-u} \otimes \mathcal{L}^{-v} \longrightarrow \mathcal{L}^{-z}\right) \simeq \begin{cases}\mathcal{L}^{-u-v} \stackrel{\mathrm{id}}{\longrightarrow} \mathcal{L}^{-z} & \text { if } u+v<n \\ \mathcal{L}^{-u-v} \simeq \mathcal{L}^{-z} \otimes \mathcal{L}^{-n} \stackrel{\mathrm{id} \otimes s}{\longrightarrow} \mathcal{L}^{-z} & \text { if } u+v \geqslant n\end{cases}
$$

A quasi-inverse $\Lambda: \mathcal{U C}_{n} \longrightarrow \mathcal{Y}_{n}$ is obtained as follows. Given a uniform cyclic cover $f: X \longrightarrow S$ of degree $n$, the group $\mu_{n}$ acts on $f_{*} \mathcal{O}_{X}$. The degree one part of $f_{*} \mathcal{O}_{X}$ is an invertible sheaf on $S$ and we set $\mathcal{L}$ for its dual. Since the multiplication $f_{*} \mathcal{O}_{X} \otimes f_{*} \mathcal{O}_{X} \longrightarrow f_{*} \mathcal{O}_{X}$ is $\mu_{n}$-equivariant, we get a map from $\mathcal{L}^{-n}$ to the degree zero part of $f_{*} \mathcal{O}_{X}$, which is $\mathcal{O}_{S}$. This yields a section $s \in \mathcal{L}^{n}$.

Proposition 4.4. Let $h$ be a natural number and set $d=d(h, g, n)$. If $d \notin \mathbb{N}$, then $\mathcal{B}_{h, g, n}=\emptyset$. If $d \in \mathbb{N}$, the functor (see Remark 4.3 for the notation)

$$
\begin{gathered}
\mathcal{B}_{h, g, n} \stackrel{\psi_{h, g, n}}{\longrightarrow} \mathcal{U}_{d, g, n} \\
(D, C, f) \longmapsto\left(C, \Lambda_{C}(f)\right)
\end{gathered}
$$

is well defined and an open immersion. If $h>n(g-1)+1$, the image of $\psi_{h, g, n}$ is the complement of $\mathcal{Z}_{d, g, n}$ in $\mathcal{U}_{d, g, n}$, which is the étale locus of the canonical cover $\mathcal{H}_{d, g, n} \longrightarrow \mathcal{U}_{d, g, n}$. If $h=n(g-1)+1$ and $g \geqslant 1$, the image of $\psi_{h, g, n}$ is the substack of $\mathcal{U}_{0, g, n}$ of triples $(C \longrightarrow S, \mathcal{Q}, s)$ such that $\mathcal{Q}, \ldots, \mathcal{Q}^{n-1}$ are not trivial on the geometric fibers of $C \longrightarrow S$.

Proof. Let $(D, C, f) \in \mathcal{B}_{h, g, n}(k)$, where $k$ is an algebraically closed field, and set $(\mathcal{Q}, s)=\Lambda_{C}(f)$, so that

$$
f_{*} \mathcal{O}_{D} \simeq \mathcal{O}_{C} \oplus \mathcal{Q}^{-1} \oplus \cdots \oplus \mathcal{Q}^{-(n-1)}
$$

Since $D$ is integral and connected, we get $\operatorname{dim}_{k} \mathrm{H}^{0}\left(\mathcal{Q}^{-i}\right)=0$ for $i=1, \ldots, n-1$. By RiemannRoch it follows that $\operatorname{dim}_{k} \mathrm{H}^{1}\left(\mathcal{Q}^{-i}\right)=i \operatorname{deg} \mathcal{Q}+g-1$ and therefore

$$
\operatorname{dim}_{k} \mathrm{H}^{1}\left(f_{*} \mathcal{O}_{D}\right)=h=n(g-1)+1+(\operatorname{deg} \mathcal{Q}) n(n-1) / 2
$$

In particular, $\operatorname{deg} \mathcal{Q}=d \in \mathbb{Z}$. On the other hand, $s$ cannot be zero since $D$ is smooth and therefore $\operatorname{deg} \mathcal{Q} \geqslant 0$. In conclusion, we see that $\mathcal{B}_{h, g, n}=\emptyset$ if $d \notin \mathbb{N}$, and that $\psi_{h, g, n}$ is well defined if $d \in \mathbb{N}$.

From now on we assume $d \in \mathbb{N}$. From Remark 4.3 it follows that $\psi_{h, g, n}$ is fully faithful. 


\section{Flavia Poma, Mattia Talpo and Fabio Tonini}

Given $(C \longrightarrow S, \mathcal{Q}, s) \in \mathcal{U}_{d, g, n}$, we have to check under what conditions the total space of the uniform cyclic cover $D \longrightarrow C$ associated with $(\mathcal{Q}, s)$ (see Remark 4.3) is a smooth curve of genus $h$ over $S$. It is easy to see that everything follows from the case $S=\operatorname{Spec} k$, where $k$ is an algebraically closed field. Assume $d>0$. We have $\mathrm{H}^{0}\left(\mathcal{Q}^{-i}\right)=0$ for $i>0$, which tells us that $D$ is connected and, by definition of $d$, that $\operatorname{dim}_{k} \mathrm{H}^{1}\left(\mathcal{O}_{D}\right)=h$. The result then follows because the scheme $D$ is regular if and only if the zero locus of $s \in \mathcal{Q}^{n}$ is étale over $k$. This can be checked locally using that if $\left(R, m_{R}\right)$ is a DVR and $h \in R$, then $R[x] /\left(x^{n}-h\right)$ is regular if and only if $h \notin m_{R}^{2}$.

Now assume $d=0$. By Proposition 3.5 the map $s: \mathcal{O}_{C} \longrightarrow \mathcal{Q}^{n}$ is an isomorphism. In particular, $D \longrightarrow C$ is a $\mu_{n}$-torsor and therefore $D$ is smooth. Moreover, $D$ is connected if and only if $\mathrm{H}^{0}\left(\mathcal{Q}^{-i}\right)=0$ for $i=1, \ldots, n-1$, in which case it has exactly genus $h$ by definition of $d$. Since $\mathcal{Q}$ has degree zero, we have $\mathrm{H}^{0}\left(\mathcal{Q}^{-i}\right) \neq 0$ if and only if $\mathcal{Q}^{i} \simeq \mathcal{O}_{C}$, which concludes the proof.

Proposition 4.5. Let $h$ be a natural number with $d=d(h, g, n) \in \mathbb{N}$. Then $\mathcal{B}_{h, g, n}$ is a nonempty algebraic stack of finite type and if $n d>2 g-2$ or $p \nmid n, \mathcal{B}_{h, g, n} \longrightarrow \mathcal{M}_{g}$ is smooth and surjective.

Proof. Let $k$ be an algebraically closed field. We are going to prove that $\mathcal{B}_{h, g, n} \neq \emptyset$ and, if $d n>2 g-2$ or $p \nmid n$, that $\mathcal{B}_{h, g, n} \longrightarrow \mathcal{M}_{g}$ is surjective. All the other claims follow from Remark 3.3 and Propositions 3.7 and 4.4

Assume $d>0$. By Proposition 4.4 we have $\mathcal{B}_{h, g, n}=\mathcal{U}_{d, g, n}-\mathcal{Z}_{d, g, n}$. Moreover, by Proposition 3.7 there is a surjective map $\mathcal{U}_{d, g, n}-\mathcal{Z}_{d, g, n} \longrightarrow \mathcal{U}_{n d, g, 1}-\mathcal{Z}_{n d, g, 1}$. We can conclude that $\mathcal{B}_{h, g, n} \longrightarrow \mathcal{M}_{g}$ is surjective because if $C$ is a genus $g$ curve over $k$ and $p_{1}, \ldots, p_{n d}$ are distinct rational points, then $\left(C, \mathcal{O}_{C}\left(p_{1}+\cdots+p_{n d}\right), 1\right) \in\left(\mathcal{U}_{n d, g, 1}-\mathcal{Z}_{n d, g, 1}\right)(k)$.

Assume $d=0$ and let $C$ be a genus $g$ curve over $k$. We have $g \geqslant 1$ because if $g=0$, then $h=1-n<0$. By Proposition 4.4 the fiber of $\mathcal{B}_{h, g, n} \longrightarrow \mathcal{M}_{g}$ over $C \in \mathcal{M}_{g}(k)$ is not empty if and only if Pic $C$ has an element of order $n$. If $p \nmid n$, this is always the case thanks to Remark 1.12. If $p \mid n$ we have to show that this holds when $C$ is general. We may assume $n=q^{l}$ for some prime $q$. If $q \neq p$, then Pic $C$ has an element of order $n$ by Remark 1.12. Assume $p=q$. By [FvdG04, Theorem 2.3] when $C$ is general there exists an invertible sheaf $\mathcal{T}$ on $\mathcal{C}$ of order $p$. Since $\left[p^{l-1}\right]: \underline{\mathrm{Pic}}_{C}^{0} \longrightarrow \underline{\mathrm{Pic}}_{C}^{0}$ is surjective by Remark 1.12, there exists $\mathcal{Q} \in \operatorname{Pic} C$ such that $\mathcal{Q}^{p^{l-1}} \simeq \mathcal{T}$. It is easy to check that $\mathcal{Q}$ has order exactly $p^{l}=n$.

The following result explains the relation between Pic $\mathcal{B}_{h, g, n}$ and $\operatorname{Pic} \mathcal{J} a c_{d(h, g, n), g}$.

Proposition 4.6. Let $h$ be a natural number such that $d=d(h, g, n) \in \mathbb{Z}$ and $n d>2 g-2$. Let $\pi: \mathcal{C} \longrightarrow \mathcal{J} a c_{d, g}$ be the universal curve and let $\mathcal{L}$ be the universal invertible sheaf on $\mathcal{C}$. Set also $\mathcal{T}=\left(\operatorname{det} \pi_{*}\left(\mathcal{L}^{n} \otimes \omega_{\pi}\right)\right)^{2} \otimes\left(\operatorname{det} \pi_{*} \omega_{\pi}\right)^{-2}$. Then the map $\mathcal{B}_{h, g, n} \longrightarrow \mathcal{J} a c_{d, g}$ induces a surjective morphism

$$
\gamma:\left(\operatorname{Pic} \mathcal{J} a c_{d, g}\right) /\langle\mathcal{T}\rangle \longrightarrow \operatorname{Pic} \mathcal{B}_{h, g, n}
$$

If $\mathcal{Z}_{d, g, n}$ is integral, then $\gamma$ is an isomorphism. If $h=n=2, g=1$ (so that $d=1$ ) and $p \neq 2$, then the kernel of $\gamma$ is generated by $\left(\pi_{*} \mathcal{L}\right)^{2} \otimes\left(\pi_{*} \omega_{\pi}\right)^{-2}$.

If $g=0$, with notations from Proposition 2.6, then $\mathcal{T} \simeq \mathcal{L}_{0}^{2 n(n d-1)}$ if $d$ is even, $\mathcal{T} \simeq \mathcal{L}_{0}^{n(n d-1)}$ if $d$ is odd. If $g=1$ and $p \nmid d$, then $\mathcal{T} \simeq\left(\operatorname{det} \pi_{*} \mathcal{L}\right)^{2 n^{2}} \otimes\left(\pi_{*} \omega_{\pi}\right)^{n(d n+d-2 n)}$. If $g \geqslant 2$ and $p=0$, then $\mathcal{T} \simeq\left(\operatorname{det} \pi_{*} \omega_{\pi}\right)^{-2 n^{2}} \otimes d_{\pi}(\mathcal{L})^{n(n-1)} \otimes\left(\operatorname{det} \pi_{*}\left(\mathcal{L} \otimes \omega_{\pi}\right)\right)^{n(n+1)}$.

Proof. Notice that we must have $d \geqslant 1$, otherwise $g=0, d=0$ and $h=1-n \leqslant-1$. The stack $\mathcal{J} a c_{d, g}$ is smooth and irreducible by Remark 2.2. Moreover, $\mathcal{V}_{d, g, n} \longrightarrow \mathcal{J}_{a c_{d, g}}$ is a vector bundle of rank $n d+1-g$ and $\mathcal{U}_{d, g, n}$ is the complement of the zero section in $\mathcal{V}_{d, g, n}$ by Remark 3.3. 


\section{StACKS OF UNIFORM CYCLIC COVERS OF CURVES AND THEIR PICARD GROUPS}

Notice that $\operatorname{rk} \mathcal{V}_{d, g, n}=n d+1-g \geqslant 2$ using that $n d \geqslant \max \{2,2 g-1\}$. Thus $\mathcal{U}_{d, g, n}$ is smooth and integral and, by Proposition 1.9, we can conclude that the map $\mathcal{U}_{d, g, n} \longrightarrow \mathcal{J} a c_{d, g}$ induces an isomorphism on Picard groups. By Propositions 4.4 and 4.5 we have $\mathcal{B}_{h, g, n}=\mathcal{U}_{d, g, n}-\mathcal{Z}_{d, g, n} \neq \emptyset$, while by Proposition 1.9 and Theorem 3.2 the map $\gamma$ is well defined, surjective and, if $\mathcal{Z}_{d, g, n}$ is integral, an isomorphism. The claim about the case $h=n=2, g=1$ and $p \neq 2$ follows again by Proposition 1.9 and Theorem 3.2.

The expressions for $\mathcal{T}$ in the last part of the statement follow by a direct computation from Proposition 2.6 and Theorems 2.9 and 2.5, respectively.

Proof of Theorem A, except the case of $\mathcal{B}_{1,1,2}$. The first part of the statement follows from Propositions 4.4 and 4.5 .

By construction $\mathcal{L}$ is the universal invertible sheaf on $\mathcal{C}$ with respect to the map $\mathcal{B}_{h, g, n} \longrightarrow$ $\mathcal{J} a c_{d, g}$. We first consider the case $g=1$ and $h=n=2$. By Theorem 2.9 and Proposition 4.6 the group Pic $\mathcal{B}_{2,1,2}$ is generated by $\alpha=\pi_{*} \mathcal{L}$ and $\beta=\pi_{*} \omega_{\pi}$ with relations $8 \alpha=2 \beta, 2 \alpha=2 \beta$ and $12 \beta=0$. Those relations are equivalent to $6 \beta=0$ and $2 \alpha=2 \beta$, which yields Pic $\mathcal{B}_{2,1,2} \simeq$ $\mathbb{Z} / 6 \mathbb{Z} \times \mathbb{Z} / 2 \mathbb{Z}$.

In all the other cases we have $n d>2 g-2$ and that $\mathcal{Z}_{d, g, n}$ is integral by Theorem 3.2. In particular, the map $\gamma$ defined in Proposition 4.6 is an isomorphism. Using Proposition 4.6 the description of Pic $\mathcal{B}_{h, g, n}$ with generators and relations follows from Proposition 2.6 for $g=0$, Theorem 2.9 for $g=1$ and from Theorem 2.5 for $g \geqslant 2$.

We now deal with the description as abstract groups. For $g=0$ the result is clear.

Consider now the case (ii), that is, $g=1$ and $n d>2$. Set $A=n(d n+d-2 n)$ and notice that $2 \mid A$. The group $H=\operatorname{Pic} \mathcal{B}_{h, 1}$ is isomorphic to $\mathbb{Z}^{2} /\left\langle(0,12),\left(2 n^{2}, A\right)\right\rangle$. The element $(0,4)$ has order three in $H$. A direct check shows that the map $\phi: H \longrightarrow \mathbb{Z} / 3 \mathbb{Z}$ given by $\phi(0,1)=1$ and $\phi(1,0)=x$, where $x=0$ if $3 \mid n$ and $x=A / n^{2}$ otherwise, is well defined because $3 \mid n$ implies $3 \mid A$. Since $\phi(0,4)=1$ we obtain $H \simeq \mathbb{Z} / 3 \mathbb{Z} \times G$, where $G=H /\langle(0,4)\rangle$. We have $G=\mathbb{Z}^{2} /\left\langle(0,4),\left(2 n^{2}, A\right)\right\rangle$. If $4 \mid A$, then $G \simeq \mathbb{Z} / 4 \mathbb{Z} \times \mathbb{Z} / 2 n^{2} \mathbb{Z}$. So assume $A \equiv 2 \bmod (4)$. The map $\psi: G \longrightarrow \mathbb{Z} / 2 \mathbb{Z}$ given by $\psi(u, v)=v$ is well defined. Moreover, $\left(n^{2}, 1\right)$ has order two in $G$ and $\psi\left(n^{2}, 1\right)=1$. We obtain $G=\mathbb{Z} / 2 \mathbb{Z} \times \mathbb{Z}^{2} /\left\langle(0,4),\left(n^{2}, 1\right)\right\rangle$. It is now easy to check that the last factor is cyclic of order $4 n^{2}$.

Consider now the case (iii) and set $x=(1,0,0) \in \mathbb{Z}^{3}$. Then Pic $\mathcal{B}_{h, g, n}$ is isomorphic to the group $H$ quotient of $\mathbb{Z}^{3}$ by the relations $\left(-2 n^{2}, n(n+1), n(n-1)\right)$ and, if $g=2,10 x$. Set $l=10$ if $g=2$ and $l=0$ otherwise. It is easy to see that $\langle x\rangle \simeq \mathbb{Z} / l \mathbb{Z}$. A direct computation shows that the map $\psi: H \longrightarrow \mathbb{Z} / l \mathbb{Z}$ given by $\psi(u, v, z)=u+v+z$ is well defined. Since $\psi(x)=1$, we can conclude that $H \simeq \mathbb{Z} / l \mathbb{Z} \times G$, where $G=H / x \simeq \mathbb{Z}^{2} /\langle(n(n+1), n(n-1))\rangle$. Set $m$ for the great common divisor of $n(n+1)$ and $n(n-1)$. An easy computation shows that $m=n$ if $n$ is even and $m=2 n$ is $n$ is odd. Let $\alpha, \beta \in \mathbb{Z}$ such that $\alpha n(n+1)+\beta n(n-1)=m$. Consider the map

$$
\phi=\left(\begin{array}{cc}
\alpha & \beta \\
-\frac{n(n-1)}{m} & \frac{n(n+1)}{m}
\end{array}\right): \mathbb{Z}^{2} \longrightarrow \mathbb{Z}^{2} .
$$

By construction $\phi$ is an isomorphism because $\operatorname{det} \phi=1$. Moreover, $\phi(n(n+1), n(n-1))=(m, 0)$ and therefore $G \simeq \mathbb{Z}^{2} /\langle(m, 0)\rangle \simeq \mathbb{Z} / m \mathbb{Z} \times \mathbb{Z}$ as required.

In the remaining part of this section we will deal with the case of $\mathcal{B}_{1,1,2}$. As pointed out at the beginning, this case is peculiar and needs a variation of the methods used for higher genera. Nevertheless, the steps in the computation of Pic $\mathcal{B}_{1,1,2}$ are very similar to the ones in the computation of $\operatorname{Pic} \mathcal{B}_{h, 1, n}$, for $h \gg 0$. 


\section{Flavia Poma, Mattia Talpo and Fabio Tonini}

In what follows we consider $g=1$ and assume $p \nmid 6$. We denote by $\widetilde{\mathcal{M}}_{1,2}$ the universal curve over $\mathcal{M}_{1,1}$, which is the moduli stack of triples $\left(E, \sigma_{1}, \sigma_{2}\right)$ where $E$ is a genus one curve and $\sigma_{1}$, $\sigma_{2}$ are sections. The map $\widetilde{\mathcal{M}}_{1,2} \longrightarrow \mathcal{M}_{1,1}$ is the functor that forgets the second section.

Proposition 4.7. The functor

$$
\begin{gathered}
\widetilde{\mathcal{M}}_{1,2} \longrightarrow \mathcal{J} a c_{0,1} \\
\left(E, \sigma_{1}, \sigma_{2}\right) \longmapsto\left(E, \mathcal{O}_{E}\left(\sigma_{2}-\sigma_{1}\right)\right)
\end{gathered}
$$

is an epimorphism in the fppf topology and is a section of the functor

$$
\begin{aligned}
& \mathcal{J} a c_{0,1} \longrightarrow \widetilde{\mathcal{M}}_{1,2} \\
& (E, \mathcal{Q}) \longmapsto\left(\underline{\operatorname{Pic}}_{E / S}^{0},\left[\mathcal{O}_{E}\right],[\mathcal{Q}]\right)
\end{aligned}
$$

Proof. The second part of the statement follows from Lemma 2.11. For the first one let $(E, \mathcal{Q}) \in$ $\mathcal{J} a c_{0,1}$. We may assume that $E$ has a section $\sigma_{1}$. Again by Lemma 2.11, $[\mathcal{Q}]=\left[\mathcal{O}_{E}\left(\sigma_{2}-\sigma_{1}\right)\right]$, for some section $\sigma_{2}$ of $E$, which means that $\mathcal{Q}$ and $\mathcal{O}_{E}\left(\sigma_{2}-\sigma_{1}\right)$ are fppf locally isomorphic.

Definition 4.8. We define the group functor $G_{0}$ over $\widetilde{\mathcal{M}}_{1,2}$ as the group $G$ obtained as in Lemma 2.14 with respect to the maps defined in Proposition 4.7.

Proposition 4.9. An element of $G_{0}\left(S \stackrel{\left(E, \sigma_{1}, \sigma_{2}\right)}{\longrightarrow} \widetilde{\mathcal{M}}_{1,2}\right)$ is a pair $(f, \lambda)$ where $f:\left(E, \sigma_{1}\right) \longrightarrow$ $\left(E, \sigma_{1}\right)$ is a translation and $\lambda: \mathcal{O}_{E}\left(\sigma_{2}-\sigma_{1}\right) \longrightarrow \mathcal{O}_{E}\left(f\left(\sigma_{2}\right)-f\left(\sigma_{1}\right)\right)$ is an isomorphism. Moreover, we have an exact sequence

$$
\begin{gathered}
(f, \lambda) \longmapsto f\left(\sigma_{1}\right) \\
0 \longrightarrow \mathbb{G}_{m} \longrightarrow 0 \\
\mu \longmapsto(\mathrm{id}, \mu)
\end{gathered}
$$

in the Zariski topology of $\mathrm{Sch} / \widetilde{\mathcal{M}}_{1,2}$, where $\mathcal{E} \longrightarrow \widetilde{\mathcal{M}}_{1,2}$ is the universal curve. In particular, $G_{0}$ is smooth over $\widetilde{\mathcal{M}}_{1,2}$.

Proof. By definition, an element of $G_{0}\left(S \stackrel{\left(E, \sigma_{1}, \sigma_{2}\right)}{\longrightarrow} \widetilde{\mathcal{M}}_{1,2}\right)$ is a pair $(f, \lambda)$ where $f: E \longrightarrow E$ is an isomorphism such that $f_{*}: \underline{\operatorname{Pic}}_{E / S}^{0} \longrightarrow \underline{\operatorname{Pic}}_{E / S}^{0}$ is the identity and

$$
\lambda: \mathcal{O}_{E}\left(\sigma_{2}-\sigma_{1}\right) \longrightarrow \mathcal{O}_{E}\left(f\left(\sigma_{2}\right)-f\left(\sigma_{1}\right)\right)
$$

is an isomorphism. As in the proof of Proposition 2.16, $f_{*}=\mathrm{id}$ means that $f$ is a translation. In particular the sequence in the statement is well defined and, since $\pi_{*} \mathbb{G}_{m, E} \simeq \mathbb{G}_{m}$, it is exact in the first two terms.

It remains to prove that $G_{0} \longrightarrow \mathcal{E}$ is a Zariski epimorphism. This will also imply that $G_{0}$ is locally a product of $\mathbb{G}_{m}$ and $\mathcal{E}$ and therefore smooth. Let $\left(E, \sigma_{1}, \sigma_{2}, \delta\right) \in \mathcal{E}$ and let $t$ be the translation by $\delta$, so that $t\left(\sigma_{1}\right)=\delta$. Since $t$ is a translation, we have

$$
\left[\mathcal{O}_{E}\left(\sigma_{2}-\sigma_{1}\right)\right]=\left[\mathcal{O}_{E}\left(t\left(\sigma_{2}\right)-t\left(\sigma_{1}\right)\right)\right] \text { in } \underline{\operatorname{Pic}}_{E / S}^{0},
$$

which means that the sheaves differ from an invertible sheaf coming from the base thanks to Lemma 1.10. So Zariski locally we get an isomorphism $\lambda: \mathcal{O}_{E}\left(\sigma_{2}-\sigma_{1}\right) \longrightarrow \mathcal{O}_{E}\left(t\left(\sigma_{2}\right)-t\left(\sigma_{1}\right)\right)$ and therefore $\left.(t, \lambda) \in G_{0}\left(E, \sigma_{1} . \sigma_{2}\right)\right)$ is mapped to $\left(E, \sigma_{1}, \sigma_{2}, \delta\right)$. 


\section{StACKS OF UNIFORM CYCLIC COVERS OF CURVES AND THEIR PICARD GROUPS}

LEMmA 4.10. Let $\mathcal{X}$ be a smooth algebraic stack, let $\mathcal{Y} \stackrel{p}{\longrightarrow} \mathcal{X}$ be a $\mathbb{G}_{m}$-torsor and let $\mathcal{L}$ be the invertible sheaf over $\mathcal{X}$ corresponding to it. Then we have an exact sequence

$$
\mathbb{Z} \stackrel{\mathcal{L}}{\longrightarrow} \text { Pic } \mathcal{X} \stackrel{p^{*}}{\longrightarrow} \text { Pic } \mathcal{Y} \longrightarrow 0 \text {. }
$$

Proof. The stack $\mathcal{Y}$ and the line bundle $\mathcal{Y}^{\prime} \longrightarrow \mathcal{X}$ corresponding to $\mathcal{L}$ can be seen as the relative sheaves

$$
\underline{\mathrm{IsO}}_{\mathcal{X}}\left(\mathcal{O}_{\mathcal{X}}, \mathcal{L}\right) \text { and } \underline{\operatorname{Hom}}_{\mathcal{X}}\left(\mathcal{O}_{\mathcal{X}}, \mathcal{L}\right)
$$

on $\operatorname{Sch} / \mathcal{X}$, respectively. Denote by $q: \mathcal{Y}^{\prime} \longrightarrow \mathcal{X}$ the structural morphism. The stack $\mathcal{Y}$ is the open substack of $\mathcal{Y}^{\prime}$ whose complement $\mathcal{Z}$ is the zero section of $\mathcal{Y}^{\prime} \longrightarrow \mathcal{X}$. The stack $\mathcal{Z}$ is integral since $\mathcal{X}$ is so and it is the zero locus of the universal section of $q^{*} \mathcal{L}$. The result then follows from Proposition 1.9.

In what follows we denote by $\mathcal{F}$ the complement of the zero section $\mathcal{M}_{1,1} \longrightarrow \widetilde{\mathcal{M}}_{1,2}$ in $\widetilde{\mathcal{M}}_{1,2}[2]$.

Proposition 4.11. The composition $\mathcal{B}_{1,1,2} \longrightarrow \mathcal{J} a c_{0,1} \longrightarrow \widetilde{\mathcal{M}}_{1,2}$ has image in $\mathcal{F}$. The induced map $\mathcal{B}_{1,1,2} \longrightarrow \mathcal{F}$ yields an isomorphism on Picard groups and factors as a $\mathbb{G}_{m}$-torsor $\mathcal{B}_{1,1,2} \longrightarrow$ $\mathrm{B}_{\mathcal{F}} G_{0}$ followed by the projection $\mathrm{B}_{\mathcal{F}} G_{0} \longrightarrow \mathcal{F}$.

Proof. Set $\mathcal{X}=\mathrm{B}_{\mathcal{F}} G_{0}$. By Lemma 2.14 and Proposition 4.7, we see that $\mathcal{J} a c_{0,1} \simeq \mathrm{B}_{\widetilde{\mathcal{M}}_{1,2}} G_{0}$. In particular, $\mathcal{X}$ can be viewed as the closed substack of $\mathcal{J} a c_{0,1}$ of pairs $(E, \mathcal{Q})$ such that $\left(\underline{\operatorname{Pic}}_{E}^{0},\left[\mathcal{O}_{E}\right],[\mathcal{Q}]\right) \in \mathcal{F}$. By Proposition 4.4 we see that the forgetful map $\mathcal{B}_{1,1,2} \longrightarrow \mathcal{X}$ is a $\mathbb{G}_{m}$-torsor corresponding to the invertible sheaf $\pi_{*}\left(\mathcal{L}^{2}\right)$, where $\pi: \mathcal{E} \longrightarrow \mathcal{X}$ is the universal curve and $\mathcal{L}$ is the universal invertible sheaf over it. Notice that $\mathcal{X}$ is smooth because it is an open substack of $\mathcal{J} a c_{0,1}$, which is smooth thanks to Remark 2.2. In particular, from Lemma 4.10 the pull-back of $\mathcal{B}_{1,1,2} \longrightarrow \mathcal{X}$ induces an isomorphism

$$
\operatorname{Pic} \mathcal{B}_{1,1,2} \simeq \operatorname{Pic} \mathcal{X} /\left\langle\pi_{*}\left(\mathcal{L}^{2}\right)\right\rangle \text {. }
$$

Moreover, from Remark 1.2, we have $\operatorname{Pic} \mathcal{X}=\operatorname{Pic} \mathcal{F} \oplus G_{0}^{\vee}$. We are going to show that $G_{0}^{\vee} \simeq \mathbb{Z}$ and that the component of $\pi_{*}\left(\mathcal{L}^{2}\right)$ in Pic $\mathcal{X}$ with respect to $G_{0}^{\vee}$ generates this last group. This will imply that the composition of pull-backs $\operatorname{Pic} \mathcal{F} \longrightarrow \operatorname{Pic} \mathcal{X} \longrightarrow$ Pic $\mathcal{B}_{1,1,2}$ is an isomorphism.

Taking into account Proposition 4.9, the inclusion $\mathbb{G}_{m} \longrightarrow G_{0}$ gives a map $\alpha: G_{0}^{\vee} \longrightarrow \mathbb{G}_{m}^{\vee} \simeq \mathbb{Z}$ whose kernel is the group of characters of the universal curve $\widetilde{\mathcal{E}}$ of $\mathcal{F}$. If $\phi: \widetilde{\mathcal{E}} \longrightarrow \mathbb{G}_{m}$ is such a homomorphism, $\phi^{-1}(1)$ is a closed substack of $\widetilde{\mathcal{E}}$; by checking on the geometric fibers, we see that they are topologically equal. Since $\widetilde{\mathcal{E}}$ is reduced, we can conclude that $\phi$ is trivial and therefore that $\alpha$ is injective. As in the proof of Proposition 2.20, considering the functor

$$
\begin{gathered}
\mathrm{B}_{\mathcal{F}} \mathbb{G}_{m} \longrightarrow \mathcal{X} \\
\left(E \stackrel{\pi}{\longrightarrow} S, \sigma_{1}, \sigma_{2}, \mathcal{Q}\right) \longmapsto\left(E, \mathcal{O}_{E}\left(\sigma_{2}-\sigma_{1}\right) \otimes \pi^{*} \mathcal{Q}\right)
\end{gathered}
$$

and the expression

$$
\pi_{*}\left[\left(\mathcal{O}_{E}\left(\sigma_{2}-\sigma_{1}\right) \otimes \pi^{*} \mathcal{Q}\right)^{2}\right] \simeq \pi_{*}\left(\mathcal{O}_{E}\left(2 \sigma_{2}-2 \sigma_{1}\right)\right) \otimes \mathcal{Q}^{2},
$$

we see that $\pi_{*}\left(\mathcal{L}^{2}\right)$ is sent to 2 by the map

$$
\operatorname{Pic} \mathcal{X} \simeq \operatorname{Pic} \mathcal{F} \oplus G_{0}^{\vee} \stackrel{\operatorname{id} \oplus \alpha}{\longrightarrow} \operatorname{Pic} \mathcal{F} \oplus \mathbb{G}_{m}^{\vee} \longrightarrow \mathbb{G}_{m}^{\vee}=\mathbb{Z} .
$$

In particular, $2 \mathbb{Z} \subseteq \operatorname{Im} \alpha$ and we need to prove that those groups are equal, or, equivalently, that $\alpha$ is not an isomorphism. Assume by contradiction that $\alpha$ is an isomorphism. This exactly 


\section{Flavia Poma, Mattia Talpo and Fabio Tonini}

means that the map $\mathbb{G}_{m} \longrightarrow G_{0}$ has a section. Thus also the map $G_{0} \longrightarrow \widetilde{\mathcal{E}}$ has a section. Since this last map is a $\mathbb{G}_{m}$-torsor, we can rephrase this by saying that the invertible sheaf over $\widetilde{\mathcal{E}}$ corresponding to $G_{0}$ is trivial. We are going to compute this sheaf and prove that it is not trivial. Given $\left(E \stackrel{\pi}{\longrightarrow} S, \sigma_{1}, \sigma_{2}, \sigma_{3}\right) \in \widetilde{\mathcal{E}}$ and denoting by $t:\left(E, \sigma_{1}\right) \longrightarrow\left(E, \sigma_{1}\right)$ the translation by $\sigma_{3}$, so that $t\left(\sigma_{1}\right)=\sigma_{3}$, the invertible sheaf $\mathcal{K}$ over $\widetilde{\mathcal{E}}$ corresponding to $G_{0}$ is given by the following calculation:

$$
\begin{aligned}
\underline{\operatorname{IsO}}_{E}\left(\mathcal{O}_{E}\left(\sigma_{2}-\sigma_{1}\right), \mathcal{O}_{E}\left(t\left(\sigma_{2}\right)-t\left(\sigma_{1}\right)\right)\right. & \simeq \underline{\operatorname{IsO}}_{E}\left(\mathcal{O}_{E}\left(\sigma_{2}+\sigma_{3}-\sigma_{1}\right), \mathcal{O}_{E}\left(t\left(\sigma_{2}\right)\right)\right) \\
& \simeq \underline{\operatorname{IsO}}_{S}\left(\pi_{*} \mathcal{O}_{E}\left(\sigma_{2}+\sigma_{3}-\sigma_{1}\right), \mathcal{O}_{S}\right) \simeq \pi_{*} \mathcal{O}_{E}\left(\sigma_{2}+\sigma_{3}-\sigma_{1}\right)^{\vee},
\end{aligned}
$$

where we have used that $\pi_{*} \mathcal{O}_{E}\left(t\left(\sigma_{2}\right)\right) \simeq \mathcal{O}_{S}$ by Lemma 1.11. Using Proposition 2.12 twice we also have

$$
\pi_{*} \mathcal{O}_{E}\left(\sigma_{2}+\sigma_{3}-\sigma_{1}\right)^{\vee} \simeq \sigma_{1}^{*} \mathcal{O}_{E}\left(\sigma_{2}\right) \otimes \sigma_{1}^{*} \mathcal{O}_{E}\left(\sigma_{3}\right) \otimes \sigma_{3}^{*} \mathcal{O}_{E}\left(-\sigma_{2}\right) \otimes \pi_{*} \omega_{\pi} .
$$

Given an elliptic curve $E$ over an algebraically closed field with origin $p_{1}$ and $p_{2} \in E[2]-\left\{p_{1}\right\}$, we consider the object $\chi=\left(E \times E \stackrel{\mathrm{pr}_{2}}{\longrightarrow} E, \sigma_{1}, \sigma_{2}, \Delta\right) \in \widetilde{\mathcal{E}}(E)$ where $\sigma_{i}=p_{i} \times \operatorname{id}_{E}: E \longrightarrow E \times E$ for $i=1,2$ and $\Delta: E \longrightarrow E \times E$ is the diagonal. Using the isomorphism above, the pull-back of $\mathcal{K}$ to $E$ is given by

$$
\sigma_{1}^{*} \mathcal{O}_{E \times E}\left(\sigma_{2}\right) \otimes \sigma_{1}^{*} \mathcal{O}_{E \times E}(\Delta) \otimes \Delta^{*} \mathcal{O}_{E \times E}\left(-\sigma_{2}\right) \otimes \operatorname{pr}_{2 *} \omega_{\mathrm{pr}_{2}} \simeq \mathcal{O}_{E}\left(p_{1}-p_{2}\right),
$$

which is not trivial.

Proposition 4.12. We have Pic $\mathcal{F} \simeq \mathbb{Z} / 4 \mathbb{Z}$, generated by the invertible sheaf $\pi_{*} \omega_{\pi}$, where $\pi: \mathcal{E} \longrightarrow \mathcal{F}$ is the universal curve over $\mathcal{F}$.

Proof. Let $k$ be the base field and set $U=\operatorname{Spec} R$, where $R=k[a, b]_{\Delta}$ with $\Delta=4 a^{3}+27 b^{2}$ and $\rho: \mathcal{F} \longrightarrow \mathcal{M}_{1,1}$ for the structure map, which is an étale degree three cover. Since char $k \nmid 6$, the map $U \longrightarrow \mathcal{M}_{1,1}$ given by the general Weierstrass curve

$$
W=\operatorname{Proj}(R[x, y, z] /(f)) \longrightarrow U \text { where } f=y^{2} z-x^{3}-a x z^{2}-b z^{3}
$$

is a $\mathbb{G}_{m}$-torsor corresponding to a generator $\mathcal{K}$ of Pic $\mathcal{M}_{1,1}$, either $p_{*} \omega_{p}$ or its dual, where $p: \widetilde{\mathcal{E}} \longrightarrow$ $\mathcal{M}_{1,1}$ is the universal curve. In particular, the base change $V=U \times_{\mathcal{M}_{1,1}} \mathcal{F} \longrightarrow \mathcal{F}$ is the $\mathbb{G}_{m^{-}}$ torsor corresponding to the pull-back $\rho^{*} \mathcal{K}$; it coincides with $W[2]$ minus the zero section. By [Sil86, Group law algorithm 2.3], 2-torsion points are obtained by modding out by $y$; therefore we get

$$
V \simeq \operatorname{Spec} k[a, b, x]_{\Delta} /\left(x^{3}+a x+b\right) .
$$

In particular, $V$ is an open subscheme of $\mathbb{A}^{2}=\operatorname{Spec} k[a, x]$ and therefore Pic $V=0$. We can conclude by Lemma 4.10 that Pic $\mathcal{F}$ is generated by $\omega=\pi_{*} \omega_{\pi}$. This is because if $\omega^{\prime}=p_{*} \omega_{p}$, then $\rho^{*} \omega^{\prime} \simeq \omega$.

We want to prove that the order $r$ of $\omega$ in Pic $\mathcal{F}$ is exactly four. Since $\omega^{\prime}$ has order 12 in Pic $\mathcal{M}_{1,1}$ by Theorem 2.10, we have

$$
\omega^{r} \simeq \rho^{*} \omega^{\prime r} \simeq \mathcal{O}_{\mathcal{F}} \Longrightarrow \rho_{*} \mathcal{O}_{\mathcal{F}} \simeq \omega^{\prime r} \otimes \rho_{*} \mathcal{O}_{\mathcal{F}} \stackrel{\operatorname{det}}{\Longrightarrow} \omega^{\prime 3 r} \simeq \mathcal{O}_{\mathcal{M}_{1,1}} \Longrightarrow 12|3 r \Longrightarrow 4| r .
$$

Consider now the invertible sheaf $\mathcal{T}$ on $\mathcal{F}$ given by

$$
\mathcal{T}:\left(E \stackrel{q}{\longrightarrow} S, \sigma_{1}, \sigma_{2}\right) \longmapsto \sigma_{2}^{*} \mathcal{O}_{E}\left(\sigma_{2}-\sigma_{1}\right) \otimes \sigma_{1}^{*} \mathcal{O}_{E}\left(\sigma_{1}-\sigma_{2}\right) .
$$

Since $\mathcal{O}_{E}\left(\sigma_{2}-\sigma_{1}\right)^{2} \simeq q^{*} q_{*}\left(\mathcal{O}_{E}\left(\sigma_{2}-\sigma_{1}\right)^{2}\right)$ by definition of $\mathcal{F}$ and Lemma 1.10, we see that $\mathcal{T}^{2} \simeq \mathcal{O}_{\mathcal{F}}$. On the other hand, since $\sigma_{1}$ and $\sigma_{2}$ are disjoint, we have $\sigma_{1}^{*} \mathcal{O}_{E}\left(\sigma_{2}\right) \simeq \sigma_{2}^{*} \mathcal{O}_{E}\left(\sigma_{1}\right) \simeq \mathcal{O}_{E}$ 


\section{StACKS OF UNIFORM CYCLIC COVERS OF CURVES AND THEIR PICARD GROUPS}

and therefore $\mathcal{T} \simeq \omega^{2}$ thanks to Proposition 2.12. In conclusion $\mathcal{O}_{\mathcal{F}} \simeq \mathcal{T}^{2} \simeq \omega^{4}$ and therefore $r=4$.

Proof of Theorem $A$, the case of $\mathcal{B}_{1,1,2}$. By Proposition $4.4 \mathcal{L}$ is a degree zero invertible sheaf on $\mathcal{C}$ which is never trivial on the geometric fibers of $\mathcal{C} \longrightarrow \mathcal{B}_{1,1,2}$. By Grauert we can conclude that $\pi_{*} \mathcal{L}=0$, so that $\operatorname{det} \pi_{*} \mathcal{L}$ is trivial. The result then follows from Propositions 4.11 and 4.12.

\section{ACKNOWLEDGEMENTS}

We would like to thank our advisor Angelo Vistoli for suggesting the problem and for sharing his ideas.

We also thank Michele Bolognesi for directing us to Mumford's papers about abelian varieties, along with Dajano Tossici, Matthieu Romagny, Nicola Pagani, Filippo Viviani and Margarida Melo for useful conversations.

Finally, we thank the referee for useful comments and corrections.

\section{REFERENCES}

AV04 A. Arsie and A. Vistoli, Stacks of cyclic covers of projective spaces. Compos. Math. 140 (2004), no. 3, 647-666. http://dx.doi.org/10.1112/S0010437X03000253

BV12 M. Bolognesi and A. Vistoli, Stacks of trigonal curves, Trans. Amer. Math. Soc. 364 (2012), 3365-3393. http://dx.doi.org/10.1090/S0002-9947-2012-05370-0

FO10 W. Fulton and M. Olsson, The Picard group of $M_{1,1}$, Algebra Number Theory 4 (2010), no. 1, 87-104.

FvdG04 C. Faber and G. van der Geer, Complete subvarieties of moduli spaces and the Prym map, J. Reine Angew. Math. 573 (2004). http://dx.doi.org/10.1515/crll.2004.056

Gro66 A. Grothendieck, Éléments de géométrie algébrique IV-3, Étude locale des schémas et des morphismes de schémas (Troisième partie), rédigés avec la collaboration de Jean Dieudonné, Publ. Math. Inst. Hautes Études Sci. 28 (1966).

Gro67 A. Grothendieck, Éléments de géométrie algébrique IV-4, Étude locale des schémas et des morphismes de schémas (Quatrième partie), rédigés avec la collaboration de Jean Dieudonné, Publ. Math. Inst. Hautes Études Sci. 32 (1967).

Kle80 S. L. Kleiman, Relative Duality for Quasi-Coherent Sheaves, Compos. Math. 41 (1980), 39-60.

MBL99 L. Moret-Bailly and G. Laumon, Champs algébriques, Ergebnisse der Mathematik und ihrer Grenzgebiete, 3. Folge, A Series of Modern Surveys in Mathematics vol. 39, Springer-Verlag, Berlin, 1999.

Mil08 J. S. Milne. Abelian Varieties, Online lecture notes, 2008, available at http://www. jmilne.org/ math/CourseNotes/av.html

Mum63 D. Mumford, Picard groups of moduli problems, Arithmetical Algebraic Geometry, (Proc. Conf. Purdue Univ., 1963), 33-81, Haper \& Row, New York, 1963.

Mum66 D. Mumford, On the equations defining abelian varieties. I, Invent. Math. 1 (1966), no. 4, 287354.

MV14 M. Melo and F. Viviani, The Picard group of the compactified universal Jacobian, Doc. Math. 19 (2014), 457-506.

Pag13 N. Pagani, Moduli of abelian covers of elliptic curves, arXiv:1303.2991.

Sil86 J. H. Silverman, The Arithmetic of Elliptic Curves, Graduate Texts in Mathematics, 106, Springer-Verlag, New York, 1986. 


\section{Flavia Poma, Mattia Talpo and Fabio Tonini}

SP14 The Stacks Project Authors, Stacks Project, version 2014, available at http://math.columbia. edu/algebraic_geometry/stacks-git.

Flavia Poma flavia.poma@sns.it

Sissa, via Bonomea 265, 34136 Trieste, Italy

Mattia Talpo talpuz@mpim-bonn.mpg.de

Max Planck Institute for Mathematics, Vivatsgasse 7, 53111 Bonn, Germany

Fabio Tonini tonini@mathematik.hu-berlin.de

Humboldt University of Berlin, Unter den Linden 6, 10099 Berlin, Germany 